COMPUTED TOMOGRAPHY IMAGE DENOISING BASED ON MULTI DOSE CT IMAGE FUSION AND EXTENDED SPARSE TECHNIQUES

by

\author{
Anuyogam Venkataraman \\ BE, Anna University, Chennai, India, 2010 \\ A thesis \\ presented to Ryerson University \\ in partial fulfillment of the \\ requirements for the degree of \\ Master of Applied Science \\ in the program of \\ Electrical and Computer Engineering
}

Toronto, Ontario, Canada, 2014

(C) (Anuyogam Venkataraman) 2014 
I hereby declare that I am the sole author of this thesis. This is a true copy of the thesis, including any required final revisions, as accepted by my examiners.

I authorize Ryerson University to lend this thesis or dissertation to other institutions or individuals for the purpose of scholarly research.

I further authorize Ryerson University to reproduce this thesis by photocopying or by other means, in total or in part, at the request of other institutions or individuals for the purpose of scholarly research.

I understand that my thesis may be made electronically available to the public. 


\title{
COMPUTED TOMOGRAPHY IMAGE DENOISING BASED ON MULTI DOSE CT IMAGE FUSION AND EXTENDED SPARSE TECHNIQUES
}

\author{
Master of Applied Science 2014 \\ Anuyogam Venkataraman \\ Electrical and Computer Engineering \\ Ryerson University
}

\begin{abstract}
With the increasing utilization of X-ray Computed Tomography (CT) in medical diagnosis, obtaining higher quality image with lower exposure to radiation is a highly challenging task in image processing. Sparse representation based image fusion is one of the sought after fusion techniques among the current researchers. A novel image fusion algorithm based on focused vector detection is proposed in this thesis. Firstly, the initial fused vector is acquired by combining common and innovative sparse components of multi-dosage ensemble using Joint Sparse PCA fusion method utilizing an overcomplete dictionary trained using high dose images of the same region of interest from different patients. And then, the strongly focused vector is obtained by determining the pixels of low dose and medium dose vectors which have high similarity with the pixels of the initial fused vector using certain quantitative metrics. Final fused image is obtained by denoising and simultaneously integrating the strongly focused vector, initial fused vector and source image vectors in joint sparse domain thereby preserving the edges and other critical information needed for diagnosis. This thesis demonstrates the effectiveness of the proposed algorithms when experimented on different images and the qualitative and quantitative results are compared with some of the widely used image fusion methods.
\end{abstract}




\section{Acknowledgements}

I would like to thank my supervisor Prof. Javad Alirezaie, for his outstanding support, encouragement and endless patience throughout the course of my research work. It was a great learning experience for me to complete this research under his valuable guidance. I would also like to extend my gratitude to Dr. Paul Babyn for providing helpful insights and needful resources without which this research work would not have been possible.

I would like to dedicate this thesis to my beloved husband who has stood by me during all my difficult times and has been a great mental and physical support throughout my graduate studies. I would not have been where I am today without his motivation and endurance. I also thank my parents and sister who have always wished only the best for me. I would like to thank all my CVIP lab friends for their timely help and especially Samira who has supported me with lots of optimism during the tougher times and for making this journey a memorable experience. 


\section{TABLE OF CONTENTS}

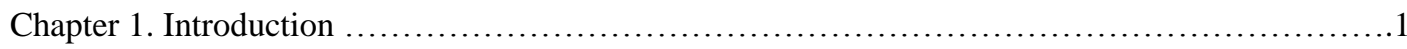

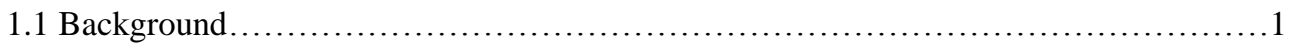

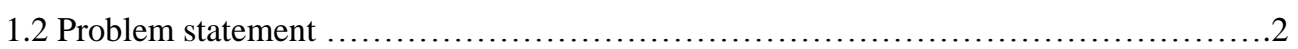

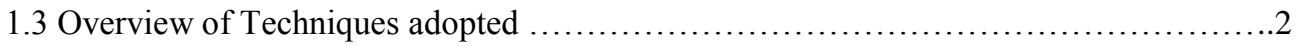

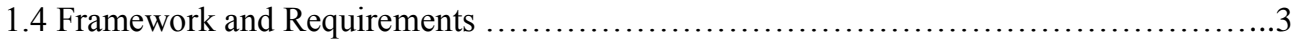

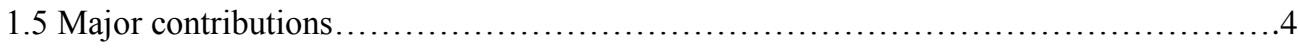

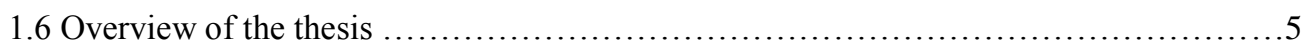

Chapter 2. Sparse Representation

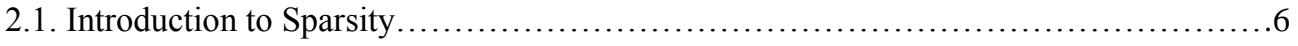

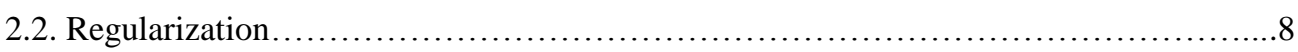

2.3. Spare Approximation........................................................... 9

2.4. Example of sparse representation and approximation.............................. 10

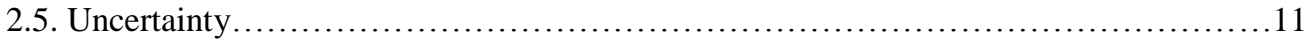

2.6. Uniqueness of sparse representation........................................... 12

2.7. Necessity of sparse approximation..................................................

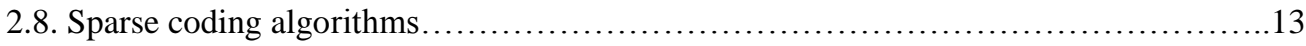

2.8.1. LASSO and Basis Pursuit................................................... 13

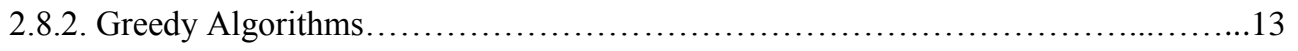

2.8.2.1. Matching Pursuit with Time-Frequency Dictionaries..........................14

2.8.2.2. Weak Matching Pursuit algorithm........................................... 15

2.8.2.3. Orthogonal Matching Pursuit algorithm..................................... 17

2.8.2.4. Stage wise Orthogonal Matching Pursuit...................................... 18

Chapter 3. Dictionaries

3.1. Comparison of analytical and adaptive dictionary.................................. 21

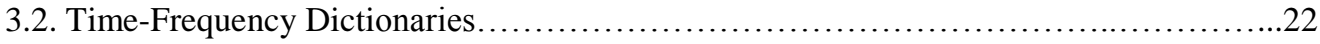

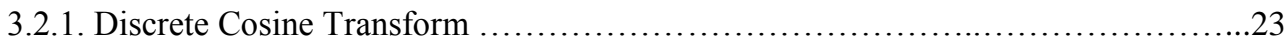

3.2.2. Overcomplete Discrete Cosine Transform........................................23

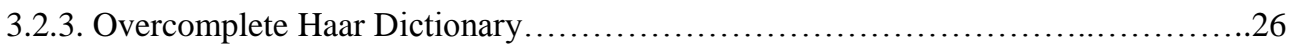


3.2.5. Continuous wavelet Transform with discrete wavelet coefficients...................27

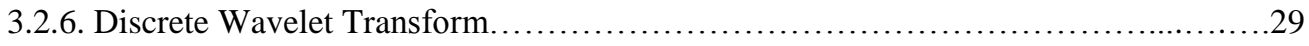

3.2.7. Dual Tree Complex Wavelet transform..................................... 30

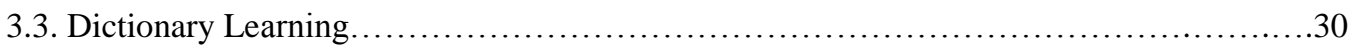

3.3.1 Method of Optimal Directions Algorithm................................ 31

3.3.2. K-SVD Algorithm................................................. 32

Chapter 4. Image Fusion

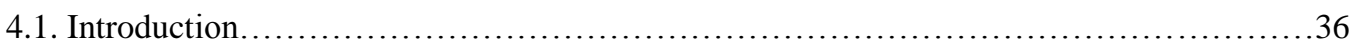

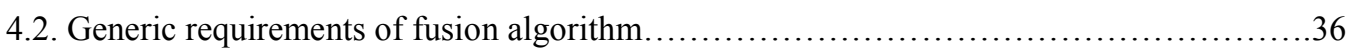

4.3. Preprocessing to satisfy Common Representation Format............................... 37.

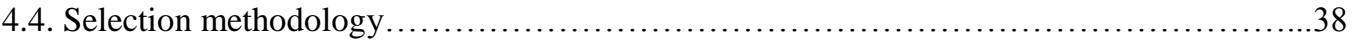

4.5. A brief review on area of application: Computed Tomography..........................39

4.5.1. Historical Milestones of Computed Tomography................................40

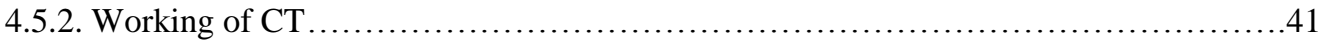

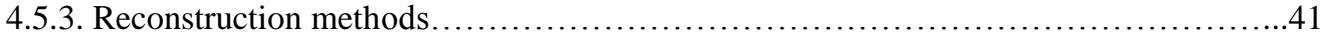

4.5.4. Computed Tomography Dose...........................................42

4.5.5. Computed Tomography Noise...........................................45

4.6. Fusion Methodologies....................................................46

4.6.1. Image fusion using multi scale approach..................................46

4.6.2. 2D-Discrete Wavelet Transform Fusion.....................................48

4.6.3. Principal Component Analysis based fusion........................................

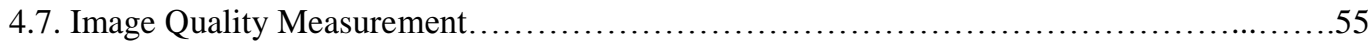

Chapter 5. Proposed Methodologies

5.1. Image Fusion Using Simultaneous Controlled St-OMP ............................59

5.2. Medical Image Fusion Based on Joint Sparse Method...............................64

5.3. Simultaneous medical image fusion and denoising using focused region in sparse domain..72

5.3.1 Dictionary learning and acquisition of initial fused image ........................... 72

5.3.2. Detection of focused region and fusion scheme................................. 75

Chapter 6. Results and Discussions

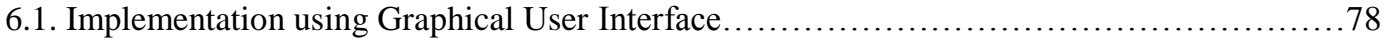

6.2. Experimental setup and results of Simultaneous Controlled St-OMP ......................80

6.3. Experimental setup and Results of Contribution II and Contribution III...................92

6.4. Integration of unregistered images using focused region fusion.........................98 
Chapter 7. Conclusion and Future Works

7.1. Image fusion with simultaneous controlled orthogonal matching pursuit.................101

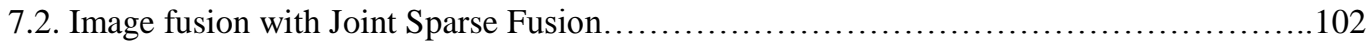

7.3. Fusion based on focused Vector...................................................... 102 


\section{List of Tables}

Table 2.1 Pseudo algorithm of Matching Pursuit

Table 2.2 Pseudo algorithm of Weak Matching Pursuit

Table 2.3 Pseudo algorithm of Orthogonal Matching Pursuit

Table 2.4. Pseudo algorithm of Stage wise Orthogonal Matching Pursuit

Table 3.1. Pseudo algorithm of MOD dictionary learning strategy

Table 3.2. Pseudo Algorithm of KSVD Algorithm

Table 5.1. Pseudo algorithm of proposed Simultaneous Controlled St-OMP fusion

Table 5.2. Pseudo algorithm of Joint Sparse PCA fusion method

Table 6.1. Performance evaluation of proposed method for standard images quantitatively

Table 6.2. Performance evaluation of proposed method for CT images quantitatively

Table 6.3. Performance evaluation of proposed method for CT images quantitatively

Table 6.4. Performance evaluation of proposed method for CT images quantitatively 


\section{List of Figures}

Figure 1.1 Generic pixel level fusion processing chain

Figure 2.1. Depiction of sparse representation concept and the size of each matrix

Figure 2.3 Block diagram of St-OMP framework

Figure 3.1. Depiction of Simple $4 \times 4$ DCT Dictionary of 16 atoms and $8 \times 8$ DCT Dictionary of 64 atoms

Figure 3.2. DCT dictionary with $8 \times 8$ patches

Figure 3.3. (a) DWT having one decomposition level (b) DWT having three decomposition levels and (c) Haar Dictionary basis

Figure 3.4. Left image representing the Wavelets associated with the orientation of DTCWT and Right image represents the Overcomplete DTCWT dictionary

Figure 4.1. Source images to be integrated: (a) Visual image (b) infrared image.

Figure 4.2. Principle of Computed Tomography

Figure 4.1. Source images to be integrated: (a) Visual image (b) infrared image.

Figure 4.2. Principle of Computed Tomography

Figure.4.3. 2D Decomposition; level=3

Figure.4.4. 2D DWT Fusion framework

Figure.4.5. 2D DTCWT Fusion framework

Figure.4.6. PCA Fusion framework

Figure 5.1. Framework of proposed Simultaneous Controlled St-OMP fusion

Figure 5.2. Framework of Joint Sparse PCA fusion methodology 
Figure 6.1. Screenshot of Graphical User Interface for pixel level fusion method

Figure 6.2. Displaying the fusion result and difference from reference of standard images using 1. Multifocus source images and a Reference image 2. OMP-DCT, 3.OMP-MDCT, 4. SOMPMDCT, 5. SCSt-OMP utilizing Overcomplete DCT and 6. SCSt-OMP utilizing MDCT.

Figure 6.3. Displaying the fusion result and contrast map using 1. Low dose image, 2.Highdose image as reference, 3. OMP-DCT, 4.OMP-MDCT, 5. SOMP-MDCT, 6. SCSt-OMP utilizing Overcomplete DCT, 7. SCSt-OMP utilizing MDCT, 8. Joint Sparse PCA model (Contribution II), 9. Focussed Region fusion.

Figure 6.4. Displaying the fusion result and contrast map using 1. Low dose image, 2.Highdose image as reference, 3. OMP-DCT, 4.OMP-MDCT, 5. SOMP-MDCT, 6. SCSt-OMP utilizing Overcomplete DCT and 7. SCSt-OMP utilizing MDCT.

Figure 6.5. Displaying the fusion result and contrast map using 1. Low dose image, 2.Medium dose image, 3. High dose image, 4.Focussed Region Fusion, 5. CSt-OMP fusion, 6. Joint Sparse PCA fusion

Figure 6.6. The overcomplete dictionaries: (a) Overcomplete DCT (b) MDCT Dictionary (c) Trained Overcomplete dictionary

Figure 6.7. The fusion result and Contrast map of fusing two Cone Beam CTs (CBCT). 


\section{List of Acronyms}

PSNR: Peak Signal to Noise Ratio

CNR: Contrast to Noise Ratio

DCT: Discrete Cosine Transform

DL: Dictionary Learning

KSVD: Generalized K -Means Singular Value Decomposition

LASSO: Least Absolute Shrinkage and Selection Operator

MDCT: Modified Discrete Cosine Transform

MOD: Method of Optimal Directions

MP: Matching Pursuit

OMP: Orthogonal Matching Pursuit

St-OMP: Stage wise Orthogonal Matching Pursuit

CSt-OMP: Controlled Stage wise Orthogonal Matching Pursuit

SOMP: Simultaneous Orthogonal Matching Pursuit

SCSt-OMP: Simultaneous Controlled Stage wise Orthogonal Matching Pursuit

PI-DCT: Phase Included Discrete Cosine Transform

PCA: Principal Component Analysis 


\section{Chapter 1. Introduction}

With the tremendous growth in imaging sensor technology in various applications such as remote sensing, automatic object detection, computer vision and medical imaging, the need for effective fusion algorithms is inevitable. This is due to the fact that image captured by different sensors carry complementary information. Even the images captured by the same sensor over an extended period of time need not contain same details. Image fusion is the process of integrating relevant information from source images to single composite image. And the composite image must carry all the salient details that can describe the scene better than any of the source images. This integrated image can be used for machine perception or human perception.

\subsection{Background}

Extraordinary advances in medical scanning technology have revolutionized clinical diagnosis. The arrival of various imaging modalities provides invaluable information about pathology and anatomy of patients. Optimal exploitation of all the critical information necessary for diagnosis in the clinical treatment is a challenging task. There are many technical hurdles to be handled while fusing medical images. The fast and detailed insight in to the patient's body noninvasively makes computer tomography tremendously widespread in today's healthcare 
system. Technological advances of CT in usage of radiological diagnosis and treatment makes it an indispensible tool in clinical examinations.

\subsection{Problem statement}

Drastic increase in the number of CT examinations led to the increase in radiation dose in the patients. Even though the clinical diagnosis is precise, increase in dose has created anxiety among the radiological community. Hence the demand for reducing the dose arises but this might impact the quality of the image. There is a tradeoff between noise and dose. Decreasing the dosage leads to increase in noise that might hinder the details needed for clinical diagnosis. Reducing the dose without compromising the image quality is a challenging task. The objective is to improve the quality of low dose images equivalent to that of image generated with a high dose. For instance, usage of low pass filter might remove high frequency noise but some small content might be lost and edges will be blurred. Hence there is a compromise between image quality and dose. Integrating multiple low dose and medium dose data of the same region of interest in to a coherent composite image, assuring all salient information without any loss of diagnostically relevant content would be an optimal way to enhance and preserve the important information and reduce the noise. Therefore a great care has to be given while developing fusion algorithm for this specific application for efficient expert interaction.

The following are the main goals in fusing low dose and medium dose CT data: 1 . Minimize noise and improve PSNR and CNR; 2. Improve composite image that provides an invaluable roadmap for precise clinical diagnosis than any of the low dose and medium dose images; Objective of this thesis is to provide a technical solution for integrating and denoising low dose and medium dose images simultaneously.

\subsection{Techniques adopted}

Sparse representation of any image requires only limited number of prototypes to represent the underlying salient features of the image. Any image can be represented as sparse linear combination of basis functions in a dictionary and each basis function is gmcalled an atom. Hence the fused image can be represented by sparse coefficients and well designed overcomplete

dictionaries. Signal can be approximated through many optimal iterative algorithms utilizing a 
well designed dictionary. The dictionaries can be analytical or adaptive. Analytical dictionaries are pre-specified dictionaries where the atoms are created using predefined basis functions like curvelets, cosine functions, bandlets, etc. These fixed dictionaries are simple to implement but they are not specialized in representing all images. But on the other hand, in adaptive dictionaries, the atoms are generated and updated based on the training data. Learning algorithms deal with this problem to find an optimal solution.

Multiple low dose and medium dose images of the same scene can be seen as an ensemble of intercorrelated images. Firstly, the data in the ensemble is sparsely represented using an overcomplete dictionary as common and innovative sparse components. It is a well known fact that common feature has more chances to be clean and innovative components might contain noise. Then an appropriate fusion rule is applied to combine common and innovative components in sparse domain to enhance the underlying sparse information and simultaneously the innovative components are denoised. Finally the fused image is recovered from the composite vector.

\subsection{Framework and Requirements}

Through image fusion synergy process, the source data can be fused to obtain a composite image with an improved quality, reduced uncertainty and increased reliability. Generalized fusion processing chain is shown in Figure 1.1. Multiple CT images are fused only when they "speak a common language". Common representational block makes sure that the preliminary condition for fusion of source images having common representation format is satisfied. Hence before fusion, first the source images have to be aligned spatially in to the same geometric base, temporally aligned to a common time and radiometrically calibrated in to a common measurement scale. Image fusion block integrates the aligned data together into a composite one. There are various fusion operations: 1. Pixel operations, 2. Sub-space methodology and 3. Multi-scale fusion methodology.

Requirements for effective fusion algorithm are: 1. Composite image should preserve all the necessary information, 2. The fused image should be artifact free, 3. Framework should be rotational and shift invariant and 4. Algorithm should be temporally stable and consistent. 


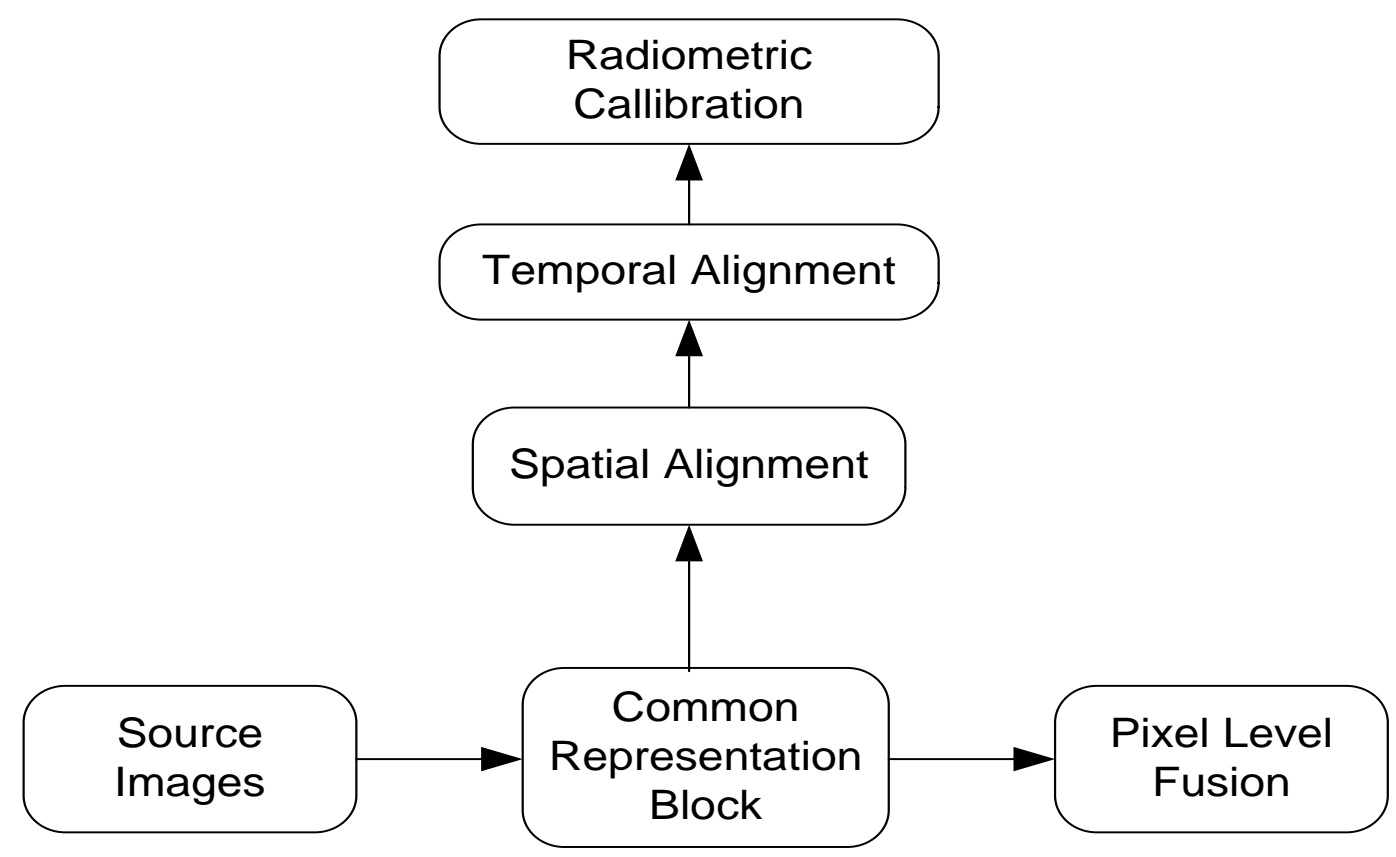

Figure 1.1 Generic pixel level fusion processing chain

\subsection{Major contributions}

Motivated by the need for processing the low dose images and to address the challenges and key technical issues faced while fusing low dose and medium dose images, our research demonstrates the use of pixel level fusion in sparse domain for integrating multiple dose images.

Contribution I: Simultaneous Controlled Stage-wise Orthogonal Matching Pursuit (SCStOMP) algorithm, which is a new efficient simultaneous sparse coding iterative framework, is developed to fasten the approximation process of joint sparse model.

Contribution II: A hybrid Joint Sparse PCA model is proposed in [1.] that can simultaneously fuse the common and innovative component using different fusion rules and denoise the source innovative components.

Contribution III: Development of a novel fusion algorithm [2.], applicable for both noisy and clean images, that determines the focused sparse vector from initial fused image and fuse the common and innovative components of focused sparse vector, initial fused image and the 
ensemble. The signals in the ensemble are represented sparsely by adopting SCSt-OMP (Contribution I) utilizing the dictionary trained from high dose data set.

\subsection{Overview of the thesis}

This thesis is organized as follow: Sparse representation techniques that are used to represent the source data effectively for clinical diagnosis is presented in chapter 2 . All the algorithms in chapter 2 are constructed based on assumption that the dictionary is already known. Well designed dictionaries and effective dictionary learning methodologies for better interpretation of images are described in chapter 3. Computed tomography technique with the assembly of image fusion methodologies and image quality measurement is rendered in chapter 4. In chapter 5, our contributions that were developed to fuse low dose and medium dose CT images are presented in detail. Chapter 6 explores the quantitative and qualitative analysis of composite images obtained from proposed methodologies. The efficiency of the proposed methods is well demonstrated by comparing with other state-of-the-art fusion methods. Finally, in chapter 7, the thesis is concluded with a summary and avenues for extending our proposed work. 


\section{Chapter 2. Sparse Representation}

\subsection{Introduction to Sparsity}

The ultimate goal of transform coding algorithms is to find a transform that will represent any signal with few coefficients. Previously Orthogonal Transform [1] is applied for generating sparse approximation of the signal but finding the exact representation is almost impossible. An example for transform domain representations is the Fourier Transform with vector space having Fourier orthogonal basis since they can represent periodic functions sparsely.

The word "Sparse" means quantitative property of a vector. Sparsity is the measure of number of non-zero coefficients in a vector. Computational efficiency of multiplication of non sparse vector and matrix is better compared to the multiplication of sparse vector and matrix.

Considering a dictionary $D \in \mathbb{C}^{n \times m}$ to be over complete $(n \ll m)$ for the given signal $b \in \mathbb{C}^{n}$, solution $x \in \mathbb{C}^{m}$ obtained from the underdetermined linear system shown in figure 2.1 and the equation is given by: 
$b=D x$

Sparse vectors are memory efficient way of mapping position and value of entries.

$\|x\|_{0}=\#\left\{j: x_{j} \neq 0, j=1,2, \ldots, n\right\}$

$\|x\|_{0}$ defines the number of non-zero entries of the vector. $\|x\|_{0}$ represents the $l_{0}$-norm of the vector $x$. As $l_{0}$-norm informally represents the number of non-zero entries, they are focused to find the sparsest solution for any under determined linear system. Many image processing applications try to obtain sparsest solution by minimizing the number of non-zero entries by applying certain constraints. This problem is called $l_{0}$-minimization and is formulated as:

$\min \|x\|_{0}$ subject to $b=D x$

Solution to this problem is unattainable due to the lack of mathematical representation of $l_{0^{-}}$ norm.

Mathematically, $l_{n}$-norm is formulated as:

$\|x\|_{n}=\sqrt[n]{\sum_{j}\left|x_{j}\right|}$

$\|x\|_{n}$ is called norm. Norm is a function that represents the length of each vector in vector space. Norm has many profiles like Euclidean distance, minimum square error, etc.

Representation of $l_{1}$-norm following the definition of $\|x\|_{n}$-norm is:

$\|x\|_{1}=\sum_{j}\left|x_{j}\right|$

Mean absolute error $\epsilon_{m}$ of two vectors $x_{1}$ and $x_{2}$ of size $n$ can be computed by:

$\epsilon_{m}=\frac{1}{n}\left\|x_{1}-x_{2}\right\|_{1}$

This is the most well known norm and is demanding in image processing applications. By the definition of norm, $l_{2}$-norm or Euclidean norm can be represented as:

$\|x\|_{2}=\sqrt{\sum_{j}\left|x_{j}^{2}\right|}$ 
$l_{2}$-norm calculated for difference between two vectors is given as:

$E_{d}=\sqrt{\frac{1}{n}\left\|x_{1}-x_{2}\right\|_{2}^{2} \sum_{j}\left|x_{j}{ }^{2}\right|}$

$E_{d}$ is called the Euclidean distance between vectors $x_{1}$ and $x_{2}$ of size $n \times 1$. Mean Square Error (MSE) is one of the important quantitative measurements to compute Peak Signal to Noise Ratio (PSNR) of the denoised signal.

\subsection{Regularization}

This problem of $l_{2}$-minimization is formulated as:

$\min \|x\|_{2}$ subject to $b=D x$

Considering $\mathrm{D}$ to be a full rank matrix, this underdetermined linear system is likely to have infinite number of solutions. Computation is highly complex to draw the best possible solution for this problem. Lagrange multipliers $\lambda$ are used to make this cumbersome process workable.

$\mathcal{L}(x)=\|x\|_{2}^{2}+\lambda^{T}(D x-b)$

Optimal solution is obtained by equating the derivative of Lagrangian $\mathcal{L}(x)$ to zero:

$\hat{x}_{o p t}=-\frac{1}{2} D^{T} \lambda$

Substituting this solution in constraint equation gives:

$-\frac{1}{2} D D^{T} \lambda=b$

$\lambda=-2\left(D D^{T}\right)^{-1} b$

Optimal solution of $l_{2}$-optimization is obtained by substituting Lagrange multiplier $\lambda$ in equation (2.10)

$\hat{x}_{o p t}=D^{T}\left(D D^{T}\right)^{-1} b=D^{+} b$ 
This problem is called the least square optimization problem and the optimal solution is instantly obtained by solving this equation. This equation is popularly called Moore-Penrose Pseudoinverse [2]. Unique solution is very hard to find because of the smooth nature of Euclidean norm even though it is computationally simple.

This problem is $l_{1}$-minimization and is formulated as:

$\min \|x\|_{1}$ subject to $b=D x$

As $l_{1}$-norm is not smooth in nature, it has the tendency to draw best sparse solution. Thus unique best solution from the infinitely many solutions can be drawn. Since it is mathematically complex to draw from pool of infinite solutions, complex optimization algorithms are developed to easily select the best unique solution.
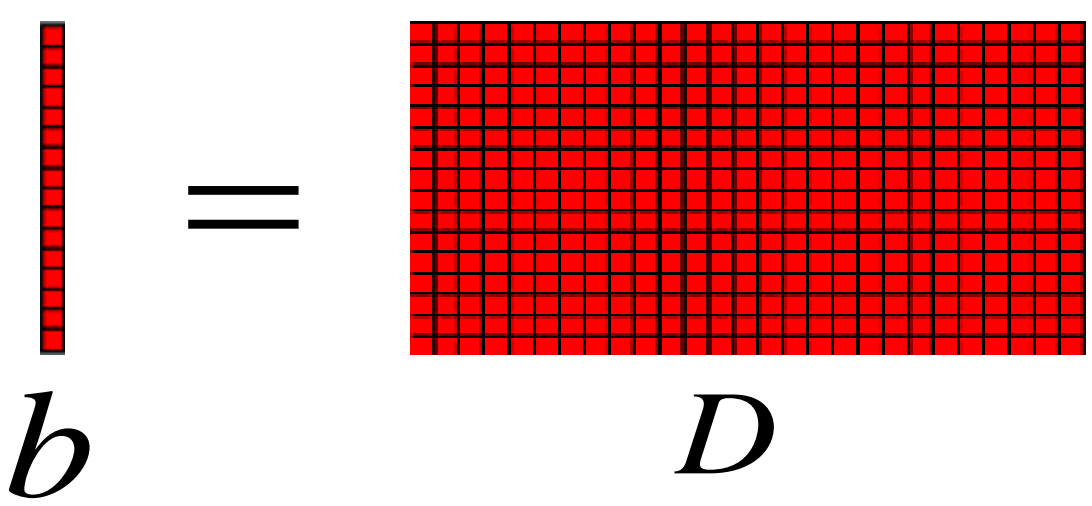

Figure 2.1. Depiction of sparse representation concept and the size of each matrix

\subsection{Spare Approximation}

1) All the sparse approximation algorithms are constructed by assuming the dictionary $D$ to be known. 
The problem defined in (2.3) will have infinitely many solutions if $b$ is the span of dictionary matrix $D$. The solution $x$ cannot reproduce the signal $\mathrm{b}$ exactly since the equality constraint is strict.

2) Considering $\mathrm{D}$ and $\mathrm{b}$ to be same as problem (2.15), applying a condition to find the solution is given as:

$\hat{x}=\operatorname{argmin}_{x}\|x\|_{1}$ subject to $\|D x-b\|_{2}<\epsilon \quad \epsilon \in \mathbb{C}$

Where $\epsilon$ is the global representation error and $\epsilon>0$. For this problem, the value of $x$ is flexible since the solution is no longer required to reproduce the signal exactly and the constraint is not too strict.

\subsection{Examples of sparse representation and approximation}

Sparse approximation of a randomly generated vector utilizing randomly generated overcomplete matrix is given as:
1) $\left(\begin{array}{lllll}0.8147 & 0.6324 & 0.9575 & 0.9572 & 0.4218 \\ 0.9058 & 0.0975 & 0.9649 & 0.4854 & 0.9157 \\ 0.1270 & 0.2785 & 0.1576 & 0.8003 & 0.7922 \\ 0.9134 & 0.5469 & 0.9706 & 0.1419 & 0.9595\end{array}\right)\left(\begin{array}{l}0 \\ 1.1464 \\ 0 \\ 0 \\ 0.2400\end{array}\right)=\left(\begin{array}{l}0.6557 \\ 0.0357 \\ 0.8491 \\ 0.9340\end{array}\right)$
2) $\left(\begin{array}{lllll}0.8147 & 0.6324 & 0.9575 & 0.9572 & 0.4218 \\ 0.9058 & 0.0975 & 0.9649 & 0.4854 & 0.9157 \\ 0.1270 & 0.2785 & 0.1576 & 0.8003 & 0.7922 \\ 0.9134 & 0.5469 & 0.9706 & 0.1419 & 0.9595\end{array}\right)\left(\begin{array}{l}0 \\ 0 \\ 0 \\ 0 \\ 0.7323\end{array}\right)=\left(\begin{array}{l}0.6557 \\ 0.0357 \\ 0.8491 \\ 0.9340\end{array}\right)$

The error in second example is 0.8058 which is lower than the length of the source vector which is 1.4229 . First example has two sparse coefficients and the error is 0.4878 which is much lower than the example with only one sparse coefficient. 


\subsection{Uncertainty}

Consider the dictionary $D$ to be the concatenation of two orthogonal bases $\Phi$ and $\Psi$. Simply the dictionary $D$ is considered as the composition of identity matrix and Fourier matrix. For the defined underdetermined system, there are many solutions that represent the signal as a superposition of columns from the concatenated matrices that are spikes and sinusoids:

$[\Phi, \Psi] c=b$

Optimal sparse solution represents the signal with fewer non-zero entries that is the superposition of fewer sinusoids and spikes.

Uncertainty principle states that any function and Fourier transform of the same function cannot be sharply localized [61].

$$
\int_{-\infty}^{\infty} x^{2}|f(x)|^{2} d x \cdot \int_{-\infty}^{\infty} \omega^{2}|F(\omega)|^{2} d \omega \geq \frac{1}{2}
$$

Measuring the concentration in terms of standard deviation defined on $\mathbb{C}$ and hence:

$\int_{-\infty}^{\infty}|f(x)|^{2} d x=1$

Signal can be represented as the linear combination of columns of identity matrix or Fourier matrix as:

$b=\Psi \alpha_{t}=\Phi \alpha_{\mathrm{f}}$

Where $\alpha_{t}$ is the time-domain representation of signal $\mathrm{b}$ and $\alpha_{f}$ is the frequency domain representation of signal b. For the pair of considered orthogonal bases either $\alpha_{t}$ or $\alpha_{f}$ can be sparse. Mutual coherence of the dictionary $D$ is proximity between the two orthogonal bases which is:

$\mu(D)=\max _{i \geq 1, j \leq n}\left|\Psi_{i}^{T} \Phi_{j}\right|$ 
If $\Psi$ is a unit matrix and $\Phi$ is a Fourier matrix, then mutual coherence [3] is $\frac{1}{\sqrt{n}}$. Both $\alpha_{t}$ and $\alpha_{f}$ cannot be sparse at the same time as $\mu(D)$ is small. $\mu(D)$ can also be determined as the non diagonal entry of gram matrix $G=D^{T} D$ as:

$\mu(D)=\max _{i \geq 1, j \leq K, i \neq j}\left|G_{i, j}\right|$

\subsection{Uniqueness of sparse representation}

In order to narrow the choice of well defined solution, certain constraints are applied to this problem.

Sparsest possible solution is drawn if

$$
\|x\|_{0}<\frac{\operatorname{spark}(D)}{2}
$$

$\operatorname{Spark}(D)$ [4] is the smallest number of linearly dependent vectors of D. Consider $\exists x_{1}, x_{2}$, the sparse representation of the signal $b$ can be represented as:

$D x_{1}=D x_{2}=b\left(x_{1} \neq x_{2}\right)$

$D x_{1}-D x_{2}=0$

$\left\|x_{1}-x_{2}\right\|<\operatorname{Spark}(D)$

But the minimality nature of $\operatorname{Spark}(D)$ is contradicted. Hence the unique representation cannot be guaranteed. But it is only upto a bounded deviation hence one can get closer to such matrices with this scheme.

\subsection{Necessity of sparse approximation}

Sparse approximation algorithms are widely used in image and signal processing applications. Obtaining the sparse approximation is not as simple as an abstract mathematical problem. It is very challenging to store vectors containing large amount of data and computation also becomes very tedious since Graphics Processing Units (GPUs) carry very limited amount of quickly accessible memory. Sparse approximation techniques effectively store sparse vectors. Data can be analyzed by the reciprocality of sparse vectors. A sparse matrix allows processes to take 
advantage of background or zero elements and represents the underlying salient elements. Nature of Sparsity of any matrix depends upon its structure and application.

\subsection{Sparse coding algorithms}

The objective of sparse problem $P_{0}$ is to obtain fewer coefficients for the given constraint function (2.2). Obtaining exact solution for this problem is NP-hard. There are various iterative algorithms which are used to find the objective function. During each iteration, appropriate column vectors are chosen for obtaining the optimum global solution.

\subsubsection{LASSO and Basis Pursuit}

Basis Pursuit (BP) is used to substitute a complex sparse problem by an easier optimization problem. Equation (2.2) is the formal way of defining the sparse problem. The main difficulty with this sparse problem is the $l_{0}$-norm. BP replaces $l_{0}$-norm with $l_{1}$ constraint for solving the problem with relative ease and can be defined as:

$\min \|x\|_{1}$ subject to $b=D x$

Least Absolute Shrinkage and Selection Operator (LASSO) also known as BP de-noising introduced in [5] replaces the sparse problem by a convex problem for efficient shrinkage and variable selection in linear models. Derivative of the objective function is not possible due to the $l_{1}$ constraint. This motivates the need for special optimization techniques. Grafting, which is the stage-wise gradient descent algorithm, is developed in [6] to solve LASSO problem. However this method is not computationally efficient. After many attempts to solve the issues faced while solving the problem, gradient LASSO algorithm was proposed by [7] overcomes these issues. This converges to global optimum and can efficiently handle large dimensional data set since this algorithm does not require matrix inversion. LASSO problem can be mathematically formulated as:

$\min \|D x-b\|_{2}$ subject to $\|x\|_{1}<\lambda$

\subsubsection{Greedy Algorithms}


Unique solution is obtained when the dictionary $D$ has $\operatorname{spark}(D)>2$, and the value of the optimization problem $P_{0}$ is 1 . Identification of columns are done when the signal is a scalar multiple of those identified columns. Objective is to minimize the error $\epsilon(i)$ and is represented mathematically as:

$\epsilon(i)=\min _{z_{i}}\left\|d_{i} z_{i}-b\right\|_{2}^{2}$

Minimizing the representation error leads to:

$z_{j}^{*}=\frac{d_{i}^{T} b}{\left\|d_{i}\right\|_{2}^{2}}$

Substituting $z_{j}^{*}$ in error function leads to:

$\epsilon(i)=\left\|\frac{d_{i}^{T} b}{\left\|d_{i}\right\|_{2}^{2}} d_{i}-b\right\|_{2}^{2}$

The best solution is obtained when error is zero. Greedy pursuit iterative algorithm [8] constructs approximation iteratively reducing the residual error. Locally optimum solution is identified at each iteration step. Properly identifying the local optimal solutions at each step will result in a global optimal solution.

\subsubsection{Matching Pursuit with Time-Frequency Dictionaries}

This algorithm is introduced by [9] which provides extremely flexible signal representation and can decompose any signal in to a linear expansion of waveforms that are well localized both in time and frequency. The property of decomposition varies with respect to the choice of timefrequency atoms. This iterative algorithm decomposes any signal in to waveforms that well describe the time-frequency property of the signal. Best represented signal structures that correlate well with the given dictionary are detected and isolated by this algorithm.

Table 2.1 Pseudo algorithm of Matching Pursuit [9]

Objective: Construct an approximation for the problem $\mathrm{P}_{0}$

$\left(\mathrm{P}_{0}\right): \min \|x\|_{0}$ subject to $b=D x$ 
User defined input parameters: Dictionary $D$, Signal $b$ and error threshold $\epsilon_{0}$.

Initial conditions: $\mathrm{k}=0$, start by setting

Intial residual: $r^{0}=b-D x^{0}\left(\right.$ set initial solution $\left.x^{0}=0\right)$

Initial index set: $S^{0}=\varnothing$

Iteration process: $\mathrm{k}=\mathrm{k}++$ (incrementing)

Sweep stage: Error computation $\epsilon(i)=\min _{z_{i}}\left\|d_{i} z_{i}-r^{k-1}\right\|_{2}^{2}$ for all $i$. Minimizing error leads to the optimal choice $z_{i}^{*}=\frac{d_{i}^{T} r^{k-1}}{\left\|d_{i}\right\|_{2}^{2}}$.

Update solution stage: If the inner product between row vectors $d_{k}$ of dictionary $\mathrm{D}$ and residual is $d_{i}$ and is maximum, then assign $s_{t}=i$. Update the set $S_{t}=S_{t-1} \cup\left\{s_{t}\right\}$. Set $x^{k}=x^{k-1}$ and update the entry $x^{k}(i)=x^{k}(i)+z_{i}^{*}$

Update residual stage: compute the residual $r^{k}=b-D x^{k}=r^{k-1}-z_{i_{0}}^{*} d_{i_{0}}$.

Stopping condition: check the stopping criterion. If $\left\|r^{k}\right\|_{2}<\epsilon_{0}$, global optimum solution is obtained; Otherwise, proceed the iteration process again.

Output: $x^{k}$ is the global optimum solution obtained after $k$ iterations.

\subsubsection{Weak Matching Pursuit algorithm}

Simplified version of MP algorithm is the weak matching pursuit algorithm [9]. Greedy selection step in weak matching pursuit algorithm is relaxed by allowing a suboptimal choice of the next element to be added to the support set. Instead of looking for the largest inner-product value, we select the first found that exceeds $t$-weaker threshold. Using the Cauchy-Schuartz inequality,

$\frac{\left(d_{i}^{T} r^{k-1}\right)^{2}}{\left\|d_{i}\right\|_{2}^{2}} \leq \max _{1 \leq i \leq m} \frac{\left(d_{i}^{T} r^{k-1}\right)^{2}}{\left\|d_{i}\right\|_{2}^{2}} \leq\left\|r^{k-1}\right\|_{2}^{2}$ 
At the beginning of the sweep stage, $\left\|r^{k-1}\right\|_{2}^{2}$ is computed and we select $s_{t}$ that produces the smallest error $\epsilon(i) . s_{t}$ is chose by:

$\frac{\left(d_{i_{0}}^{T} r^{k-1}\right)^{2}}{\left\|d_{i_{0}}\right\|_{2}^{2}} \geq t^{2} \cdot\left\|r^{k-1}\right\|_{2}^{2} \geq t^{2} \cdot \max _{1 \leq i \leq m} \frac{\left(d_{i}^{T} r^{k-1}\right)^{2}}{\left\|d_{i}\right\|_{2}^{2}}$

Table 2.2 Pseudo algorithm of Weak Matching Pursuit [9]

Objective: Construct an approximation for the problem $\mathrm{P}_{0}$

$\left(\mathrm{P}_{0}\right): \min \|x\|_{0}$ subject to $b=D x$.

User defined input parameters: Dictionary $\mathrm{D}$, Signal $\mathrm{b}$ and error threshold $\epsilon_{0}$.

Initial conditions: $\mathrm{k}=0$, start by setting

Initial residual: $r^{0}=b-D x^{0}\left(\right.$ set initial solution $\left.x^{0}=0\right)$

Initial index set: $S^{0}=\varnothing$

Iteration process: $\mathrm{k}=\mathrm{k}++$ (incrementing)

Sweep stage: Error computation $\epsilon(i)=\min _{z_{i}}\left\|d_{i} z_{i}-r^{k-1}\right\|_{2}^{2}$ for all $i$. Minimizing error leads to the optimal choice $z_{i}^{*}=\frac{d_{i}^{T} r^{k-1}}{\left\|d_{i}\right\|_{2}^{2}}$. When $\frac{\left|d_{i}^{T} r^{k-1}\right|}{\left\|d_{i}\right\|_{2}} \geq t \cdot\left\|r^{k-1}\right\|_{2}$, stop the sweep.

Update solution stage: use $s_{t}$ from sweep stage and assign $s_{t}=i$. Update the set $S_{t}=S_{t-1} \mathrm{U}$ $\left\{s_{t}\right\}$. Set $x^{k}=x^{k-1}$ and update the entry $x^{k}(i)=x^{k}(i)+z_{i}^{*}$

Update residual stage: compute the residual $r^{k}=b-D x^{k}=r^{k-1}-z_{i_{0}}^{*} d_{i_{0}}$.

Stopping condition: check the stopping criterion. If $\left\|r^{k}\right\|_{2}<\epsilon_{0}$, global optimum solution is obtained; Otherwise, proceed the iteration process again.

Output: $x^{k}$ is the global optimum solution obtained after $k$ iterations.

\subsubsection{Orthogonal Matching Pursuit algorithm}


The strategy of Orthogonal Matching pursuit [8] [10] [11] makes the approximation computationally efficient by abandoning exhaustive searches. Orthogonalization makes this algorithm different from simple matching pursuit algorithm. Update provisional stage, which updates the residual vector at each iteration using least square step, enhances the approximation. The columns in dictionary D and residual should be orthogonal and already selected atoms will not be selected in the following iterations.

Table 2.3 Pseudo algorithm of Orthogonal Matching Pursuit [8]

Objective: Construct an approximation for the problem $\mathrm{P}_{0}$

$\left(\mathrm{P}_{0}\right): \min \|x\|_{0}$ subject to $b=D x$.

User defined input parameters: Dictionary $D$, Signal $b$ and error threshold $\epsilon_{0}$.

Initial conditions: $\mathrm{k}=0$, start by setting

Initial residual: $r^{0}=b-D x^{0}\left(\right.$ set initial solution $\left.x^{0}=0\right)$

Initial index set: $S^{0}=\varnothing$

Iteration process: $\mathrm{k}=\mathrm{k}++$ (incrementing)

Sweep stage: Error computation $\epsilon(i)=\min _{z_{i}}\left\|d_{i} z_{i}-r^{k-1}\right\|_{2}^{2}$ for all $i$. Minimizing error leads to the optimal choice $z_{j}^{*}=\frac{d_{i}^{T} r^{k-1}}{\left\|d_{i}\right\|_{2}^{2}}$.

Update solution stage: If the inner product between row vectors $d_{k}$ of dictionary $\mathrm{D}$ and residual is $d_{i}$ and is maximum, then assign $s_{t}=i$. Update the set $S_{t}=S_{t-1} \cup\left\{s_{t}\right\}$. Find the minimizer $x^{k}$ the term $\|D x-b\|_{2}^{2}$ such that the support is $S^{k}$.

Update residual stage: compute the residual $r^{k}=b-D x^{k}$.

Stopping condition: check the stopping criterion. If $r^{k}<\epsilon_{0}$, global optimum solution is obtained; Otherwise, proceed the iteration process again.

Output: $x^{k}$ is the global optimum solution obtained after $k$ iterations. 
Error values in the sweep stage is represented as:

$\epsilon(i)=\min _{z_{i}}\left\|d_{i} z_{i}-r^{k-1}\right\|_{2}^{2}$

$\epsilon(i)=\left\|\frac{d_{i}^{T} r^{k-1}}{\left\|d_{i}\right\|_{2}^{2}} d_{i}-r^{k-1}\right\|_{2}^{2}$

Error can be minimized if the inner product between the residual $r^{k-1}$ and normalized dictionary matrix D is large.

\subsubsection{Stage wise Orthogonal Matching Pursuit}

To represent the signal sparsely faster and to solve the sparse representation optimization problem, iterative stage wise orthogonal matching pursuit method is proposed in [12]. This algorithm is extended from orthogonal matching pursuit algorithm. Global optimum solution is build by adding one vector at a time in OMP algorithm, whereas many vectors are extracted at each stage in St-OMP algorithm. Thus the number of iterations to obtain the global optimal solution is reduced. Firstly, the residual starts out being equal to the signal. In St-OMP method, the dot products of the signal to be approximated with the columns of dictionary are compared and the vectors above the set threshold value are selected for the next stage. Sparse approximation is done by applying least square method. The same process is repeated comparing every time with the residue vector. Set threshold value should select subset of atoms with higher correlation. Challenge lies in selecting optimum threshold value since different threshold produces different results. Hence the results of robust St-OMP will be more sparser for optimum threshold value. 


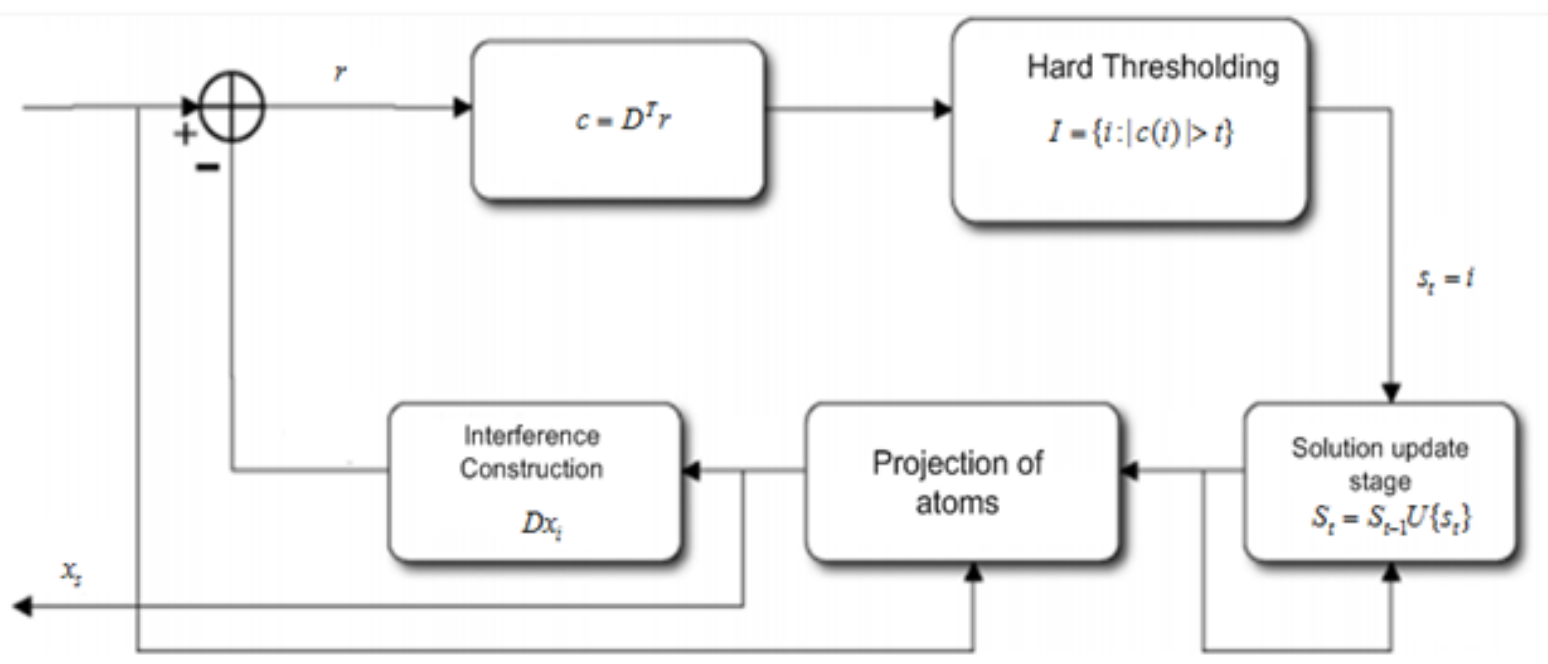

Figure 2.2 Block diagram of St-OMP framework

Table 2.4. Pseudo algorithm of Stage-wise Orthogonal Matching Pursuit [12]

Objective: Construct an approximation for the problem $\mathrm{P}_{0}$

$\left(\mathrm{P}_{0}\right): \min \|x\|_{0}$ subject to $b=D x$.

User defined input parameters: Dictionary $D$, Signal $b$ and error threshold $\epsilon_{0}$.

Initial conditions: $\mathrm{k}=0$, start by setting

Initial residual: $r^{0}=b-D x^{0}\left(\right.$ set initial solution $\left.x^{0}=0\right)$

Initial index set: $S^{0}=\varnothing$

Iteration process: $\mathrm{k}=\mathrm{k}++$ (incrementing)

Sweep stage: Error computation $\epsilon(i)=\min _{z_{i}}\left\|d_{i} z_{i}-r^{k-1}\right\|_{2}^{2}$ for all $i$. Minimizing error leads to the optimal choice $z_{j}^{*}=\frac{d_{i}^{T} r^{k-1}}{\left\|d_{i}\right\|_{2}^{2}}$. 
Update solution stage: If the inner product between row vectors $d_{k}$ of dictionary D and residual is $d_{i}$ and is maximum, then assign $s_{t}=i$. Update the set $S_{t}=S_{t-1} \cup\left\{s_{t}\right\}$. Find the minimizer $x^{k}$ and the term $\|D x-b\|_{2}^{2}$ such that the support is $S^{k}$.

Update residual stage: compute the residual $r^{k}=b-D x^{k}$.

Stopping condition: check the stopping criterion. If $r^{k}<\epsilon_{0}$, global optimum solution is obtained; Otherwise, proceed the iteration process again.

Output: $x^{k}$ is the global optimum solution obtained after $k$ iterations. 


\section{Chapter 3. Analytical and Adaptive Dictionaries}

Sparseland model is the basis for all the sparse image fusion algorithms described in this thesis. All the sparse approximation algorithms are developed under the fundamental consideration that the dictionary $D \in \mathbb{C}^{n \times m}$ is known. All the sparse coding algorithms rely heavily on costly computation with dictionary and signal on each iteration stage. Wise selection of dictionary results in redundant representation of signal efficiently. This chapter explains the nature of analytical and adaptive dictionaries.

\subsection{Comparison of analytical and adaptive dictionary}

Due to the rising need for efficient sparse representation, a variety of dictionaries have been constructed. There are mainly two sources for dictionary development: 1) Predefined mathematical model and 2) Realizations of training data. Analytical dictionaries such as wavelets, Discrete Cosine Transform [13], Contourlets [14], and many more are the wise dictionaries for fast implementation and better representation of signals in certain image processing applications. These pre-constructed dictionaries have the ability to sparsify only the signals having smooth boundaries. Even though these dictionaries, which are also called 
transforms, have simple implementation, they cannot be used to sparsify many type of signals of interest due to the analytical formulation.

By endorsing a learning point of view, learned based dictionaries overcome these limitations. On the other hand, trained dictionaries have generating atoms as a result of learning of empirical data instead from a theoretical model. They have the ability to sparsify any signal since they deliver high flexibility. The implementation is complex and it's computationally costly which do not expose the frequency information of the signal. Hence they are limited to low dimensional signals. In order to overcome the problems of learnt based dictionaries, these dictionaries are handled on small patches.

\subsection{Time-Frequency Dictionaries}

Transformation of a signal from spatial domain to frequency domain is necessary to reduce the observations representing the input data. Fourier transform is a widely used transform in various image processing tasks which decomposes an image into its sine and cosine components. This transformation exposes the frequency information of given data and divides the images based on the frequency information thereby performing the dimensionality reduction process naturally. It is known that Sinusoidal functions having different frequencies are orthogonal. Hence all the signals can be effectively represented as the linear combination of a set of orthogonal sinusoidal signals. During the earlier days, signal is represented as the combination of orthogonal basis using Fourier Transform due to the pair-wise orthogonality nature of sinusoidal functions. This has been widely given attention for extracting the frequency domain information of the signal.

Inner product of signal to be represented and its Fourier basis leads to the coefficients for effective representation of the signal which is nothing but the inverse Fourier transform.

$x(t)=\int_{-\infty}^{\infty} X(\xi) e^{j 2 \pi \xi t} d \xi$

Signal can be represented as the combination of orthogonal waveforms by Fourier basis. To approximate the signal, the Fourier basis creates $\mathrm{K}$ low frequency atoms of the dictionary. The signals described above are smooth and have less noise. 


\subsubsection{Discrete Cosine Transform}

DCT introduced in [13] is closely related to DFT but not complex. DCT decomposes the image based on differing frequencies. Most of the energy will be accumulated in the lower frequencies of the image. Decomposing the image based on frequency components, amount of details needed to describe the image well can be reduced by eliminating the high frequency coefficients. Fourier transformation works on the assumption that the signal is periodically extended which results in discontinuous boundaries. In order to overcome this and to produce real coefficients, the signal is assumed to be extended anti-symmetrically thereby making the boundary continuous. Due to the efficiency of DCT, it is much preferred in practical applications.

For a signal sequence, $X(n)$, its $l$ number of DCT coefficients $G_{x}(l)$ can be calculated by:

$$
G_{x}(l)=\frac{2}{N} \sum_{n=0}^{N-1} X(n) \cos \frac{(2 n+1) l \pi}{2 N} \text { where } l=1,2 \ldots \ldots, N-1
$$

And $0^{\text {th }}$ DCT coefficient is given by

$G_{x}(0)=\frac{\sqrt{2}}{N} \sum_{n=0}^{N-1} X(n)$

Inverse Cosine Transform can be represented in (3.4) as

$X(n)=\frac{1}{\sqrt{2}} G_{x}(0)+\sum_{n=1}^{N-1} G_{x}(l) \cos \frac{(2 n+1) l \pi}{2 N}$

Two dimensional extension follows straight forwardly from single dimensional DCT, the IDCT equation can be written as:

$X(n, u)=\sum_{l=0}^{N-1} \sum_{k=0}^{M-1} G_{x y}(l, k) \cos \frac{(2 n+1) l \pi}{2 N} \cos \frac{(2 u+1) k \pi}{2 M}$

\subsubsection{Overcomplete Discrete Cosine Transform}

We know that every signal can be sparsely represented when the dictionary forms a basis. When utilizing orthogonal dictionaries, salient coefficients are computed by calculating the inner product of the signal and atoms of orthogonal dictionary. Representation coefficients are 
computed by taking the inner product of signal and inverse of non-orthogonal dictionary and are referred to as bi-orthogonal dictionary.

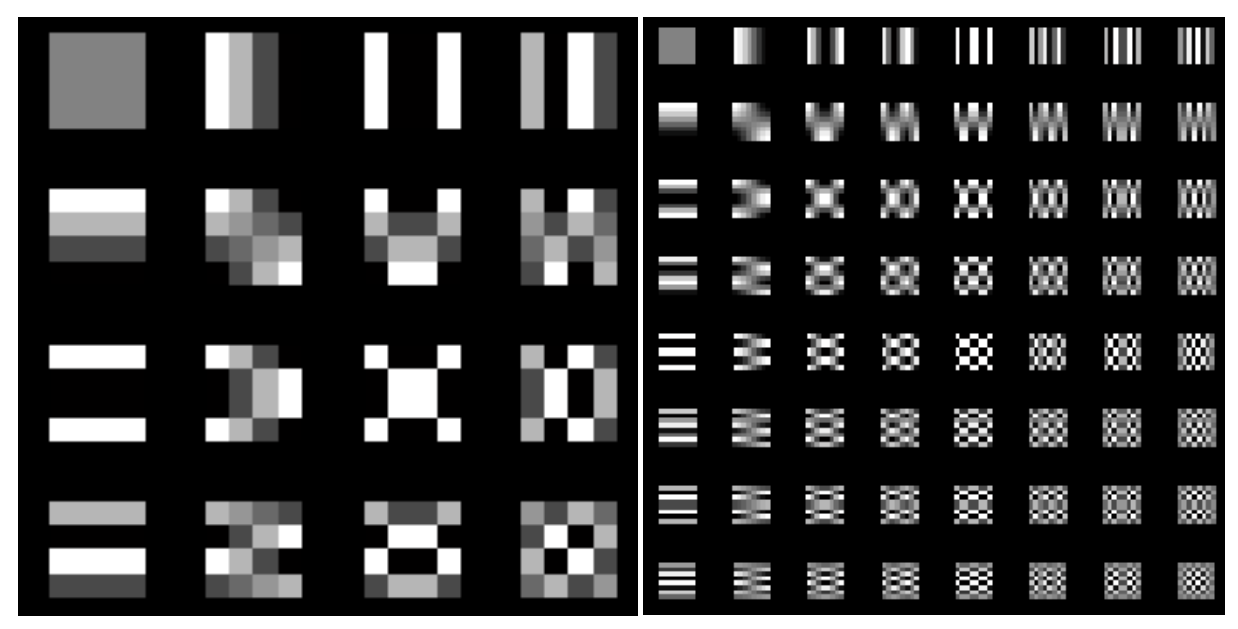

Figure 3.1. Depiction of Simple $4 \times 4$ DCT Dictionary of 16 atoms and $8 \times 8$ DCT Dictionary of 64 atoms

These dictionaries have limited ability to represent all the images. Due to the mathematical computational efficiency, these orthogonal and bi-orthogonal dictionaries were predominantly used until the development of a dictionary having more number of atoms than the signal dimension and is called overcomplete dictionary [16].

Considering the signal length of 64 , the overcomplete dictionary to be designed is presumed to have 256 columns. In non-overcomplete case, inner product of sample and atoms shows the representation coefficients. Transforming the sample into cosine space is done as shown in Figure 3.2. 


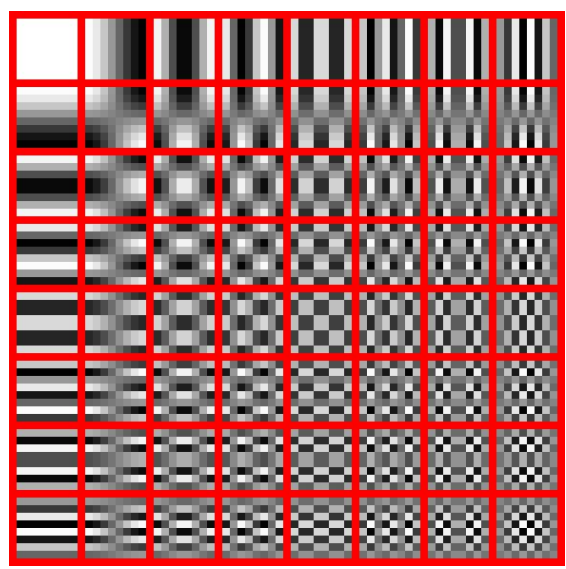

Figure 3.2. DCT dictionary with $8 \times 8$ patches

Two dimensional matrix $\tau$ which is transformed can be represented by

$$
\tau=\left[\begin{array}{cccc}
1 & 1 & \cdots & 1 \\
\cos \frac{\pi}{16} & \cos \frac{3 \pi}{16} & \ldots & \cos \frac{15 \pi}{16} \\
\vdots & \vdots & \ddots & \vdots \\
\cos \frac{7 \pi}{16} & \cos \frac{21 \pi}{16} & \cdots & \cos \frac{105 \pi}{16}
\end{array}\right]
$$

In between the orthogonal bases of $\tau$, non-orthogonal rows and columns are added thereby generating an overcomplete dictionary D of size $64 \times 256$.

$$
D=\left[\begin{array}{cccc}
1 & 1 & \cdots & 1 \\
\cos \frac{\pi}{32} & \cos \frac{3 \pi}{32} & \cdots & \cos \frac{15 \pi}{32} \\
\vdots & \vdots & \ddots & \vdots \\
\cos \frac{15 \pi}{32} & \cos \frac{45 \pi}{32} & \cdots & \cos \frac{225 \pi}{32}
\end{array}\right]
$$




\subsubsection{Overcomplete Haar Dictionary}

The idea of using Overcomplete Haar Dictionary is first proposed in [16].

One dimensional Haar transformed matrix of size $8 \times 8$ is shown below:

$$
\left[\begin{array}{cccccccr}
1 & 1 & 1 & 1 & 1 & 1 & 1 & 1 \\
1 & 1 & 1 & 1 & -1 & -1 & -1 & -1 \\
1 & 1 & -1 & -1 & 0 & 0 & 0 & 0 \\
0 & 0 & 0 & 0 & 1 & 1 & -1 & -1 \\
1 & -1 & 0 & 0 & 0 & 0 & 0 & 0 \\
0 & 0 & 1 & -1 & 0 & 0 & 0 & 0 \\
0 & 0 & 0 & 0 & 1 & -1 & 0 & 0 \\
0 & 0 & 0 & 0 & 0 & 0 & 1 & -1
\end{array}\right]
$$

Overcomplete Haar transformed matrix of size $64 \times 441$ is constructed by adding non orthogonal rows in between the orthogonal bases indicated by arrows. As usual the patches are vectorized and located at designated columns in a matrix. These columns are then normalized.

$\stackrel{\longrightarrow .}{. \longrightarrow}\left[\begin{array}{cccccccc}. \longrightarrow & 1 & 1 & 1 & 1 & 1 & 1 & 1 \\ 1 & 1 & 1 & 1 & -1 & -1 & -1 & -1 \\ -1 & 1 & 1 & 1 & 1 & -1 & -1 & -1 \\ -1 & -1 & 1 & 1 & 1 & 1 & -1 & -1 \\ -1 & -1 & -1 & 1 & 1 & 1 & 1 & -1 \\ 1 & 1 & -1 & -1 & 0 & 0 & 0 & 0 \\ 0 & 1 & 1 & -1 & -1 & 0 & 0 & 0 \\ 0 & 0 & 1 & 1 & -1 & -1 & 0 & 0 \\ 0 & 0 & 0 & 1 & 1 & -1 & -1 & 0 \\ 0 & 0 & 0 & 0 & 1 & 1 & -1 & -1 \\ -1 & 0 & 0 & 0 & 0 & 1 & 1 & -1 \\ -1 & -1 & 0 & 0 & 0 & 0 & 1 & 1 \\ 1 & -1 & -1 & 0 & 0 & 0 & 0 & 1 \\ 1 & -1 & 0 & 0 & 0 & 0 & 0 & 0 \\ 0 & 1 & -1 & 0 & 0 & 0 & 0 & 0 \\ 0 & 0 & 1 & -1 & 0 & 0 & 0 & 0 \\ 0 & 0 & 0 & 1 & -1 & 0 & 0 & 0 \\ 0 & 0 & 0 & 0 & 1 & -1 & 0 & 0 \\ 0 & 0 & 0 & 0 & 0 & 1 & -1 & 0 \\ 0 & 0 & 0 & 0 & 0 & 0 & 1 & -1 \\ -1 & 0 & 0 & 0 & 0 & 0 & 0 & 1\end{array}\right]$




\subsubsection{Continuous Wavelet Transform (CWT)}

Wavelet transform provides flexible time frequency window to adapt to the frequencies of different input images. This is proposed in [17] to overcome the problems of Fourier Transform.

CWT of the signal $X(t)$ can be expressed by

$X_{w}(x, y)=\frac{1}{\sqrt{y}} \int_{-\infty}^{\infty} X(t) \Psi\left(\frac{t-x}{y}\right) d t$

Where $y$ defines the scale which is a positive value and $x$ represents shift which is a real number. By shifting and scaling the basic wavelet $\Psi(t)$, wavelets are generated. This basic wavelet is well designed to be computationally efficient and easily reversible. CWT $X_{w}(x, y)$ of the given signal represents a high frequency component of the signal if the scale $y$ is larger. Undoubtedly, window size of $\Psi\left(\frac{t-x}{y}\right)$ will be smaller for large scale value.

\subsubsection{Continuous Wavelet Transform with Discrete Wavelet Coefficients (DC-CWT)}

DC-CWT overcomes the problem of intense implementation of CWT. Sampling the time scale period parameters $x$ and $y$ values are restricted as:

$x=n 2^{-m}$ and $y=2^{-m}$

CWT in this case is called DC-CWT and can be written as:

$X_{w}(x, y)=\frac{1}{\sqrt{2^{-m}}} \int_{-\infty}^{\infty} X(t) \Psi\left(\frac{t-n 2^{-m}}{2^{-m}}\right) d t$

Discrete scaling and discrete wavelet functions are given by:

$$
\begin{gathered}
\Psi(t)=2 \sum_{k} h_{k} \Phi(2 t-k) \\
\Phi(t)=2 \sum_{k} g_{k} \Phi(2 t-k)
\end{gathered}
$$

Where $h_{k}$ and $g_{k}$ are the FIR filters.

Convoluting $X_{w}(x, y)$ and $X_{w}(x, y-1)$ results in:

$\chi_{w}(x, y)=\sqrt{2} \sum_{k} g_{k} \chi_{w}(2 x+k, y+1) d t$ 
$X_{w}(x, y)=\sqrt{2} \sum_{k} h_{k} X_{w}(2 x+k, y+1) d t$

Scaling function $\Phi(t)$ can be understood as a low pass filter. Location-scaling resolution plane is given in figure 3.3.

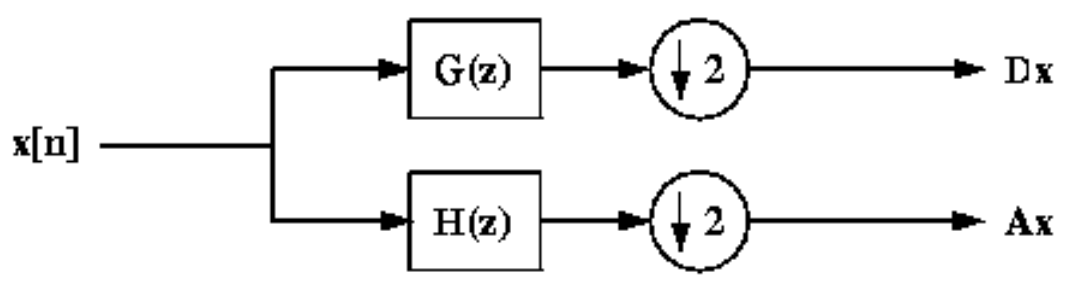

(a)

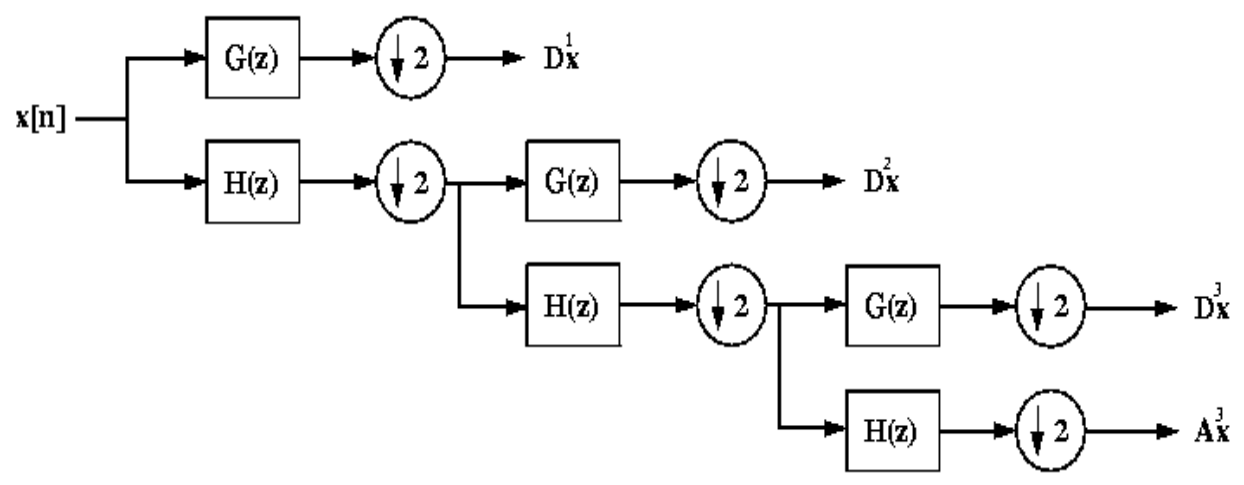

(b)

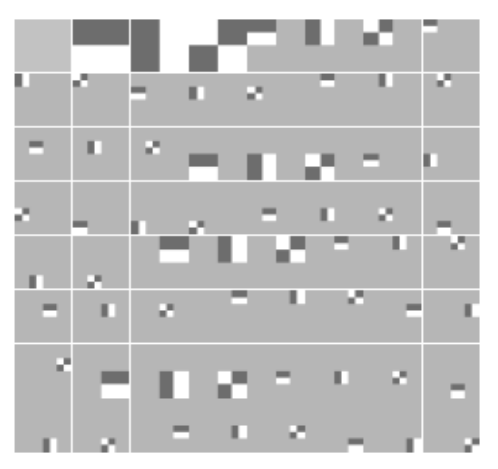

(c)

Figure 3.3. (a) DWT having one decomposition level [23] (b) DWT having three decomposition levels [23] and (c) Haar Dictionary basis [22] 


\subsubsection{Discrete Wavelet Transform}

Pure Discrete transforms are used in signal coding applications due to its computational efficiency. It is the modified version of DC-CWT where both the time and time scale parameters are discrete. Computation structure is same as DC-CWT. Simple 1D DWT having only one level is represented in Figure 3.3. (a)

Multi-scale reconstruction of the image from transformed coefficients is done using Inverse transform. Orthonormal filter method is used to design filters for IDWT. Shift variance property of DWT motivates the need for complex extended DWT. DTCWT, introduced by Kingsbury [18], was used with filters and that resulted in good shift invariance, directional selectivity and reduced over completeness. Complex filters are applied separately to rows and columns of an image which produces six bandpass bands at each decomposition level that are aligned at \pm 15 , \pm 45 and \pm 75 degrees. Complex filters help in interpreting one wavelet as the real part and the other wavelet as the imaginary part of complex-valued 2D wavelet. This complex nature provides approximate shift invariance perpendicular to wavelet orientation. These properties can be seen in Figure 3.4. DTCWT is vaguely represented as the union of four real orthonormal bases of two DTCWT trees, though DTCWT is not actually the union of four orthonormal bases. Reconstruction of the image is more accurate as the complex filters are chosen bi-orthogonal set.

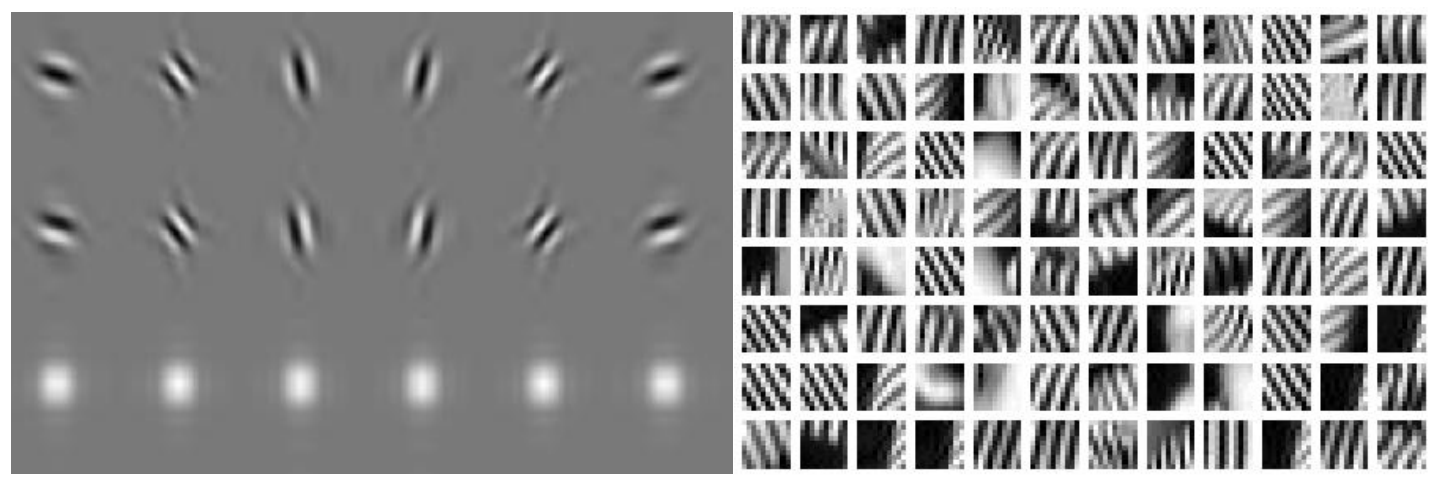

Figure 3.4. Left image representing the Wavelets associated with the orientation of DTCWT and Right image represents the Overcomplete DTCWT dictionary 


\subsection{Dictionary Learning}

The prominence of dictionary learning has grown for constructing a dictionary to well approximate a given signal. Since empirically learned dictionary can faithfully represent the signals, they can be well used for better data fusion. Suppose a training set is generated by an unknown model, it is not simple to identify the generating model and more importantly the dictionary D itself. This was first addressed by Field and Olshausen in [19]. Motivated by this, the researchers were able to develop a localized, oriented, bandpass receptive fields through dictionary learning, which are similar to the population of simple cells in visual cortex. Later the contributions by various other experts [16] [20] [21], resulted in the development of novel dictionary learning algorithms. We have presented two training methods: 1) Methods of optimal direction algorithm by Engan et al., [21] and 2) K-SVD by Aharon et al., [16].

Consider the following problem which shows that the sparse representation $x_{i}$ of signal $b_{i}$ over an unknown dictionary $D$. Assuming the error $\epsilon$ to be known,

$\min _{D,\{x\}_{i=1}^{M}} \sum_{i=1}^{M}\left\|x_{i}\right\|_{0}$ subject to $\left\|b_{i}-D x_{i}\right\|_{2}<\epsilon, 1 \leq i \leq M$

Objective of this problem is to find best representation and unknown dictionary. If the above defined problem [24] is solved, proper representations $k_{0}$ and the generating model can be found. This problem can also be presented by reversing roles of the penalty and constraints as:

$\min _{D,\{x\}_{i=1}^{M}} \sum_{i=1}^{M}\left\|b_{i}-D x_{i}\right\|_{2}^{2}$ subject to $\left\|x_{i}\right\|_{0}<k_{0}, 1 \leq i \leq M$

\subsubsection{Method of Optimal Directions Algorithm}

This is a frame design algorithm which aims to find true dictionary $\mathrm{D}$ and best representation of the source signal. We can view the two problems (3.12) and (3.13) as nested minimization problems. Objective of inner minimization is to obtain few non-zeros in the vector $x_{i}$, for a fixed dictionary D. Algorithm can be summarized as:

1) Update the dictionary in $k^{\text {th }}$ stage which is $D_{k}$ using the dictionary $D_{k-1}$ from previous stage. 
2) Using the dictionary $D_{k-1}$ solve $M$ instances of $l_{0}^{e}$, one for each data set $b_{i}$.

Solving $D_{k}$ can be done using least squares and error is evaluated using Forbenius norm:

$D_{k}=\arg \min _{D}\left\|B-D X_{k}\right\|_{F}^{2}$

$D_{k}=B X_{k}^{+}$

Table 3.1. Pseudo algorithm of MOD dictionary learning strategy [21]

Objective: To find the dictionary $\mathrm{D}$ that sparsely represents the training vector $\left\{b_{i}\right\}_{i=1}^{M}$.

Initialization: $k=0$

1) Build an initial dictionary $D_{0} \in R^{n \times m}$ by using random entries.

2) Normalize the columns of the initially constructed dictionary.

Main Iteration: Increment $k$ by 1 , then follow the steps

Sparse approximation stage: Use any efficient pursuit algorithm to find the best approximation $\hat{x}_{i}=\arg \min _{x}\left\|y_{i}-D_{k-1} x\right\|_{2}^{2}$ subject to $\left\|x_{i}\right\|_{0}<k_{0}, 1 \leq i \leq M$.

These sparsely representations for the matrix $X_{k}$.

Dictionary update stage: Update the dictionary using, $D_{k}=\arg \min _{D}\left\|B-D X_{k}\right\|_{F}^{2}$ and $D_{k}=B X_{k}^{+}$

Stopping criterion: If change in $\left\|B-D X_{k}\right\|_{F}^{2}$ is small enough, stop the process. Otherwise, follow the iteration.

Output: Dictionary $D_{k}$.

\subsubsection{K-SVD Algorithm}

Even though the objective of KSVD and MOD algorithms are same, difference lies in the dictionary update stage. KSVD algorithm developed by Aharon generalizes the K-means method for efficient dictionary learning and the atoms in the dictionary are updated sequentially. All the 
columns in the dictionary are fixed except $i_{0}{ }^{\text {th }}$ and $d_{i_{0}}$. Updating the atoms can be done, along with the coefficients that multiply them, in matrix $X$. Modifying (3.13) by isolating the dependency on $d_{i_{0}}$ as,

$\left\|B-D X_{k}\right\|_{2}^{2}=\left\|B-\sum_{i=1}^{M} d_{i} x_{i}^{T}\right\|_{2}^{2}=\left\|\left(B-\sum_{i \neq i_{0}}^{M} d_{i} x_{i}^{T}\right)-d_{i_{0}} x_{i_{0}}^{T}\right\|_{2}^{2}$

Error matrix is the term inside the parenthesis,

$E_{i_{0}}=\left(B-\sum_{i \neq i_{0}}^{M} d_{i} x_{i}^{T}\right)$

Optimal $d_{i_{0}}$ and $x_{i_{0}}^{T}$ to minimize the function is the best rank one approximation of $E_{i_{0}}$. This can be attained through Singular Value Decomposition (SVD) but this leads to a vector $x_{i}^{T}$ with more non-zero coefficients. Minimizing the term and fixing the number of non-zero coefficients can be achieved by selecting a subset of columns of $E_{i_{0}}$ whose entries that correspond to the original signal from the data samples are taken and these components use the elements of $x_{i_{0}}^{T}$ in $X$.This may lead to the variation in existing non-zero coefficients while preserving the cardinality. To remove the non-relevant columns by introducing a restriction matrix $P_{i_{0}}$ that multiplies $E_{i_{0}}$ from right, $P_{i_{0}}$ is constructed with $M$ rows (number of samples in data set) and $N$ columns (number of entries that use $j_{0}{ }^{t h}$-atom). Restriction on the row $x_{i_{0}}^{T}$ is defined as:

$\left(x_{i_{0}}^{R}\right)^{T}=x_{i_{0}}^{T} P_{i_{0}}$

This is done to choose only non-zero entries. In order to achieve optimal $d_{i}$ and optimal sparse representation $x_{i_{0}}^{R}$, a rank one approximation via SVD is applied for the sub matrix $E_{i_{0}} P_{i_{0}}$. SVD decomposition is applied for decomposing computation error by,

$E_{i_{0}}{ }^{R}=U \Delta V^{T}$

where $d_{i_{0}}$ is the first column of $U$ and $x_{i_{0}}^{R}$ is the first column of $V$ multiplied by $\Delta(1,1)$.

Table 3.2. Pseudo Algorithm of KSVD Algorithm [16]

Task: To find the dictionary $\mathrm{D}$ that sparsely represents the training vector $\left\{b_{i}\right\}_{i=1}^{M}$. 
Initialization: Initialize $k=0$

1) Build an initial dictionary $D_{0} \in R^{n \times m}$ by using random entries.

2) Normalize the columns of the initially constructed dictionary.

Main Iteration: Start the process by incrementing $k, k+=1$

Sparse approximation stage: Use any efficient pursuit algorithm to find the best approximation $\hat{x}_{i}=\arg \min _{x}\left\|y_{i}-D_{k-1} x\right\|_{2}^{2}$ subject to $\left\|x_{i}\right\|_{0}<k_{0}, 1 \leq i \leq M$.

These sparsely representations for the matrix $X_{k}$.

\section{Dictionary Update stage:}

To minimize the optimization problem, use this stage to update both the atom $d_{i_{0}}$ and obtaining the dictionary $D_{k}$, for every $i_{0}=1,2, \ldots, m$ :

1) Define the data samples that use atom $d_{i_{0}}$,

$$
\Omega_{i_{0}}=\left\{j \mid 1 \leq j \leq M, X_{k}\left[i_{0}, j\right] \neq 0\right\} .
$$

2) Compute the residual matrix and error $E_{i_{0}}=\left(B-\sum_{i \neq i_{0}}^{M} d_{i} x_{i}^{T}\right)$

Obtain $E_{i_{0}}{ }^{R}$ by restricting the pre computed error by choose only the columns corresponding to $\Omega_{i_{0}}$.

Decompose the computed representation error $E_{i_{0}}{ }^{R}$ by applying SVD decomposition

$E_{i_{0}}{ }^{R}=U \Delta V^{T}$. Update the dictionary atoms by $d_{i_{0}}=u_{1}$ and representation by $x_{i_{0}}^{R}=$ $\Delta[1,1] \cdot v_{1}$.

\section{Convergence criteria:}

$\left\|B-D_{k} X_{k}\right\|_{2}^{2} \leq \varepsilon$, otherwise apply another iteration.

Output: Dictionary $D_{k}$. 


\section{Chapter 4. Image Fusion}

Human Visual System (HVS) has an uncanny ability to visually inspect a particular scene and mentally map the focused regions in the mind. Our human visual system can perceive enormous focal volume even in complex challenging environment. Spatial alignment of the object of interest, its discrete nature and its context increases the sharpness of the human perception model. At a specified instant, human visual cortex typically perceives an object or focal volume. Among the perceived information, a few are processed and mentally mapped but other subset of information might be ambiguous and disregarded [30].

\subsection{Introduction}

Need for information fusion arose in 1950's with a search of algorithms for integrating source images from different sensors to obtain a composite image for identifying natural and manmade objects with better interpretation and classification. Image fusion can be broadly defined as the process of enhancing the perception of the scene by synergistic integration of the images of the same scene from different sensors. The single composite image should contain all the 'relevant' information with increased robustness, spatial and temporal resolution for better human and machine perception. The context of the term 'relevant' depends upon the application. While 
fusing medical images, the 'relevant' information could be the data necessary for diagnosis. Single image from a sensor cannot generate the accurate perception of the scene. Composite image from the collection of images with complementary information of the same scene from different sensors or same sensor over an extended period of time generates more description of the scene than any of the source information. For the effective registration of source images, the accuracy of co-alignment of sensors is very important. Determining the best framework for obtaining the reliable composite image that enables the effective understanding of the scene in terms of geometry and semantic interpretation is a challenging task. Benefits of fused information include reduced uncertainty, extended spatial and temporal coverage. Recognition of information fusion is not limited to clinical diagnosis but also extends to Geosciences, tracking of targets in defense systems, etc.

\subsection{Generic requirements of fusion algorithm}

The requirements for a fusion algorithm vary with the application. General requirements of the fusion algorithm for better representation of the scene are:

1) The fused image should preserve all the salient information while suppressing the noise and undesired artifacts that might mislead the observer.

2) It should be highly reliable and robust to noise and misregistration.

In order to understand the challenges that we encounter when developing information fusion algorithm, we consider two registered images of the same scene in Figure 4.1. As a result of visual inspection, the person is clearly observed in visible image, whereas some of the details like fence is not discernible. In contrast Infrared image of the same scene shows the fence details clearly but the person is imperceptible.

Challenges faced for fusing these two images are:

1) Complementary information of visible and infrared images

2) There are some common objects in both the images but of extremely opposite contrast.

3) Inputs from different sensors have different range and resolution. 
Fusion can be simply performed by averaging or adding of source images pixel by pixel. But this might produce undesired effects if the objects are of opposite contrast. Hence multi scale transform fusion scheme is proposed.

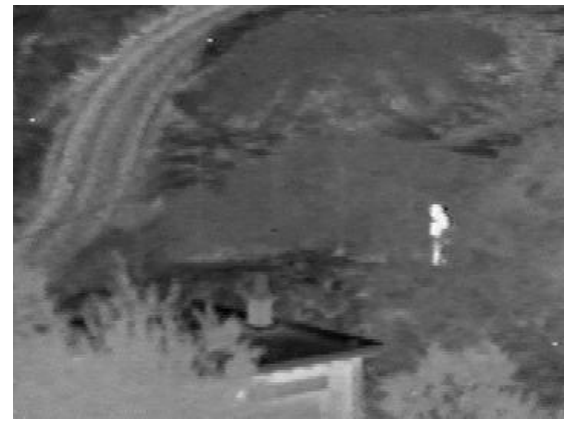

(a)

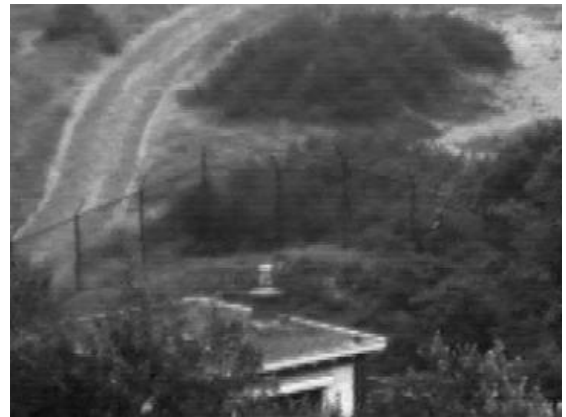

(b)

Figure 4.1. Source images to be integrated: (a) Visual image (b) infrared image.

\subsection{Preprocessing to satisfy Common Represnetation Format}

Basic requirement for fusion algorithm is observation from different sensors should have common format. Input images are compatible for fusion only when they share same representational format. Constructing a common coordinate system is the preprocessing step for image fusion algorithm. This conversion of source images to a common format includes:

1) Geometric and Temporal Alignment: Aligning the local spatial positions and local times to a common coordinate system. In our thesis, we use the already registered images.

2) Feature Extraction: This process involves the extraction of characteristic features from source images.

3) Decision Labeling: Images are transformed into multi label images by applying decision operators.

4) Semantic Equivalence: Tranformation of input images to a same object. Images should be semantically equivalent for feature extraction and decision labeling to be performed.

5) Radiometric Calibration: Transformation of input images to a common radiometric scale. It's impossible to fuse images having different radiometric calibration acquired at different illuminations. 
For decades, proper registering of images has been a progressive research area to make the different source images speak a common language for further processing. The process of aligning the multiple images of an ensemble geometrically is image registration. Basically features of the reference image will be fixed and all the source images are aligned with respect to common ground control point [25]. In earlier days, only the translational differences between the source images were registered. Introduction of corner detection [28] using gradient to register the images and introduction of segmentation to register images using optimal boundaries [26] and approximating methods [27] facilitates the registration process to align rotational and translational differences. Finding the acurrate transformation map that maps all the points from fixed image to the image to be registered is the appropriate goal for effective registration.

Quality of image registration can be analysed by: 1) Transformation model: A linear model cannot be used to register images related by non-linear transformation because that might detoriate the registration process and 2) Precision measurement by determining the landmark points: Locating the landmark points accurately and the use of efficient geometric transformation model leads to a well registered image. In [29], projective transformations are used to find the geometric relation between the images. This algorithm is constructed for the images to be registered are capturted from different angle and field of view.

\subsection{Selection methodology}

Straight forward fusion techniques like arithmetic, image gradient method, etc introduce demerits like contrast reduction in the resultant image. A good selection fusion methodology should have noise resilience property, fused image with better contrast and should preserve all the detailed information acclaiming high computational efficiency. Based on the mode of operation, fusion methods are classifies as shown below [31][32]:

(1) Pixel level fusion: Pixel by pixel operation is a straight forward fusion that can be performed in spatial domain or tranform domain. Spatial domain methods like Principal Component Analysis (PCA), weighted average method, etc integrate pixels using activity level measurement in a linear or non-linear fashion. In transform domain fusion methods like Pyramid decomposition methods, Wavelet 
Transformation method, etc. tranformed images are combined according to salience measure. Our research uses pixel level fusion method in sparse domain.

(2) Region based methods: An image is segmented into number of regions and taking the property of these regions into account, fusion rules are applied. Resultant fused image is composed of focussed regions.

\subsection{A brief review on area of application}

This thesis applies fusion in the area of Medical Computed Tomography. The objective of Computed Tomography [33] is to discern and visualize objects inside the human body noninvasively. Algorithms proposed in this thesis are specifically designed for CT in clinical applications since the issues to be considered in medical imaging and other image processing fields are different. As the non-invasive Computed Tomography (CT) is a high dose application, the increased radiation exposure is associated with the elevated risk of malignancy. This increases the need for reducing the dose but low dose might lead to noise which might hinder clinical decision making and the diagnostic process. A major area of research is devoted for processing the low dose $\mathrm{CT}$ images to interpret the scene better.

\subsubsection{Historical Milestones of Computed Tomography}

Computed Tomography is a standard imaging modality, which has revolutionized the medical diagnosis. CT is the first non-invasive method for acquiring cross sectional "slices" of anatomy to understand the internal structures of the object. Beauty of this non-invasive modality cannot be understood without the knowledge of X-rays, data processing and measurement and instrumentation [34]. Looking back through the centuries, in 400 BC, a Greek philosopher Democritus speculated the nature of matter as structure of invisible and indivisible particles. In $600 \mathrm{BC}$, another Greek philosopher Thales observed that amber acquires property of attracting even light objects when rubbed with fur. The term 'electron' meaning amber was used since amber acquired charge on rubbing.

The phenomenon of Faradays Electro Magnetic Induction in 1831 led Maxwell to discover that an accelerated charge is the source of electromagnetic radiation in 1867. According to Maxwell, electromagnetic waves which are transverse in nature can propagate through free space without 
any material medium. Hertz in 1888 demonstrated the existence of electromagnetic waves experimentally. In different regions of wavelength, electromagnetic waves were produced by varying the excitation. The most important event which has revolutionized the field of imaging is the discovery of X-rays.

In 1895, a German scientist named William Roentgen discovered when accelerated electrons strike an anode target having high atomic weight, melting point and thermal conductivity gives up kinetic energy and thereby produces electromagnetic rays. The physical properties of electromagnetic waves are determined by the wavelength. Due to the unknown nature of these rays, Roentgen called them X-rays. X-rays are shorter wavelength electromagnetic waves of range $5 \dot{A}$ to $10 \dot{A}$. These contributions are exploited by Coolidge and that led to the invention of first rotating anode x-ray tube. First CT scanner based on a radioactive source conceived by Hounsfield [36] and mathematical solutions to the Tomographic reconstruction problem by Cormack [35] are some of the major breakthroughs in the development of CT. In a very short span, CT has progressively grown to a great extent that it no longer requires optical reconstruction techniques.

\subsubsection{Working of CT}

Ability of conventional X-ray radiography is limited to obtaining 2D projections for 3D structures which results in the reduction of spatial information due to the process of averaging [37]. Averaging produces low contrast results which are difficult to interpret even for an expert. The need for eliminating the processing of averaging led to the development of Tomography which has the ability to produce radiographic slices of region of interest. This is also referred to as Tomosynthesis, if digital post processing is required. In conventional CT, X-ray tube and film are synchronously moved in opposite directions.

The point above and below the slice are blurred and points along the center of rotation are imaged sharply. Blurring angle determines the quality region of interest. Unlike conventional radiography, computed tomography avoids the superimposition of blurred structures. This results in higher contrast that enhances even the soft tissue. Four generations of CT scanners, scanners with cone shaped X-ray beam, acquisition through slip ring technology and many other advancements have been developed and this huge success is due to the leap in medical imaging 
diagnosis. Clinical applications of CT have drastically increased after the subsequent growth of helical scanning in 1980s and multi detector row technology in 1990s [43].

X-ray tube rotates perpendicularly to the length of axis of the stationary patient in a circular orbit during data acquisition. The detector arrays are opposite to the X-ray tube that absorbs the $\mathrm{x}$-ray photons from the region of interest. The Tomogram is produced after measuring the attenuation coefficients. Arrangement of x-ray tube and receptor array varies in different generation scanners. Resultant image quality and dosage are interrelated.

\subsubsection{Reconstruction methods}

Attenuation coefficient decides the intensity of the projection image. The word image refers to reconstructed $2 \mathrm{D}$ slice. The interpretation of attenuation coefficient in the object has to be computed first. Reconstruction of internal structure of object from the measured projection can be done through several ways. A matrix of $\mu$-values for the slice is constructed. These reconstruction algorithms [38] are customized based on projections at different angles and are used to find the $\mu$-values in each voxel of the slice perpendicular to the rotation axis. In early days, algebraic methods were carried out for reconstructing CT images. Since they are complex and computational intensive, computationally efficient filtered back projection methodology is used in all the modern CT systems.

\subsubsection{Computed Tomography Dose}

According to the National Council on Radiation Protection \& Measurements, the usage of CT in Medical Imaging has increased more than six fold from 1980s to 2006. Approximately, around 67 million CT examinations were made in 2006 in USA alone, which was around 3 million in 1980 [39]. This rapid increase in usage of CT is due to its fast scanning speed and isotropic spatial resolution of $0.3 \mathrm{~mm}$ to $0.4 \mathrm{~mm}$ which enables physicians to diagnose precisely in less time by providing invaluable information than other imaging modalities. But the potential risk of developing radiation induced cancer is associated with the increase of ionizing radiation exposure. Hence reducing the radiation dose in Computed Tomography as reasonably achievable as possible is a must to increase the benefit/risk ratio. 


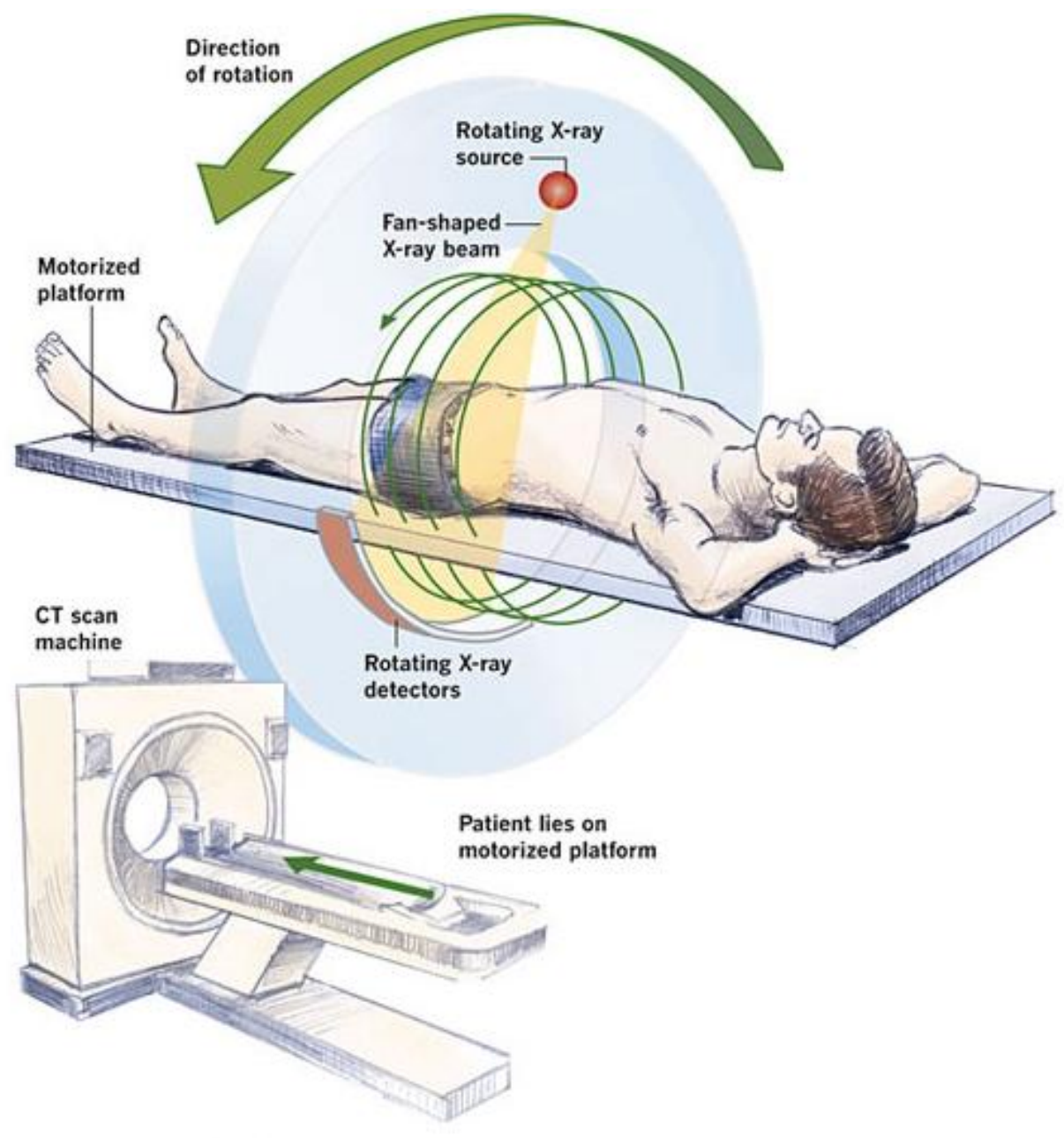

Figure 4.2. Principle of Computed Tomography [44].

Quantification of radiation dose in CT can be done using several methods such as scanner radiation output, organ dose and effective dose for evaluating the risk of developing induced malignancy. Volume CT dose index $C T D I_{v o l}$ is a standard way of representing scanner output level. These quantitative measures [41] do not directly represent the patient dosage rather they represent the scanner output.

The accuracy of $C T D I_{v o l}$ is doubtful for cone beam CT scanners. Organ dose is used to measure the radiation risk association with patients' organs according to age and sex specifications. Energy dose $D$ is the ratio of absorbed energy to structure mass and its unit is Gray. 


$$
D=\frac{\text { Energy Absorbed }}{\text { Mass of the corresponding tissue }}
$$

Since the weighting factor of different organ with respect to radiation varies, equivalent dose $D_{e q}$ of specific tissue is expressed as:

$D_{e q}=\sum_{R} W_{R} D_{T, R}$

Considering the radio sensitivity of different organs [40], effective dose represents the weighted sum of relative doses of individual organs and is expressed in $m S v$ units. Considering the tissue weighting factor, effective dose $D_{E f f e c t i v e}$ is given by:

$$
D_{\text {Effective }}=\sum_{T} W_{T} D_{e q}
$$

Quality of CT result is determined by the amount of radiation dose. Trade off between the image quality and dosage level should be understood for reducing the CT dose without compromising the image quality. Image quality is assessed by contrast-to-noise ratio (CNR) and signal to noise ratio (SNR). Dose and SNR are be related as [40]:

$S N R \propto \sqrt{\text { dose }}$

The aspects to be considered for dose reduction are: 1) Defining and understanding the expected image quality for each diagnostic task which allows high noise level and low dose without compromising the clinical diagnosis and 2) To reduce image noise that might hinder important details necessary for diagnosis. Quantum noise and electronic noise are associated with CT. Quantum noise is determined by incident radiation and photons collected by receptor. At low dosage values, quantum noise affects the quality of image. Fluctuation of electronic components in CT system results in electronic noise which degrades the image quality. Optimizing the detector and collimator in the CT system for dose performance of a CT system is very important to reduce peripheral radiation dose. Various other factors that influence the dose are tube current, exposition time, slice thickness and minimizing the detector size. Improving the reconstruction algorithm and effective data processing algorithms optimally reduce noise without sacrificing the diagnostic valuable information is important. 


\subsubsection{Computed Tomography Noise}

Visual noise and increased blurring of the image impairs the visibility of anatomic structures. To evaluate the quality of image quantitatively, signal to noise ratio is defined as the ratio of signal level to noise level.

$S N R=\frac{\mu}{\sigma}$

where $\mu$ is the mean and $\sigma$ is the standard deviation of image. Considering two registered images $I_{1}$ and $I_{2}$ to differentiate noise and structure, two disjoint subset of projections $P 1 \subset P$ and $P 2 \subset P$ having same number of samples with $P 1 \cap P 2=\varnothing$ and $P 1 \cup P 2=P$ are used to reconstruct images $I_{1}$ and $I_{2}$. Assuming the noise in $P 1$ and $P 2$ to be uncorrelated, the reconstructed image can be represented by [42]:

$I_{1}=B+N_{1}$

$I_{2}=B+N_{2}$

The ideal noise free signal, $B=E\left(I_{1}\right)=E\left(I_{2}\right)$ is assumed to have zero noise $E\left(N_{1}\right)=E\left(N_{2}\right)=$ 0 where $E(\cdot)$ is a statistical expectation operator.

Since the noise in the projections is uncorrelated, noise in corresponding reconstructed image will also be uncorrelated. Covariance of the noises of reconstructed images is given by:

$\operatorname{COV}\left(N_{1}, N_{2}\right)=0$

Local noise is estimated for each orientation to assess the difference between diagnostic detail and noise. Reconstructed of the dataset is given by:

$R=\frac{I_{1}+I_{2}}{2}$

Noise can be represented as the difference between the two datasets:

$d=I_{1}-I_{2}=N_{1}-N_{2}$

$I_{R}$ can be represented as the linear combination of random variables with weights: 
$I_{R}=I_{1} g_{1}+I_{2} g_{2}$

The reconstructed image include noise free signal, hence the variances can be expressed as:

${\sigma_{R}}^{2}={\sigma_{1}}^{2} g_{1}{ }^{2}+{\sigma_{2}}^{2} g_{2}{ }^{2}+2 g_{1} g_{2} \operatorname{COV}\left(I_{1}, I_{2}\right)$

Assuming that the noise in the projections is uncorrelated and amount of noise is approximately equal, the relation between the standard deviation in average and input data set is given by:

$\sigma_{M}=\frac{\sigma_{1}}{\sqrt{2}}=\frac{\sigma_{2}}{\sqrt{2}}=\frac{\sigma_{d}}{2}$

\subsection{Fusion Méthodologies}

\subsubsection{Image fusion using multi scale approach}

Motivated by the ability of the human visual system to analyze the information at different scales, researchers proposed the multi-scale fusion is methods. Basically, multi-scale transform fusion, like pyramid fusion, is the transformation of synergistic combination of different levels of information representation into an image mosaic. Firstly desired transformation is applied over the source images. Secondly, fusion rule is applied for transformed coefficients at different levels and each decomposition level represents different bands of image frequencies. Lastly, fused image is recovered by applying inverse transform. Framework of pyramid based fusion is shown in Figure 4.2.

Laplacian pyramid framework [45] supports the convolution of Laplacian decomposition map and Gaussian pyramid of weighting map at each decomposition level. Fusion using pyramid involves the following process:

1) The source images to be fused are decomposed to pyramid coefficients belonging to a different frequency band which are obtained as a result of low pass filtering and subsampling by a factor of 2 . 


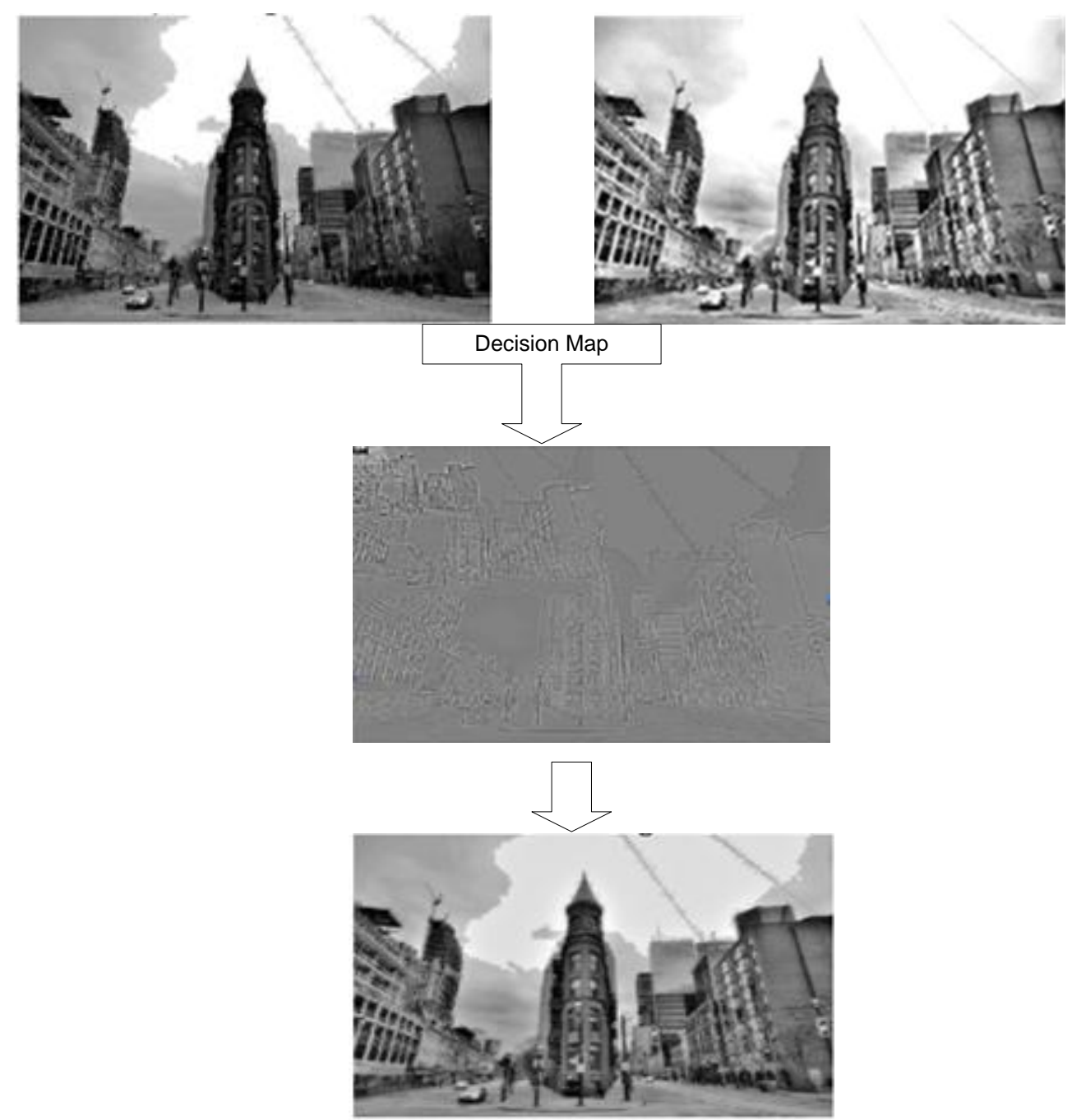

Figure 4.2. Pyramid image fusion results

2) The scalar weighting $S_{i, j, k}$ map for each pixel is calculated by combining the quality metrics contrast $C$ and the photometric measure luminance $\mathcal{L}$ whose unit is candela per square metre $\left(c d / m^{2}\right)$. This is represented by:

$S_{i, j, k}=\left(C_{i, j, k}\right)^{\alpha_{1}}\left(\mathcal{L}_{i, j, k}\right)^{\alpha_{2}}$

$\widehat{S_{l, J, k}}=\left[\sum_{k=1}^{\mathrm{N}}\left(\mathrm{C}_{i, j, k}\right)^{\alpha_{1}}\left(\mathcal{L}_{i, j, k}\right)^{\alpha_{2}}\right]^{-1}$

where $\widehat{S_{l, j, k}}$ represents the normalized weighting map, $\alpha_{1}$ and $\alpha_{2}$ are the weighting components. Then the Gaussian pyramid $G\left(\widehat{S_{l, J, k}}\right)$ of normalized weight map is calculated. 
3) Blending is performed by calculating the weighted average of Laplacian pyramid with Gaussian pyramid at each level.

$$
L\left\{I_{f}\right\}_{i, j}^{n}=\sum_{k=1}^{N} \mathrm{G}\left\{\widehat{S_{l, J, k}}\right\}_{i, j, k} L\{I\}_{i, j, k}^{n}
$$

4) Finally, reconstruct the fused image by expanding and adding all the levels of Laplacian pyramid $L\left\{I_{f}\right\}_{i, j}^{n}$.

Redundancy between different scales in pyramid decomposition method and unavailability of directional information are the major limitations of this method. Wavelet based approach has benefits over pyramid based approach. In the following section multi-scale wavelet transform fusion has been discussed.

\subsubsection{D-Discrete Wavelet Transform Fusion}

Since the image data are discrete in nature, DWT can be very well used for image processing tasks. Moreover there exists a dependency between spatial resolution and frequency. One decomposition results in four frequency bands LL, LH, HL and HH. Second decomposition will be applied over the current LL band. This is shown in Figure 4.3. When DWT [53] is applied over the image to be processed, the low frequency bands will have low spatial resolution and high frequency bands will have high spatial resolution.

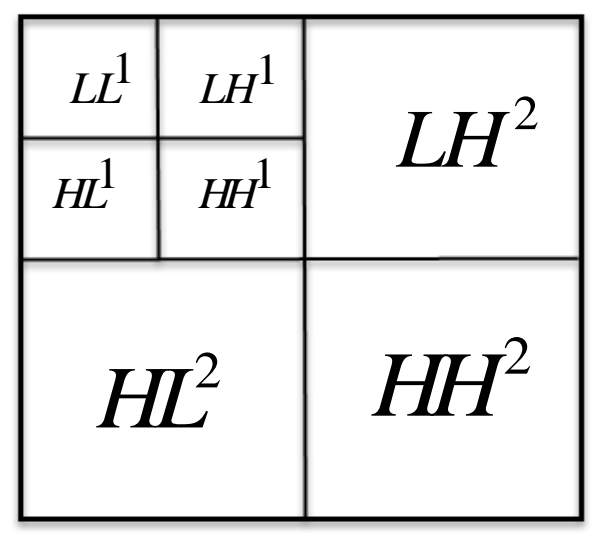

\section{1,2---Decomposition levels}

$\mathrm{H}$---High frequency bands

L---Low frequency bands

Figure.4.3. 2D Decomposition; level=3 


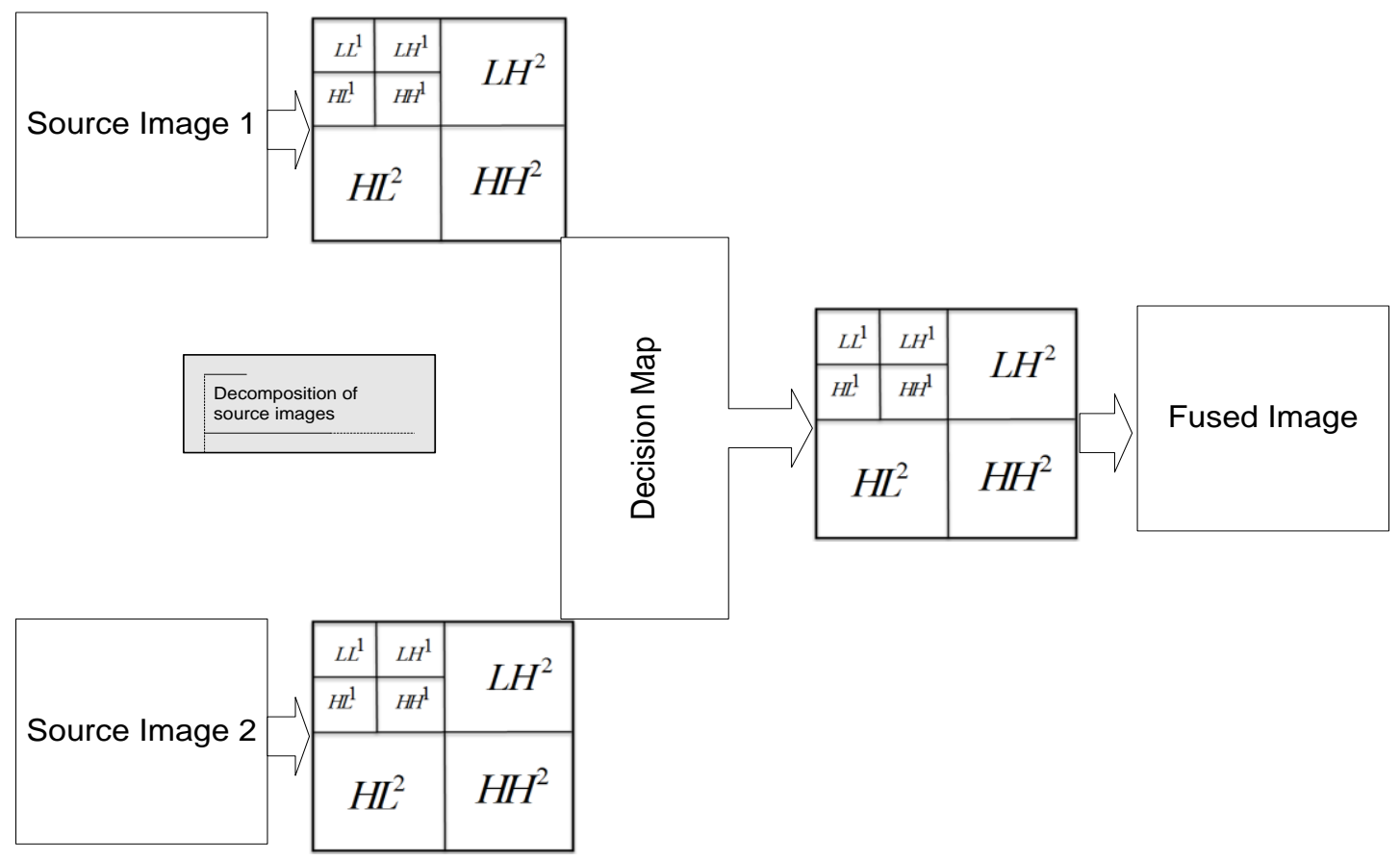

Figure.4.4. 2D DWT Fusion framework

In LL band, the spatial resolution will be very low and it shows the approximate coefficients. In contrary, diagonally opposite $\mathrm{HH}$ band shows the detailed coefficients and has higher spatial resolution.

Following are the steps to fuse images using DWT:

1) Images to be fused are decomposed using wavelet transform.

2) Fusion decision map is constructed by choosing the appropriate transformed coefficients that is represented better by any of the source images in different decomposition levels. Pixel (maximum selection or weighted average) or Window based fusion rule can be applied to fuse the transformed coefficients. This process is shown in Figure 4.4.

3) Fused image can be recovered by applying inverse transform. Recovered image $I_{f}$ can be reprseneted by:

$I_{f}=T^{-1}\left(\phi\left(T\left(I_{1}\right), T\left(I_{2}\right) \ldots, T\left(I_{n}\right)\right)\right.$ 
Work flow of DTCWT Fusion [51] is presented in Figure 4.5. First, the Source images to be fused are decomposed into detailed and approximate coefficients using DTCWT. Fusion rule is applied to form decision map. Fused image $I_{f}$ is recovered by applying inverse transform.

$$
I_{f}=T^{-1}\left(\phi\left(T\left(I_{1}\right), T\left(I_{2}\right) \ldots, T\left(I_{n}\right)\right)\right.
$$

$I_{1}$

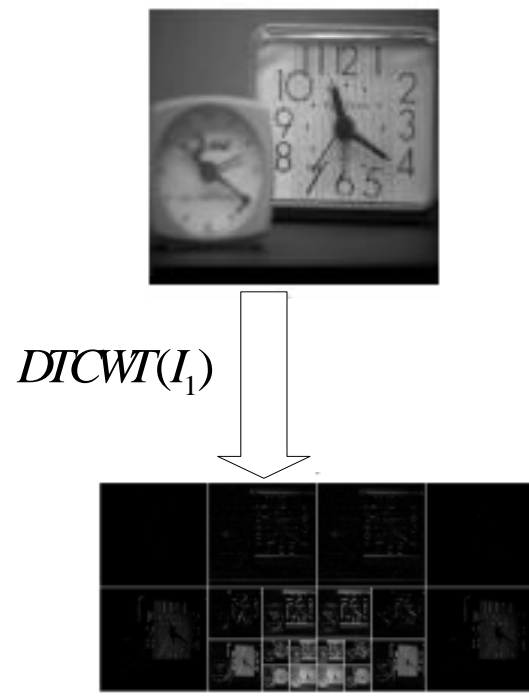

$I_{2}$

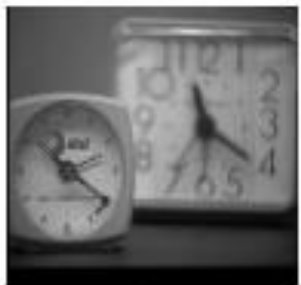

$\operatorname{DTCWT}\left(I_{2}\right)$

Decision Map
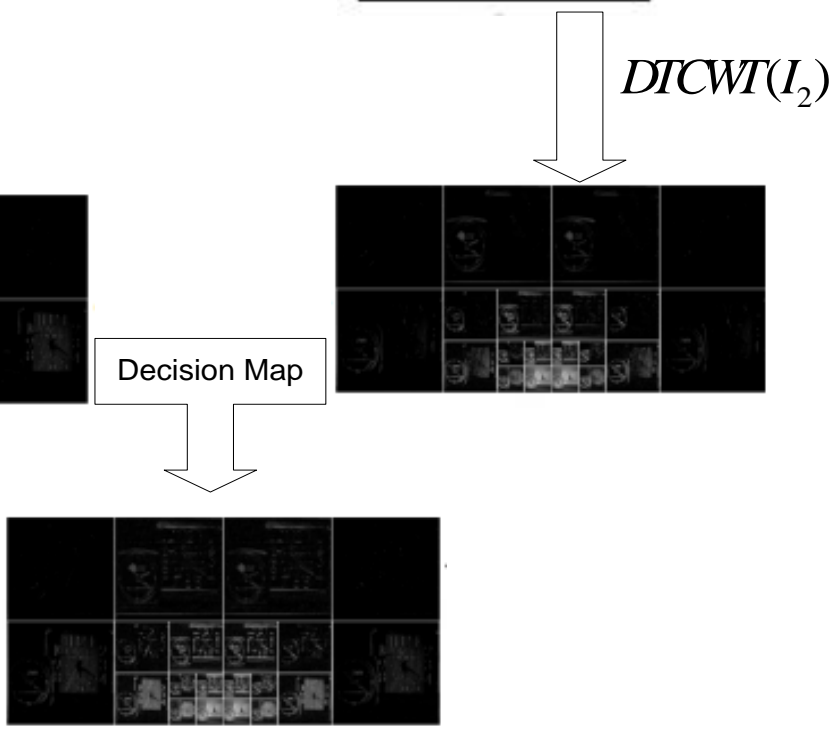

IDTCWT

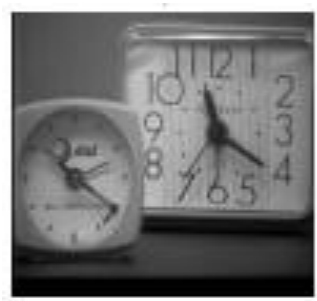

Figure.4.5. 2D DTCWT Fusion framework

\subsubsection{Principal Component Analysis based fusion}


This unsupervised dimension reduction technique is based on the idea of projecting the high dimensional information into a low dimensional sub-space. Specifically, this technique is carried out by constructing the covariance matrix from the input images [47] [46].

To understand this best known linear sub-space transformation technique, we consider an image $I$ having $M$ rows and $N$ columns as a one dimensional vector $b$. Finding the low dimensional subspace in which the input image resides from the $M N$ dimensional space is the basic idea of this statistical technique. If $A=\left(a_{1}, a_{1}, \ldots, a_{j}\right)$ is a $M N \times j$ array that represents a set of $i$ orthonormal basis functions, the transformation into a single dimensional vector of reduced length $L$ can be represented as follows:

$\tilde{b}=A^{T}(b-\phi)$

where $\phi$ is a $M N \times 1$ column vector and $\tilde{b}$ has the length $L \leq M N$. Approximation of $b$ can be reconstructed from $\tilde{b}$ by applying inverse transform as:

$\hat{b}=A^{T}(\tilde{b}+\phi)$

Error obtained from recovered vector $\hat{b}$ and the input vector $b$ should be minimal.

To adequately represent the source images as the linear combination of orthogonal basis, we seek the orthonormal vectors $A=\left(a_{1}, a_{2}, \ldots, a_{j}\right)$ with $j \ll M N$. For a given training set of $K$ images represented by $b_{K}$ one dimensional vectors, directions are selected along the training vectors having largest variances. The dimension is reduced to $L$ by discarding the directions having minimal variance.

Lower dimensional representation enhances the generalization and the visualization. Hence it provides better understanding of essentialities in the information. Manipulations performed in low dimensional space is computationally efficient than manipulating high dimensional data since the latter requires more memory and consumes more time.

Considering the spatially and temporally aligned source images to be fused $I_{1}$ and $I_{2}$ of size $M \times N$, the orthonormal vectors are $A=\left(a_{1}, a_{2}, \ldots, a_{j}\right)$. Columns vectors of source images can 
be represented as $b_{1}$ and $b_{2}$. The orthonormal vectors are the Eigen vectors of covariance matrix $C_{v}$.

$C_{v}=\frac{1}{2} \sum_{K=1}^{2}\left(b_{K}-\phi\right)\left(b_{K}-\phi\right)^{T}$

where $\phi=\frac{1}{2} \sum_{K=1}^{2}\left(b_{K}\right)$

$C_{v} a_{j}=\lambda_{j} a_{j}$

If $a_{j}$ is of length $M N$, then the covariance matrix $C_{v}$ will have the dimension of $M N \times M N$. If there are many source images, then Eigen vector decomposition will become computationally tedious. Eigen vector can also be calculated using an alternative algorithm known as TurkPentland algorithm. Eigen vector can be calculated by initially constructing $Y$ using column vectors $b_{1}$ and $b_{2}$ as:

$Y=\left(\left(b_{1}-\phi\right),\left(b_{2}-\phi\right)\right)$

Let $e_{j}$ be the $j^{\text {th }}$ eigenvector of covariance matrix $C_{v}=Y^{T} Y$,

$Y^{T} Y e_{j}=\lambda_{j} e_{j}$

$Y Y^{T} Y e_{j}=C_{v} Y e_{j}=\lambda_{j} Y e_{j}$

Hence $a_{j}=Y e_{j}$ is the Eigen vector of $C_{v}=Y^{T} Y$. For the unitary matrix $A$, the eigen spaces are orthogonal. We select a small subset to find a low dimensional sub-space that yields better performance. We know that using covariance matrix, PCA is performed. For improving signal to noise ratio, correlation matrix can be used in place of covariance matrix.

$\rho_{j}=\frac{C_{v}}{\sigma_{j}}$

In order to equalize the dynamic range of $a_{j}$, eigen vectors are divided by $\sqrt{\lambda_{j}}$. Whitening is the combination of PCA and normalization. This normalization can be represented mathematically as: 
$\frac{1}{\sqrt{\Lambda}}=\left(\begin{array}{ccc}\frac{1}{\sqrt{\lambda_{1}}} & \cdots & 0 \\ \vdots & \ddots & \vdots \\ 0 & \cdots & \frac{1}{\sqrt{\lambda_{M N}}}\end{array}\right)$

Corresponding whitening vector is:

$\tilde{b}_{W}=\frac{1}{\sqrt{\Lambda}} A^{T}(b-\phi)$

Applying the above equation, covariance matrix becomes unit matrix. This shows that the whitening vector is invariant to further orthonormal transformations and the whitening vector can be rotated for maximum discriminate power.

By converting the two dimensional data to a column vector, row to row relationships between pixel gray levels is destroyed. Performing statistical operations directly on input images instead of column vector preserves row to row relationships. This is the main idea of 2D-PCA. Normalizing each source images $A_{K}$,

$A_{N_{K}}=A_{K}-\Psi$

Covariance matrix can be constructed by,

$C_{v}=\frac{1}{n} \sum_{K=1}^{n} A_{N_{K}}{ }^{T} A_{N_{K}}, K \in\{1,2, \ldots, n\}$

Using weighted superposition, source images are fused together. As discussed earlier, PCA converts the input image into its Eigen space by selecting the principal components having influencing Eigen values, thereby preserving the salient areas and enhancing the spatial resolution of the source images without the introduction of artifacts and hence signal to noise ratio is improved.

Following steps are involved in fusion using PCA

Step 1: covariance matrix $C_{v_{N}}$ is calculated for $\mathrm{N}$ source images. Converting the two dimensional source images to one dimensional column vector, covariance matrix can be written as:

$C=\operatorname{cov}\left(I_{f}\right)=\operatorname{cov}\left(\left[I_{1}(:), I_{2}(:), \ldots, I_{N}(:)\right]\right)=\frac{1}{N-1} I_{f} \cdot I_{f}^{*}$ 
where $I_{f}$ is the fused image.

Step 2: Eigen space of the covariance matrix can be calculated by:

$\left[E_{v}, E\right]=e i g(C)$

where $E_{v}$ is the eigenvector and $E$ is the influencing Eigen value

Step 3: Eigen vector can be normalized by:

$E_{v}(:, i)=\frac{E_{v}(:, i)}{\operatorname{sum}\left(E_{v}(:, i)\right)}, \quad i=1, \ldots, N$

Step 4: Normalized Eigen vector of maximum Eigen value is selected. Final fused image is reconstructed by considering these Eigen vectors as weightings.

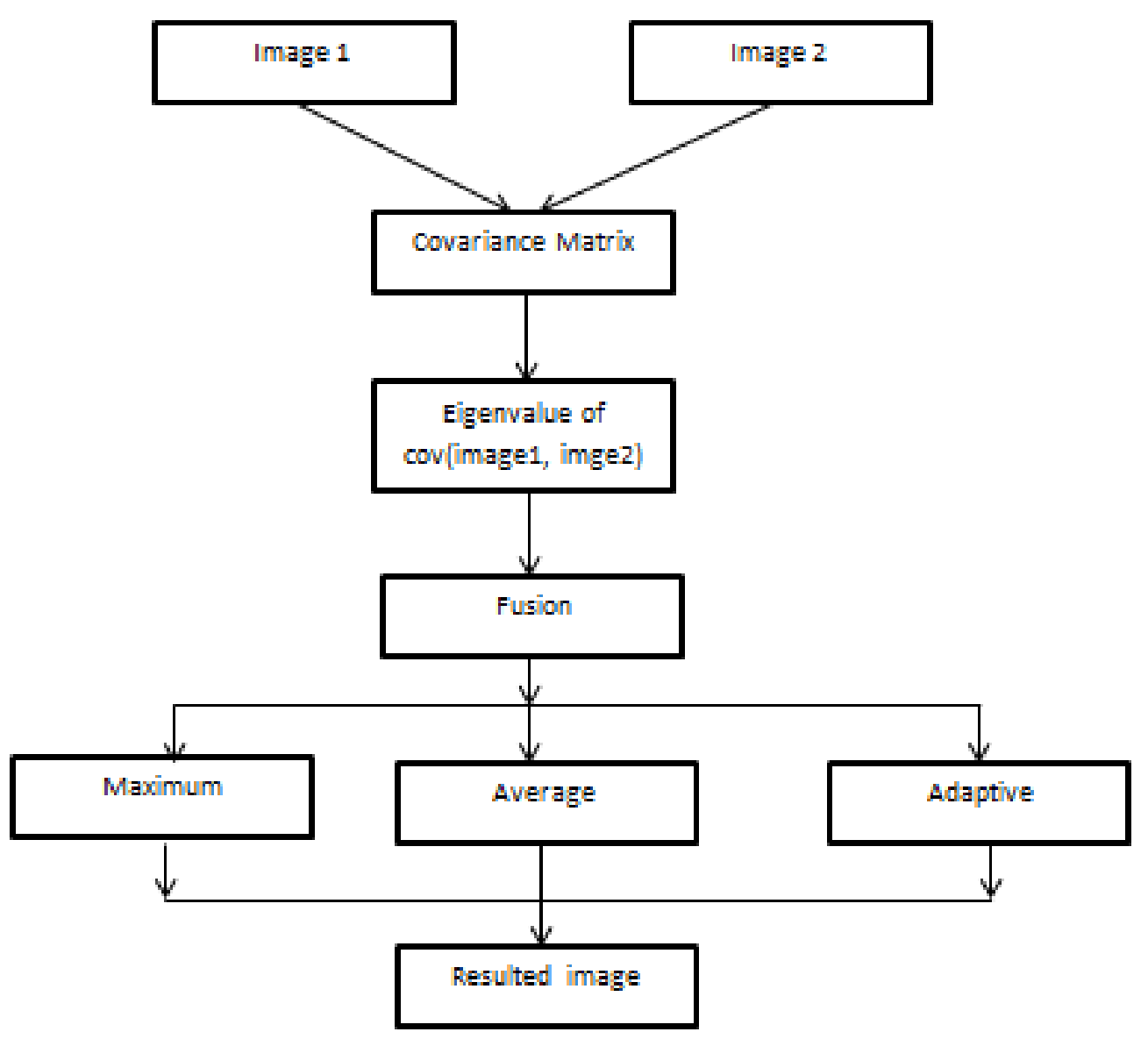

Figure.4.6. PCA Fusion framework 


\subsection{Image Quality Measurement}

Assessing the performance of a fusion result is done based on quantitative and qualitative measures. Qualitative analysis is the measure of observers' experience and can be done by quantifying the subjective evaluation done by experts. Measuring the quality of image mathematically without any human observer is image quality metric. There are mainly two different ways of quantitatively measuring the quality of fused image based on spatial and spectral similarity: 1) Non-reference method which states the quality of the fused image without requiring any reference. This is very challenging due to the lack of ground truth. 2) Reference method which requires both reference and composite image for IQ metric evaluation. Brief reviews on some of the metrics which are used in thesis are presented as follows:

Entropy: It measures the amount of details in the image; the high entropy indicates high details. Since the first order entropy doesn't consider the spatial correlation, therefore we employed the second order entropy as quality evaluation which will enable us to measure the transition between gray levels. The second order entropy is based on the co-occurrence matrix $t_{i, j}$ and its probability $p_{i, j}$ as given bellow [45][54],

$H=-\sum_{i} \sum_{j} p_{i, j} \log \left(p_{i, j}\right)$

where $p_{i, j}=\frac{t_{i, j}}{\sum_{k=0}^{L-1} \sum_{l=0}^{L-1} t_{l, k}}$

Absolute mean brightness error (AMBE) [45]: It measures the shift in mean intensity of the fused image $m_{f}$ from mean intensity of the original image $m_{o}$ and it is given as [54],

$A M B E=\left|m_{o}-m_{f}\right|$

\section{Mutual information [54]:}

To find the mutual information of registered image $I_{1}$ and reference image $I_{r}$,

$$
M I\left(I_{1}, I_{r}\right)=\iint p_{I_{1}, I_{r}}(a, b) \log _{2} \frac{p_{I_{1}, I_{r}}(a, b)}{p_{I_{1}}(a), p_{I_{r}}(b)}
$$


where $p_{I_{1}, I_{r}}(a, b)$ is the joint probability a pixel $(\mathrm{x}, \mathrm{y})$ in $\mathrm{I}_{1}$ has a gray-level a and the same pixel in B has a gray level b.

Normalized mutual information $\operatorname{NMI}\left(I_{1}, I_{r}\right)$ may vary if the number of pixels which are common to the source images changes. Even a small variation may lead to inaccuracies in this spatial alignment. So normalized mutual information can be used:

$$
N M I\left(I_{1}, I_{r}\right)=\left\{\begin{array}{l}
\frac{M I\left(I_{1}, I_{r}\right)}{-\iint p_{I_{1}}(a) \log _{2} p_{I_{1}}(a) d x d y-\iint p_{I_{r}}(b) \log _{2} p_{I_{r}}(b) d x d y} \\
\frac{M I\left(I_{1}, I_{r}\right)}{\min \left(-\iint p_{I_{1}}(a) \log _{2} p_{I_{1}}(a) d x d y,-\iint p_{I_{r}}(b) \log _{2} p_{I_{r}}(b) d x d y\right)} \\
\frac{M I\left(I_{1}, I_{r}\right)}{-\iint p_{I_{1}, I_{r}}(a, b) \log _{2} p_{I_{1}, I_{r}}(a, b) d x d y} \\
\frac{M I\left(I_{1}, I_{r}\right)}{\sqrt{\iint p_{I_{1}}(a) \log _{2} p_{I_{1}}(a) d x d y \cdot \iint p_{I_{r}}(b) \log _{2} p_{I_{r}}(b) d x d y}}
\end{array}\right.
$$

PSNR [54]: It is Peak Signal to Noise Ration which is used to measure the reconstruction quality of fused image. PSNR of the fused image $\mathrm{I}_{\mathrm{f}}$ is calculated using the standard formula:

$$
\operatorname{PSNR}\left(I_{f}\right)=10 \log _{10}\left(\frac{M^{2}}{M S E}\right)
$$

where $\mathrm{M}$ is the maximum possible pixel value of the image and MSE is the Mean Square error. While the SNR and RMSE are given as follows, where $r(x, y)$ is the reference image and $t(x, y)$ is the fused image .

$$
\begin{aligned}
& S N R=10 \log _{10}\left[\frac{\sum_{x=0}^{n x-1} \sum_{y=0}^{n y-1} r(x, y)^{2}}{\sum_{x=0}^{n x-1} \sum_{y=0}^{n y-1}[r(x, y)-t(x, y)]^{2}}\right] \\
& R M S E=\sqrt{\frac{1}{n x n y} \sum_{x=0}^{n x-1} \sum_{y=0}^{n y-1}[r(x, y)-t(x, y)]^{2}}
\end{aligned}
$$


SSIM [55]: It is the Structure Similarity Index which provides structural information of objects in the image. This measure is based on similarity of contrast of local patch $c(i, j)$, structure of local patch $s(i, j)$ and luminance of local patch $l(i, j)$. PSNR estimate perceived errors where as SSIM assumes image degradation as the perceived change in structural information. Structural information considers the spatially close pixels having strong inter-dependencies that carry the salient details. Range of SSIM is between -1 and 1 , and value 1 occurs only for identical data set.

\section{Contrast to Noise Ratio (CNR)}

There is a tradeoff between radiation quality and contrast and effect or influence of this can be described using Contrast to Noise Ratio (CNR) [56]. CNR has certain properties which are given below:

1) Relates to contrast difference between larger object and background of the image.

2) Relates to exposure factors.

3) Doesn't incorporate any information about resolution. 


\section{Chapter 5. Proposed Methodologies}

\subsection{Image Fusion Using Simultaneous Controlled St-OMP}

The goal of integrating the source images using sparse representation method is to enhance the important details without the introduction of distortion. Signal can effectively be represented by sparse representation as the linear combination of effective sparse model and well designed dictionary. Salient features of the images can be represented with least number of coefficients using sparse representation which is effective than traditional multi transform methods because of the nature of sparsity and overcompleteness of dictionary. Sparsity results in many zero coefficients thereby leading to the improvement of computational efficiency. It is not necessary for the atoms to be orthogonal in overcomplete dictionary and number of atoms are more than number of image pixels. We utilize Modified Cosine Transform Dictionary generated by cosine basis functions incorporated with shift variations. We use sparse approximation and utilize effective dictionaries to fuse low dose and medium dose $\mathrm{CT}$ images. The process of image fusion using sparse representation contains two stages: Signal approximation stage and fusion stage. In signal approximation stage, all the patches of the source images are transformed into linear combination of atoms of, MDCT dictionary tailoring matching pursuit methodology specifically designed for fusion application. Generally, the approximation solution is achieved by matching pursuit algorithms through an iterative process. Locally optimum solution is obtained in each iteration when residual vector closely resemble the vector to be approximated and global 
optimum solution is obtained after the sequence of locally optimum solutions. Due to the computational efficiency of OMP over BP, it is used to solve the optimization problem in applications. In fusion stage, global approximated solution of source images are integrated by applying absolute maximum fusion rule.

Inspired by [52], we are proposing a simultaneous approximation method which adopts CStOMP for representing the several source images as linear combination of elementary signals at once. St-OMP discussed in previous chapter that uses many atoms each stage solves the optimization problem in less iteration. St-OMP uses threshold value in the aim of selecting highest matching atoms from the well designed dictionary. So the approximation depends upon the threshold value and every atom above the threshold value contributes to reconstruct the signal. So lower threshold might allow more non-zero entries at each stage. In contrast, high threshold allows only few non-zero entries each stage to reconstruct the signal. But this takes more iteration to find the approximate solution due to the dependency on threshold value and it is computationally intense due to the calculation of pseudo inverse at each stage. In order to overcome this, CSt-OMP is proposed to approximate a single input signal at a time.

We propose Simultaneous CSt-OMP that adopts a different atom selection strategy that solves the optimization problem more efficiently and in less time. Considering a matrix $B_{F}$ of size $d \times K$ whose columns are $K$ source images, Simultaneous CSt-OMP solves the following optimization problem:

$$
\min _{C}\left\|B_{F}-D C\right\|_{2}^{2} \text { subject to }\|C\|_{0}<T
$$

where $C$ is the coefficient matrix having $T$ non zero coefficients. 


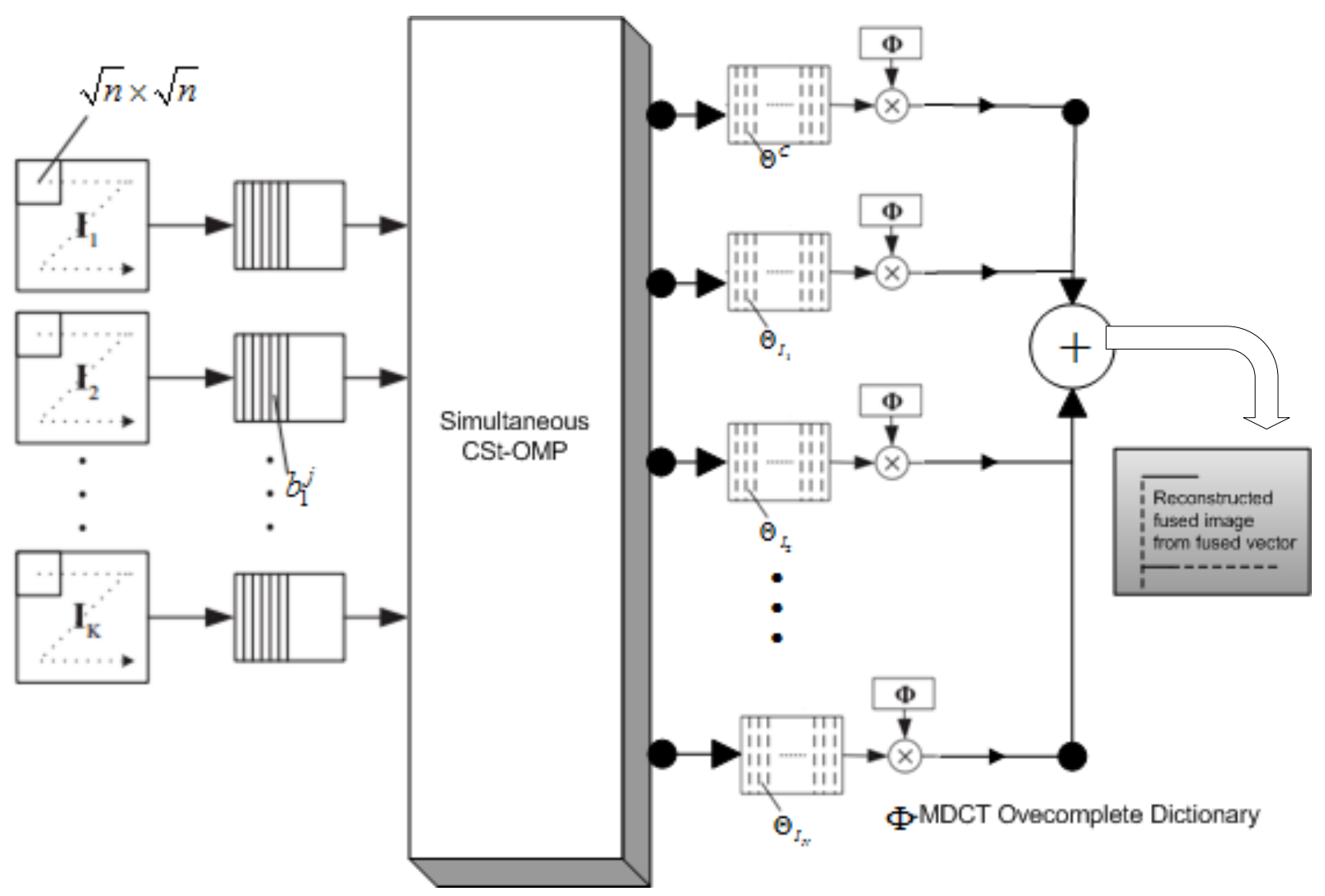

Figure 5.1. Framework of proposed Simultaneous Controlled St-OMP fusion

In this algorithm, number of atoms to be added at each stage is pre-declared thereby reducing the dependency on preset threshold value. Framework of the proposed method is shown in figure 5.1. The residual matrix starts out to be the corresponding training signals itself. The objective of each iteration is to minimize the optimal simultaneous representation error at each stage.

Hard thresholding selects the matching atoms when the inner product of row vectors of dictionary and residual is maximum and these selected atoms are stored in a local dictionary $D_{\text {Local }}$ to be updated. In the seletion strategy, largest matching atom is selected. Covariance of largest matching atom with $D_{\text {Local }}$ is computed and the least correlated atom is chosen as the second representative since it contributes more on signal reconstruction. The process should be repeated unless the reconstruct error is optimally minimum. Due to the less dependency on threshold value, at the same time using two atoms in each stage and approximating simultaneously makes this algorithm efficient than SOMP algorithm. 
Objective: Construct an approximation for the problem $\mathrm{P}_{0}$

$\left(\mathbf{P}_{\mathbf{0}}\right): \min \|C\|_{0}$ subject to $S_{F}=D C$ and fuse the approximated coefficients

User defined input parameters: Dictionary $\mathrm{D}$, Signal matrix $S_{F}$ for $K$ source images and error threshold $\epsilon_{0}$.

\section{Sparse coding stage}

Initial conditions: $\mathrm{k}=0$, start by setting

Initialize residual matrix $r^{0}=S_{F}-D C^{0}$ (set initial solution $C^{0}=0$ )

Initial index set $S^{0}=\varnothing$

Iteration process: $\mathrm{k}=\mathrm{k}++$ (incrementing)

Sweep stage: Calculate the correlation $C$ of residual $r^{k}$ and dictionary D.

Hard Thresholding stage: $\{i \mid C(i)>$ Thershold $\}$ and store the atoms in local dictionary $D_{\text {Local }}$.

Update solution stage: If the inner product between row vectors $d_{k}$ of local dictionary $D_{\text {Local }}$ and residual is $d_{i}$ and is maximum, it is the most relevant first atom. Calculate the covariance between maximum matching and $D_{\text {Local }}$, then select the least representative as second atom.

Update the solution: Obtain the approximation $C_{k}$, by $\left(\left(D_{k}{ }^{T} D_{k}\right)^{-1} D_{k}{ }^{T} S_{F}\right)$ the solution to $\left\|S_{F}-D C\right\|_{2}^{2}$

Stopping condition: check the stopping criterion. If $r^{k}<\epsilon_{0}$, global simultaneous optimum solution is obtained; Otherwise, proceed the iteration process again.

Approximation stage output: $C^{k}$ is the global optimum solution obtained after $k$ iterations.

\section{Fusion stage}

$$
C_{f}^{k}=\phi\left(c_{1}^{k}, c_{2}^{k}\right)
$$


Output: Reconstructed final fused image $I_{F}$.

We assume the source images to be spatially aligned. If the source images are not registered, proper registration algorithms should be used to align the image spatially and temporally in order to avoid geometric distortion. Consider $N$ geometrically registered source images. The fusion process is given below:

1) Source image $I_{n}$ is divided into $\sqrt{n} \times \sqrt{n}$ possible patches using sliding window technique and then all the patches are transformed into $n$-dimensional column vector.

2) Using CSt-OMP, find the sparse approximation of corresponding vectors.

3) Fusion rule to be applied

Choosing the appropriate fusion rule determines the quality of final fused image. There are two important concerns to be addressed for fusion using pixel level algorithms 1) Activity level measurement identifies the salient coefficients of the source images. 2) Coefficient combination uses the salient coefficients from the source images in to the fused image. Weighted average fusion scheme smoothens the salient information. Thus maximum absolute fusion rule is applied to preserve the salient information.

$$
\begin{aligned}
C_{f}^{k} & =\phi\left(c_{1}^{k}, c_{2}^{k}\right) \\
C_{f}^{k} & =\arg \max _{i=1,2, \ldots, N}\left(\left|c_{i}^{k}\right|\right)
\end{aligned}
$$

4) Fused vector $V_{f}^{k}$ is calculated by:

$$
V_{f}^{k}=D C_{f}^{k}
$$

5) Final fused image is recovered from $V_{f}^{k}$ vector by reshaping this to block size of $\sqrt{n} \times \sqrt{n}$. Each pixel position of the fused image $I_{F}$ is the summation of several block values. Final image is obtained by dividing each pixel of the final image by number of summations occurred at that pixel.

6) The overcompleteness nature of dictionary and the sliding window technique makes this algorithm shift invariant. 


\subsection{Medical Image Fusion Based on Joint Sparse Method}

Image fusion using sparse representation methodology guarantees robustness which can extract the underlying information from source images effectively. As we have seen in previous chapters, sparse representation techniques are used to represent signals with the fewest number of non-zero coefficients.

For a signal $B_{i}$, sparse representation assumes the representation of the signal as a linear combination of given atoms from the overcomplete dictionary $D \in \mathbb{C}^{n \times m}$ where $(n \ll m)$. Overcomplete dictionary has the capability to sparsify the signal efficiently since number of atoms exceeds number image pixels. Every signal can be represented as $b=D x$, where $x \in \mathbb{C}^{m}$ is the sparse vector that contain few salient entries. Since dictionary has more number of columns than rows, $b=D x$ is an under-determined system and there can be infinite solutions. Finding the approximate solution for the signal is not easy due to the strict equality constraint. Hence, the following optimization problem is solved to find the best approximation:

$$
\operatorname{argmin}_{x}\|x\|_{0} \text { subject to }\|D x-b\|_{2}<\epsilon
$$

where $\|x\|_{0}$ represents the number of non zero coefficients which can be positive or negative in $x$ and $\epsilon$ is the representation error. Since the above defined optimization problem is NP-hard, greedy algorithms are used to solve due to the computational efficiency and less intense nature. Non-zero sparse coefficients of the source images are considered to be the salient features for fusion. In this section the problem of fusion and denoising low dose CT images can be simultaneously addressed. Low dose and medium dose CT images of the same region of interest possess their own characteristics and also share common information. Sparse coefficient vectors are estimated for representing the ensemble of source images to be fused using the matching pursuit methodology. Proposed algorithm effectively extracts the common and complementary information and integrates effectively.

This thesis proposes a new algorithm inspired by joint sparse representation model for compressed sensing [62], which employs different rules to extract common and innovative 
sparse components from the source images. Inspired by [62] [48] and [60], a novel image fusion algorithm is proposed which integrates the source vectors in sparse domain using Principal Component Analysis.

Low dose and medium dose images of the same region of interest to be fused form an ensemble. Sparse theory states that any signal can be sparsely represented. In the same way, the ensemble of signals are represented by $S_{i}$ where $i \in 1,2, \ldots \ldots, N$ will also have joint sparse nature. That means all the data in an ensemble $S_{i}$ will have a common sparse component and each image will have innovative sparse components. Assuming the sparse basis $\Psi$ for $\mathbb{C}^{m}$ is known where $S_{i}$ is approximately represented. For the signal $i$, let the measurement matrix be $D_{i}$ of size $n_{i} \times K$. And the measurement matrix will have different entries for different $i$.

Joint sparse model I: Sparse common component plus many innovative components

In this model, all the source signals will have common components and each signal will have an innovative component and this can be represented as:

$$
S_{i}=s_{c}+s_{i}, i \in\{1,2, \ldots, N\}
$$

where $s_{c}=\Psi \Theta_{C}$ and $s_{i}=\Psi \Theta_{I_{i}}$. This means the $s_{C}$ is the common component of $S_{i}$ and $\left\|\Theta_{C}\right\|_{0}=K$ in basis $\Psi$. Whereas $s_{i}$ are the unique proportions of ensemble having $\left\|\Theta_{I_{i}}\right\|_{0}=K_{i}$ in the same basis $\Psi$. We use joint sparse model I for our experiment.

Joint sparse model II: Common sparse model

In this model, all the signals which are taken over an extended period of time are constructed from same basis $\Psi$ but having different coefficients $\Theta_{i}$ :

$$
S_{i}=\Psi \Theta_{i}, i \in\{1,2, \ldots, N\}
$$

Thus all the signals can be represented sparsely with $K$ non-zero coefficients using different coefficient in the same basis having Kelements.

For a signal $B_{i} \in \mathbb{C}^{n \times L}$, there is a common component $\Theta_{C} \in \mathbb{C}^{K \times L}$ and $i$ innovative components $\Theta_{I_{i}} \in \mathbb{C}^{K \times L}$. Signal $B_{i}$ can be represented as: 


$$
B_{i}=B_{C}+B_{I_{i}}=D \Theta_{C}+D \Theta_{I_{i}}+n_{i}
$$

Where $D \in \mathbb{C}^{n \times K}(n<K)$ is the overcomplete dictionary and $n_{i}$ is the noise component.

Innovative components might contain noise. The above equation can be sparsely represented by:

$$
\left(\begin{array}{c}
B_{1} \\
\vdots \\
B_{n}
\end{array}\right)=\left(\begin{array}{cccc}
D & D & \cdots & 0 \\
D & 0 & \cdots & 0 \\
\vdots & \vdots & \ddots & \vdots \\
D & 0 & \cdots & D
\end{array}\right)\left(\begin{array}{c}
\Theta_{C} \\
\Theta_{I_{1}} \\
\vdots \\
\Theta_{I_{n}}
\end{array}\right)+\left(\begin{array}{c}
n_{1} \\
n_{2} \\
\vdots \\
n_{n}
\end{array}\right)
$$

This can be generally written as:

$$
B=D \Theta+n
$$

if

$$
B=\left(\begin{array}{c}
B_{1} \\
\vdots \\
B_{n}
\end{array}\right), D=\left(\begin{array}{cccc}
D & D & \cdots & 0 \\
D & 0 & \cdots & 0 \\
\vdots & \vdots & \ddots & \vdots \\
D & 0 & \cdots & D
\end{array}\right), \Theta=\left(\begin{array}{c}
\Theta_{C} \\
\Theta_{I_{1}} \\
\vdots \\
\Theta_{I_{n}}
\end{array}\right) \text { and } n=\left(\begin{array}{c}
n_{1} \\
n_{2} \\
\vdots \\
n_{n}
\end{array}\right)
$$

Extracting the $\Theta_{I_{i}} \in \mathbb{C}^{K \times L}$ among the source images is the most challenging task.

Sparse vector can be calculated using any of the sparse approximation techniques for solving the following optimization problem,

$$
P_{0}: \min _{\Theta}\|\Theta\|_{0} \text { subject to }\|B-D \Theta\|_{2} \leq \varepsilon
$$

In our proposed method, we apply simultaneous controlled stage wise orthogonal matching pursuit proposed in the previous section to solve it. Selection of efficient dictionary plays an important role in extracting the salient features from the source images. As we know the limitations of fixed dictionaries, we use dictionary learning technique to learn the basis vectors that are capable of well representing the signal. 
The source images are traversed from left top to right bottom using sliding window technique and divide the image into $j$ number of $\sqrt{n} \times \sqrt{n}$ patches. These patches are then converted lexiographically into $j$ column vectors for each image $b_{j_{i}} \in \mathbb{C}^{n}$. As discussed all the source images are constructed using the same subset of atoms in the dictionary. Sparse coefficients are considered as image features where the coefficient of each patch is calculated separately by solving the optimization problem $P_{0}$ under the overcomplete dictionary $D$. The signal in the $j^{\text {th }}$ ensemble can be represented as:

$$
b_{j_{i}}=D \Theta_{C}+D \Theta_{I_{i}}
$$

Where $\Theta_{C}$ and $\Theta_{I i}$ denotes the sparse coefficients of common and innovative information and JSM can be represented as:

$$
B_{j}=D \Theta_{j}
$$

where $B_{j}$ is the ensemble assuming the signals are noiseless and

$$
\begin{aligned}
& B_{j}=\left[\left(b_{j_{1}}\right)^{T},\left(b_{j_{2}}\right)^{T}, \ldots \ldots,\left(b_{j_{N}}\right)^{T}\right] \in \mathbb{C}^{N n}, \text { Dictionary } D=\left[\begin{array}{ccc}
D & \cdots & 0 \\
\vdots & \ddots & \vdots \\
D & \cdots & D
\end{array}\right] \in \mathbb{C}^{N n \times(N+1) K} \text { and } \\
& \Theta_{j}=\left[\left(\Theta_{C}\right)^{T},\left(\Theta_{I_{1}}\right)^{T}, \ldots \ldots,\left(\Theta_{I_{N}}\right)^{T}\right] \in \mathbb{C}^{(N+1) K}
\end{aligned}
$$

But ensemble of low dose and medium dose images can be represented as:

$$
B_{j}=D \Theta_{j}+n
$$

where $n$ is the noise. $\Theta_{j}$ can be obtained by solving the optimization problem using the Simulataneous CSt-OMP method with appropriate error tolerance. The detailed steps of the algorithm are summarized:

1) We assume the source images $I_{1}, I_{2} \in \mathbb{C}^{N \times M}$ have common representation format. All the images to be integrated are divided into $\sqrt{n} \times \sqrt{n}$ patches using sliding window technique and are lexicographically transformed to $n$-dimensional column vectors. Each source image will have $(N-\sqrt{n}+1) \times(M-\sqrt{n}+1)$ patches. 
2) Mean values $m_{i}^{j}$ of $(N-\sqrt{n}+1) \times(M-\sqrt{n}+1)$ patches of the source images is subrtracted from the patches and can be represented as:

$$
\bar{b}_{i}^{j}=b_{i}^{j}-m_{i}^{j}
$$

where $\bar{b}_{i}^{j}$ is the joint representation of a common component $\bar{b}_{c}^{j}$ and two innovative components $\bar{b}_{1}^{j}, \bar{b}_{2}^{j}$ and can be calculated by solving the optimization problem.

3) Appropriate fusion rules are applied to integrate the sparse vectors. Fused innovative sparse vector $s_{j_{i}}{ }^{F}$ is obtained by integrating innovative sparse vectors using PCA rule.

Using PCA, orthogonal directions enhancing the variance of the innovative sparse vectors is obtained. Unlike other fusion rules in sparse domain, PCA preserves salient features while eliminating the artifacts since innovative components might have noise. PCA converts sparse vector into its Eigen space which is done by selecting principal component having influencing Eigen values. Covariance matrix of sparse vectors is calculated as:

$$
s_{c}^{j}=\operatorname{cov}\left(\left[\bar{b}_{1}^{j}(:), \bar{b}_{2}^{j}(:)\right]\right)
$$

Then the Eigen space of the covariance matrix is calculated by:

$$
\left[b_{V}^{j}, b_{D}^{j}\right]=\operatorname{eig}\left(s_{c}^{j}\right)
$$

where $b_{V}^{j}$ is the Eigen vector and $b_{D}^{j}$ is the Eigen value. Normalized Eigen vector of maximum Eigen value is chosen as the weightings of the images to be fused.

$$
\overline{b_{V}^{J}(:, l)}=\frac{b_{V}^{j}(:, i)}{\operatorname{sum}\left(b_{V}^{j}(:, i)\right)}, \quad i=1, \ldots, N
$$

Innovative fused vector is obtained by:

$$
b_{f}^{j}=\overline{b_{V}^{J}(:, 1)} \bar{b}_{1}^{j}+\overline{b_{V}^{J}(:, 2)} \bar{b}_{2}^{j}+\cdots+\overline{b_{V}^{J}(:, N)} \bar{b}_{N}^{j}
$$

4) Fused innovative vector $s_{f}^{j}$ and sparse vector of common component are fused using "choose-max" rule since the common components are noiseless, that is the vector with 
maximum $l_{0}$ norm is considered as the salient feature. Weighted average fusion rule is used to fuse the mean values.

$$
s_{m}^{j}=\Phi\left(s_{f}^{j}, s_{C}^{j}\right)
$$

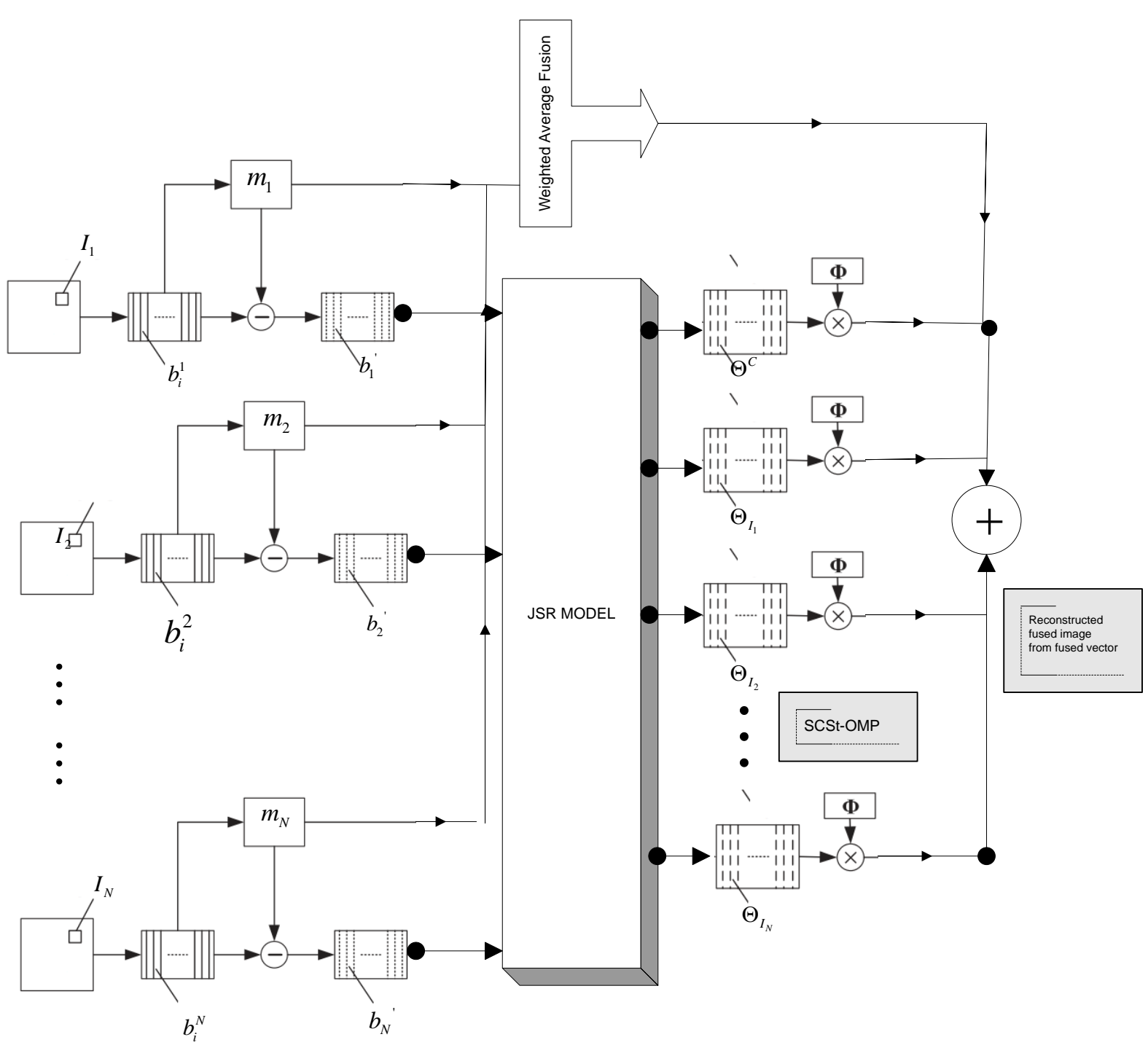

Figure 5.2. Framework of Joint Sparse PCA fusion methodology

Weighted average fusion of mean values can be done by:

$$
m_{f}^{j}=\Phi\left(m_{1}^{j}, m_{2}^{j}\right)=\mathrm{T} m_{1}^{j}+(1-\mathrm{T}) m_{2}^{j}
$$


where the weighting parameter $T=\frac{1}{\left(1+\exp \left\{-\tau\left(\left\|\bar{b}_{1}^{j}\right\|_{2}-\left\|\bar{b}_{2}^{j}\right\|_{2}\right)\right\}\right)}, \tau>0$

5) Final fused vector is calculated by combining with the overcomplete dictionary:

$$
b_{F}^{j}=D s_{f}^{j}+D s_{m}^{j}+m_{f}^{j}
$$

Lastly, $b_{F}^{j}$ is reshaped into a block of size $\sqrt{n} \times \sqrt{n}$. All the blocks are appropriately placed in the specific location of the fused image. Many block values represent single pixel value due to the usage of sliding window technique and hence final fused image is obtained by average processing at each pixel location.

Table 5.2. Pseudo algorithm of Joint Sparse PCA fusion method

Objective: To integrate the source images

User defined input parameters: Dictionary $\mathrm{D}, N$ source images mage $I_{N}$ and error threshold $\epsilon_{0}$.

Initial conditions: $\mathrm{k}=0$, start by setting

Initialize residual matrix: $r^{0}=S_{F}-D C^{0}\left(\right.$ set initial solution $\left.C^{0}=0\right)$

Initial index set: $S^{0}=\varnothing$

Iteration process: $\mathrm{k}=\mathrm{k}++$ (incrementing)

\section{Sparse coding stage:}

-Common and innovative vectors through JSR

-Patches of the original images are subtracted from the mean of patches $\bar{b}_{i}^{j}=b_{i}^{j}-m_{i}^{j}$

-Stopping condition: check the stopping criterion. If $r^{k}<\epsilon_{0}$, global simultaneous optimum solution $s_{c}^{j}$ is obtained; Otherwise, proceed the iteration process again. 


\section{Fusion stage:}

-PCA fusion to integrate innovative vectors $s_{c}^{j}=\operatorname{cov}\left(\left[s_{1}^{j}(:), s_{2}^{j}(:)\right]\right)$

-weighted average rule to fuse $b_{c}^{j}$ and common component $s_{m}^{j}=\Phi\left(s_{f}^{j}, s_{C}^{j}\right)$

-weighted average of mean values $s_{m}^{j}=\Phi\left(s_{f}^{j}, s_{C}^{j}\right)$

Output: Reconstructed final fused image $I_{F}$.

\subsection{Simultaneous medical image fusion and denoising using focused region in sparse domain}

To the best of our knowledge only the contributed methods in this thesis are used to fuse low dose and medium dose images and denoise them simultaneously. It is necessary to know that most of the fusion algorithms are developed based on the assumption that the images are noise free. Such algorithms are effective for noise free images and presence of noise might compromise the quality of fusion. Since the proposed hybrid Sparse PCA method seems to show promising results, a novel image fusion is proposed which uses an initial fused image from the previous method. Then in sparse domain, pixels of fused image similar to the pixels of the original image are determined and considerably focused region map is obtained. Final fused image is obtained after simultaneous dictionary learning, fusion and denoising in sparse domain. This algorithm is designed for both noisy and noise free source images.

\subsubsection{Dictionary learning and acquisition of initial fused image}

First step starts out to be dividing the source images in to $\sqrt{n} \times \sqrt{n}$ using sliding window technique and lexicographically ordered as $n$-dimensional column vectors $b_{i}^{j}$. From the previous section we know that, an ensemble of intercorrelated images has a common component and multiple innovative components. Initial fused vector $b_{F_{i}}^{j}$ is obtained by Joint Sparse PCA method that employs different fusion rules for integrating common, innovative sparse components and mean of patches. Dictionary $D$ learnt from high dose patches are used for representing the signal. Integrated image is obtained by: 


$$
b_{F_{i}}^{j}=D b_{m_{i}}^{j}+m_{f_{i}}^{j}
$$

where $b_{m_{i}}^{j}$ is the fused vector of common and innovative sparse vectors and $m_{f_{i}}^{j}$ is the fusion of mean of patches and dictionary $D$ is trained using high dose patches.

Dictionary learning method is used for joint sparse fusion method. Proposed method uses a dictionary learning method specifically for joint sparse model. Let us assume an ensemble $S_{i}$, $i \in\{1,2, \ldots, N\}$ containing low dose and medium dose images.

Mathematical definition of objective function can be written as:

$$
\operatorname{argmin}_{D, \Theta} \frac{1}{2}\left\|S_{i}-D \Theta\right\|_{F}^{2} \text { subject to }\|\Theta(\mathrm{t})\|_{0} \leq L
$$

where this function denotes sparsity, $L$ denotes the number of non zero coefficients. Optimization problem subject to certain amount of error tolerance $\varepsilon$ is give by:

$$
\underset{\Theta}{\operatorname{argmin}}\|\Theta\|_{0} \text { subject to }\left\|S_{i}-D \Theta\right\|_{2}<\varepsilon
$$

Where $D$ represenets the dictionary that governs the optimality of sparse representation. Firstly, joint sparse coding is done and $\Theta$ is obtained through proposed simultaneous controlled OMP by fixing the dictionary $D$ and the general equation can be represented as:

$$
\left(\begin{array}{c}
s_{1} \\
\vdots \\
s_{N}
\end{array}\right)=\left(\begin{array}{cccc}
D_{C} & D_{I} & \cdots & 0 \\
D_{C} & 0 & \cdots & 0 \\
\vdots & \vdots & \ddots & \vdots \\
D_{C} & 0 & \cdots & D_{I}
\end{array}\right)\left(\begin{array}{c}
\Theta_{C} \\
\Theta_{I_{1}} \\
\vdots \\
\Theta_{I_{N}}
\end{array}\right)+n
$$

Then the dictionary is updated keeping $\Theta$ fixed by solving the problem given below:

$$
\min _{D} f(D)=\min _{D} \frac{1}{2}\left\|S_{i}-D \Theta\right\|_{F}^{2}=\min _{D} \sum_{i=1}^{N} \frac{1}{2}\left\|S_{i}-D\left(\Theta_{C}+\Theta_{I_{i}}\right)\right\|_{F}^{2}
$$

This can be extensively written as 


$$
\min _{D_{C} D_{I}}\left\|\left[S_{1} \ldots . S_{N}\right]-\left[D_{C} D_{I}\right]\left[\begin{array}{ccc}
\Theta^{C} & \ldots & \Theta^{C} \\
\Theta_{I_{1}} & \ldots & \Theta_{I_{N}}
\end{array}\right]\right\|
$$

Optimum is obtained by setting the gradient function to zero:

$$
\begin{aligned}
& \frac{\mathrm{d}}{d D} f(D)=0=-\sum_{i=1}^{N}\left[S_{i}-D\left(\Theta_{C}+\Theta_{I_{i}}\right)\right]\left(\Theta_{C}+\Theta_{I_{i}}\right)^{T} \\
& =-\sum_{i=1}^{N} S_{i}\left(\Theta_{C}+\Theta_{I_{i}}\right)^{T}+\sum_{i=1}^{N} D\left(\Theta_{C}+\Theta_{I_{i}}\right)\left(\Theta_{C}+\Theta_{I_{i}}\right)^{T}
\end{aligned}
$$

For $W=-\sum_{i=1}^{N} S_{i}\left(\Theta_{C}+\Theta_{I_{i}}\right)^{T}$ and $H=\sum_{i=1}^{N}\left(\Theta_{C}+\Theta_{I_{i}}\right)\left(\Theta_{C}+\Theta_{I_{i}}\right)^{T}$

Then we yield,

$$
W=D H
$$

When rank of the matrix $\mathrm{H}$ is not $K$ then according to Land weber [61],

$$
D^{j+1}=D^{j}+\frac{1}{\sigma}\left(W-D^{j} H\right) H^{T}
$$

where noise intensity $\sigma>\left\|H^{T} H\right\|$. Iterative joint sparse coding stage change $\Theta$ and hence spectral norm has to be calculated again. A good initial point hasten the computation since Hermitian matrix's spectral norm is known for its enhanced Eigen value [61].

If SVD of $H$ is $=V \sum V^{\prime}$, dictionary is initially updated by

$$
D^{0}=W H^{\dagger}
$$

Due to the diagonal dominance theory [61], optimum of the equation (5.32) is obtained. We normalize the atoms in the dictionary $D$ so that $\left\|d_{k}\right\|_{2}=1$ and if $d_{k} \neq 0$, the corresponding coefficient is scaled but when $d_{k}=0$, any non-zero column of $S_{i}$ is substituted after the normalization of atoms. 


\subsubsection{Detection of focused region and fusion scheme}

Source vectors $b_{i}^{j}$ and initial fused vector are compared and similar points are determined. Using certain quantitative measures like RMSE and correlation, proper focused vector $b_{F \text { focussed }}^{j}$ is designed. Source image vectors, focused vector and initial fused vector are jointly represented as common and innovative components using a trained dictionary after subtracting from the mean of the corresponding patches.

$$
b_{j_{F}}=\bar{b}_{i}^{j}+\bar{b}_{F_{\text {focussed }}}^{j}+\bar{b}_{F_{i}}^{j}=D \Theta_{C}+D \Theta_{I_{i}}
$$

where $\Theta_{I_{i}}$ is obtained by applying PCA fusion for integrating innovative components

$$
\Theta_{I_{i}}=\Phi\left(\bar{\Theta}_{i_{I}}^{j}, \bar{\Theta}_{F_{\text {focussed }}}^{j}, \bar{\Theta}_{F_{i_{I}}}^{j}\right)
$$

Final fused vector is obtained by

$$
b_{F}^{j}=D b_{m}^{j}+m_{f}^{j}
$$

where $b_{m}^{j}$ is the composite vector of common and complementary components and $m_{f}^{j}$ is obtained by applying weighted average rule to fuse the mean of patches of JSR components. Finally, the final image is constructed by positioning the blocks in designated location and averaging each pixel location. 


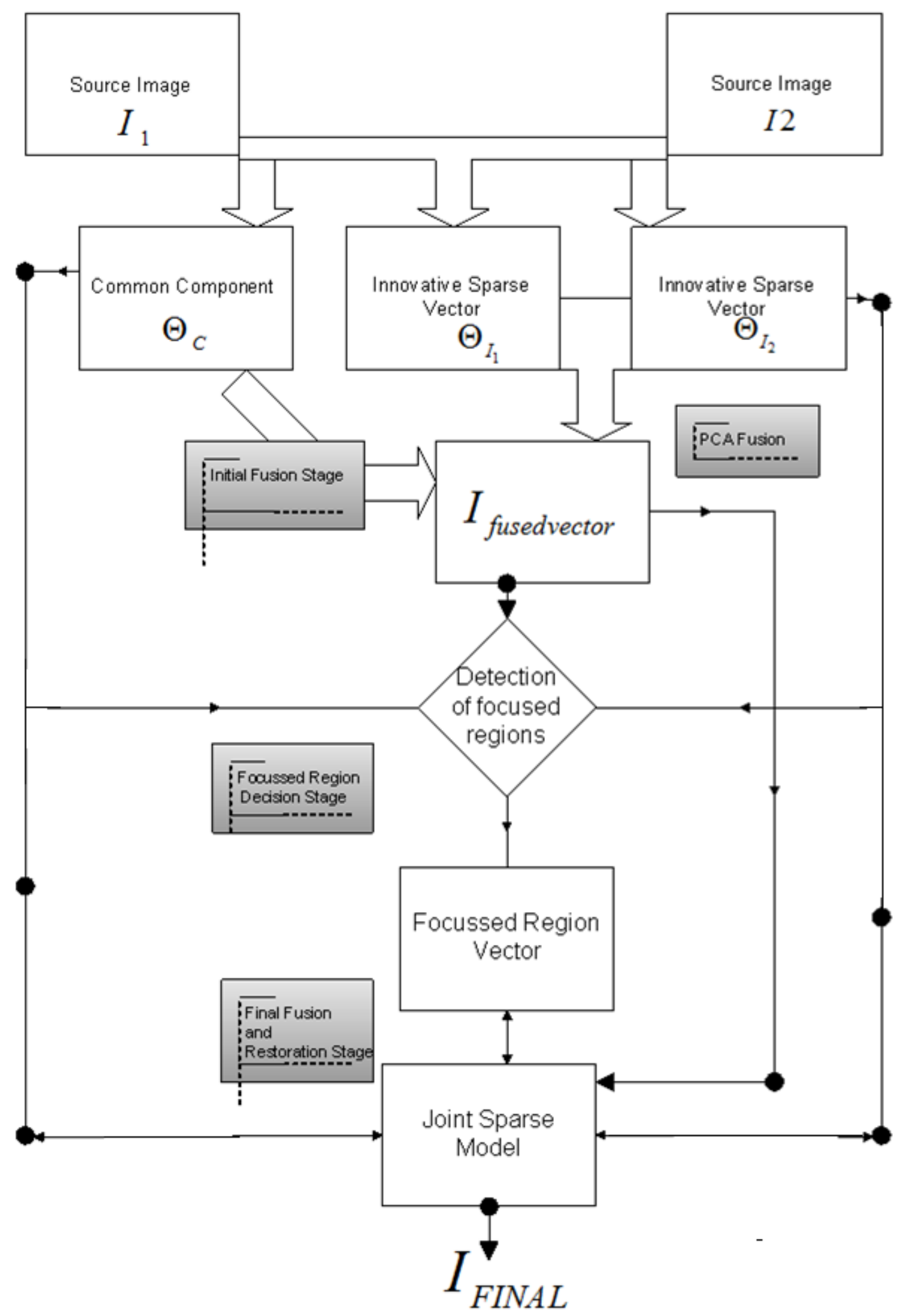

Figure5.3. Framework of fusion based on focused region 
Table 5.3. Pseudo algorithm of fusion based on focussed region

Objective: To integrate the source images

User defined input parameters: Dictionary $\mathrm{D}, N$ source images $I_{N}$ and error threshold $\epsilon_{0}$.

Initial conditions: $\mathrm{k}=0$, start by setting

Intialize residual matrix $r^{0}=S_{F}-D C^{0}\left(\right.$ set initial solution $\left.C^{0}=0\right)$

Initial index set $S^{0}=\varnothing$

Iteration process: $\mathrm{k}=\mathrm{k}++$ (incrementing)

Initial fused image stage:

objective function is solved by $\min _{D_{C} D_{I}}\left\|\left[S_{1} \ldots S_{N}\right]-\left[D_{C} D_{I}\right]\left[\begin{array}{ccc}\Theta^{C} & \ldots & \Theta^{C} \\ \Theta_{I_{1}} & \ldots & \Theta_{I_{N}}\end{array}\right]\right\|$

$\Theta^{C}, \Theta_{I_{1}}, \ldots ., \Theta_{I_{N}}$ obtained at the end of all iterations.

Initial fused image is obtained by $I_{\text {initial }}=\phi\left(\Theta^{C}, \Theta_{I_{1}}, \ldots, \Theta_{I_{N}}\right)$

Focused region detection stage: Focused vector is selected by comparing

$\Theta_{\text {initial }}, \Theta^{C}, \Theta_{I_{1}}, \ldots, \Theta_{I_{N}}$

Final fusion stage: Final fused vector is $\Theta_{\text {final }}=J \operatorname{SS}\left(\Theta_{\text {Initial }}, \Theta_{\text {focussed }}, \Theta_{1}, \ldots . ., \Theta_{N}\right)$

Stopping condition: check the stopping criterion. If $r^{k}<\epsilon_{0}$, global simultaneous optimum solution is obtained; Otherwise, proceed the iteration process again. 


\section{Chapter 6. Results and Discussion}

\subsection{Implementation using Graphical User Interface}

A graphical user interface lets the user interact with the program and makes them understand the information through graphical icons. GUI is efficient and easy to use compared to the steep learning curve of command line interfaces. GUI can free the user from understanding intricate algorithms and complex command languages. User friendliness results in transparency thereby letting the user to understand the program.

We develop GUI in Matlab environment 2013. Besides basic functions such as loading the source images and displaying the fused image, there are certain initial parameters which allow the user to assign values for dictionary size, number of sparse coefficients and number of iterations. Quantitative evaluations results are shown for evaluating the proposed methods. Button titled "input images" allows the user to load the source image to be fused. Compatible formats are gray scale images of JPEG, bmp, gif and dicom format.

Parameters for experimental setup can be initialized by adding values at the respective labeled input data box. Primary purpose of this GUI is to fuse low dose and medium dose CT images using proposed methods and to quantitatively measure the performance. Hence this algorithm is compatible to dicom images. 

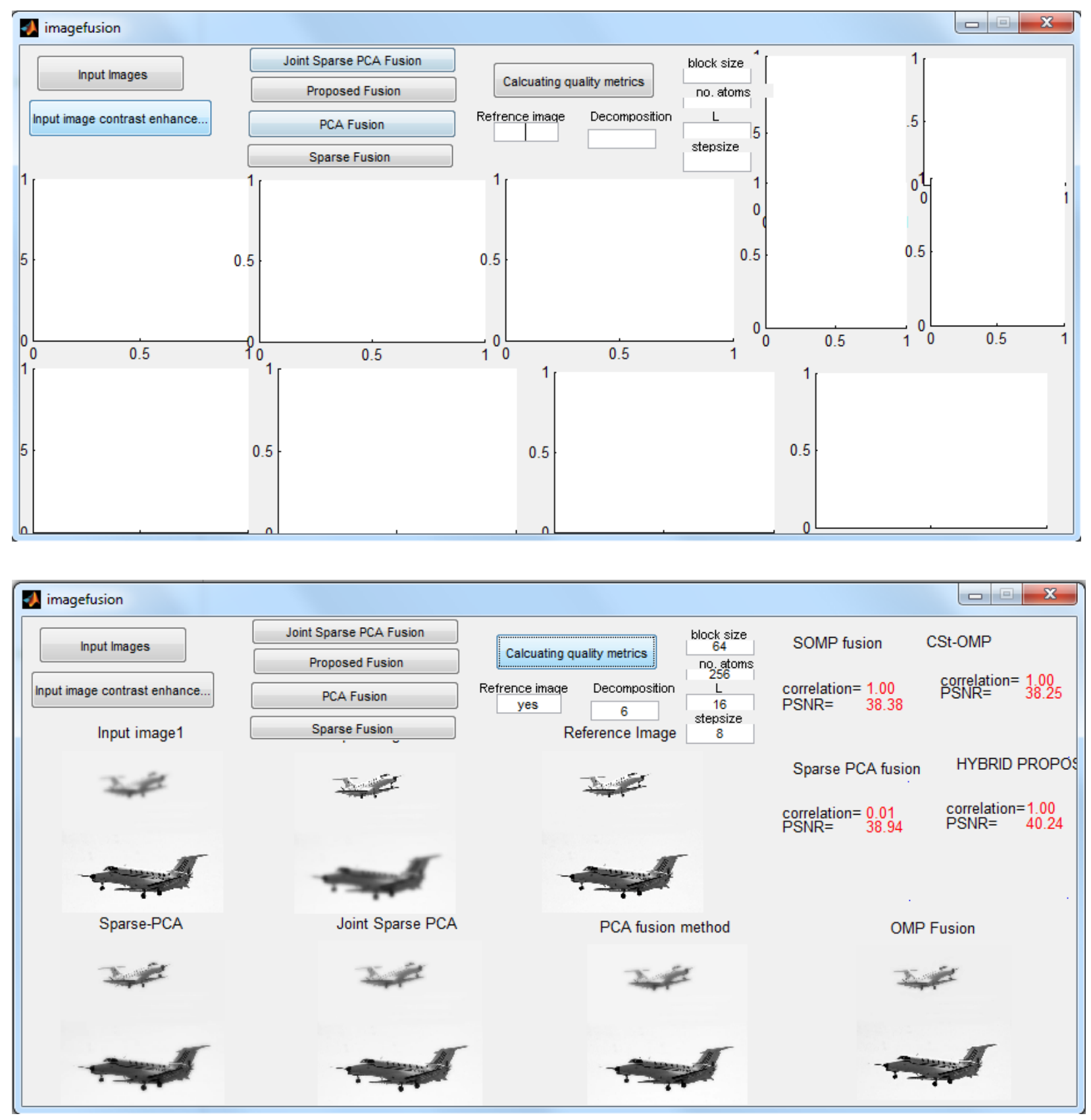

Figure 6.1. Screenshot of Graphical User Interface for pixel level fusion method

\subsection{Experimental setup and results of Simultaneous Controlled St-OMP}

To evaluate the performance of the proposed method, we use overcomplete dictionaries like MDCT dictionary and overcomplete DCT dictionary that consists of 64 non-redundant bases. 
Overcomplete dictionaries which can also contain non-orthogonal atoms, overcomes the limitations of fixed dictionary. MDCT dictionary used in our proposed method is created with 6 shifts in the first part, 4 in the middle and 2 in the last and is shown in Figure 6.6 (b). Overcomplete DCT is constructed using 29 frequency divisions from 0 to $2 \pi$ without any phase. Constructed dictionaries contain 841 atoms and are used to approximately represent the images in sparse domain using the conventional OMP fusion method and the proposed method.

Two parameters decide the efficiency of sparse coding stage: 1) Selection of appropriate size of sliding window and 2) Global representation error. We know that dictionary size is large for larger sliding window size and computational efficiency becomes lower. Decreasing the size of sliding window makes the sparse coding algorithm to perform faster. But with smaller sliding window, the patches might miss some of the salient information. After trial and error, appropriate sliding window size of $8 \times 8$ is chosen so that the important features of the low dose images are not being missed. And the patches are chosen without any overlapping. We set the stopping criterion as global representation error $\varepsilon=0.01$. Threshold value used in the proposed method is 0.6 and 16 atoms are used to represent the images sparsely.

Performance of the proposed algorithm is compared with well known methods like OMP fusion and SOMP fusion methods. SCST-OMP is the extended version of SOMP fusion method with the following differences: 1) Fusion algorithm in [52] is developed using Overcomplete DCT dictionary of size $64 \times 256$ which is shown in Figure 6.6.(a). 2) Core idea of SOMP is to extract one atom in each stage.

Proposed algorithms are developed in MATLAB GUI 2013 environment for measuring the realistic computational time. Experiment is performed on natural images and some of the provided CT phantoms with the assumption that they all have common representation format. 


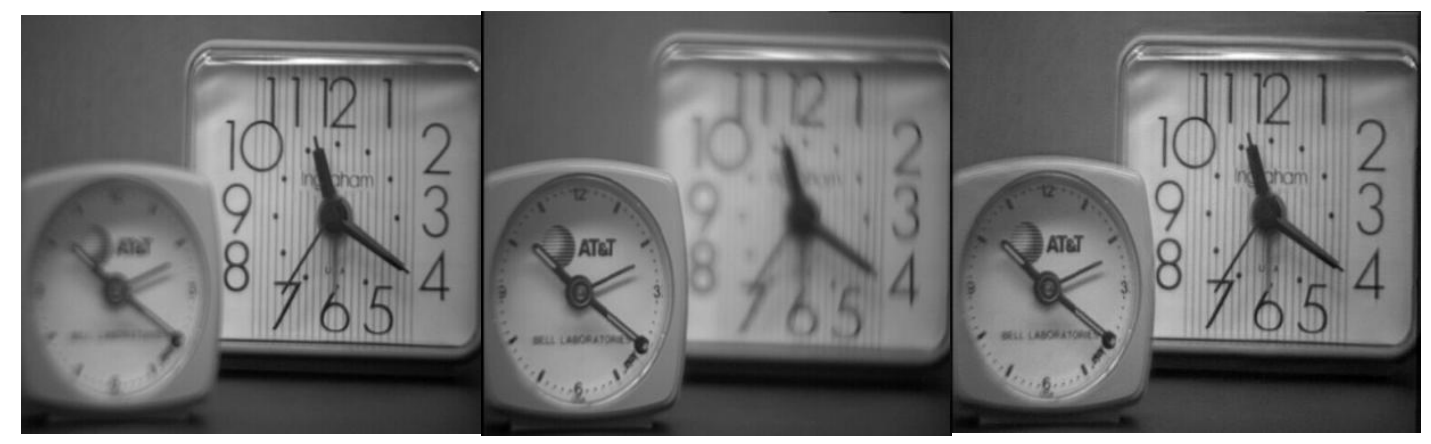

(1)

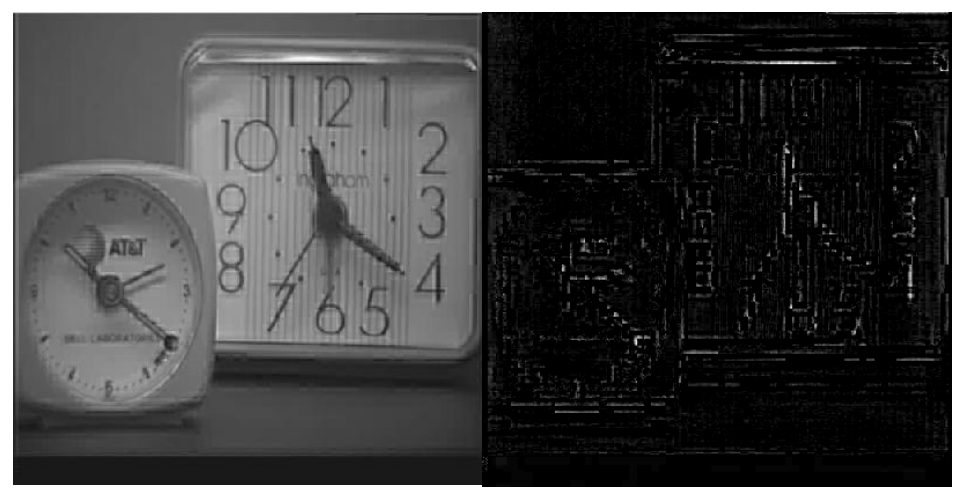

(2)

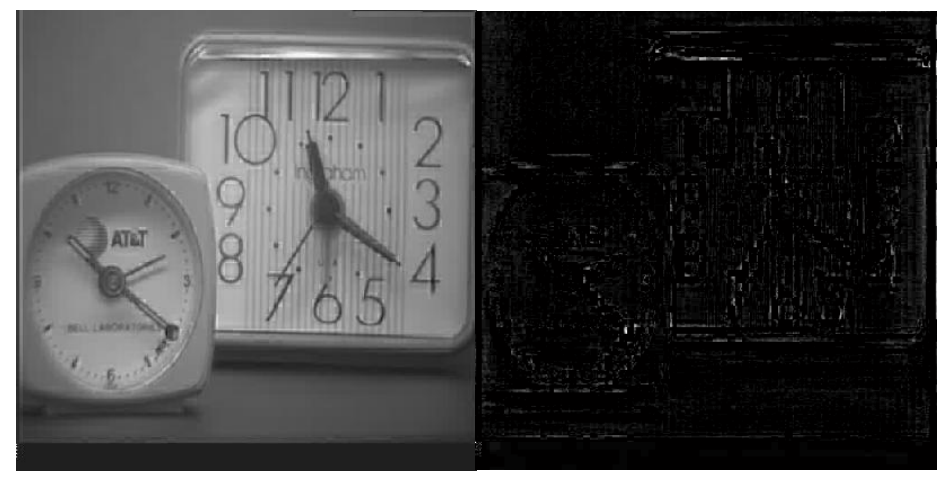

(3)

Figure 6.2. Displaying the fusion result and difference from reference of standard images using 1. Multifocus source images and a Reference image 2. OMP-DCT, 3.OMP-MDCT. 


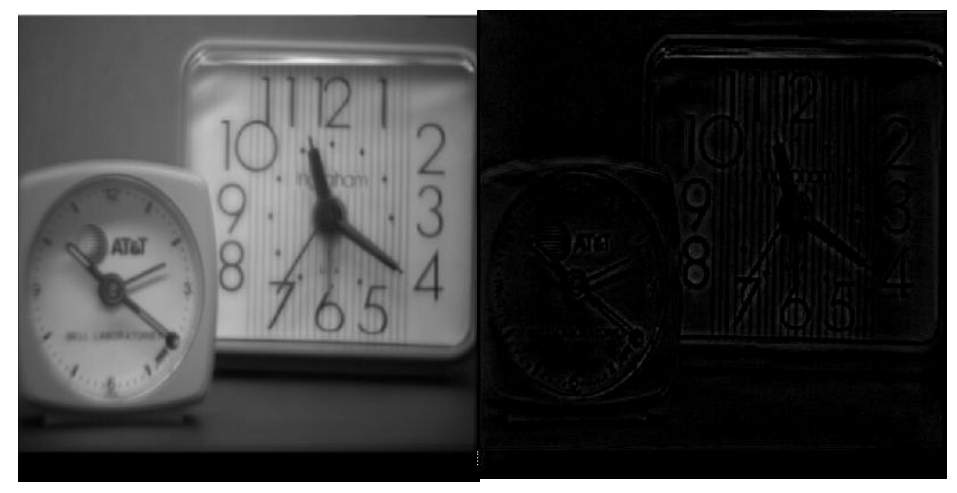

(4)

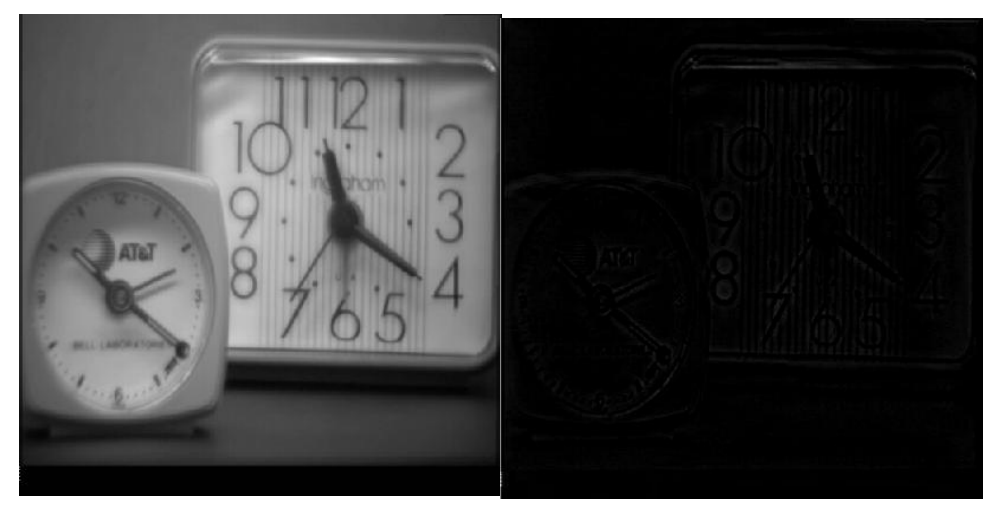

(5)

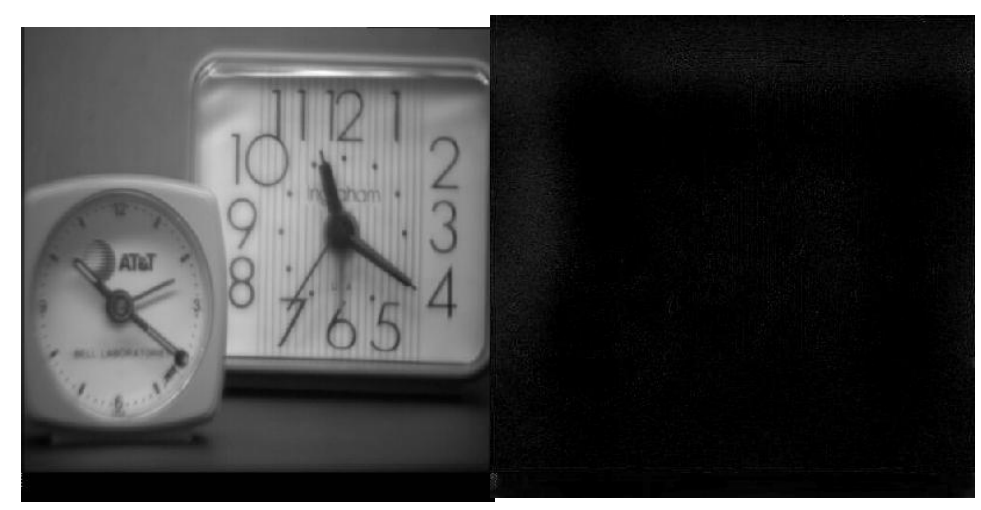

(6)

Figure 6.2. Displaying the fusion result and difference from reference of standard images using 4. SOMP-MDCT, 5. SCStOMP-DCT and 6. SCStOMP- MDCT. 
In order to validate the performance, we investigate the performance of the proposed method by performing the experiment on standard images and CT images. All the source images used were geometrically registered. Firstly the proposed method is experimented on multi focus clock images. First row of the Figure 6.1 presents the source images having two clocks to be fused and a reference image. First source image is captured by focusing the big clock and second source image is captured by focusing the small clock. Visually, the fusion result of OMP-DCT and OMP-MDCT seem to have low spatial resolution and the difference is more obvious. We can see that OMP-DCT fusion result contains artifacts. Results using simultaneous fusion methodology have clear details and better spatial resolution than OMP fusion methods. Proposed fusion method utilized MDCT dictionary contain clear clocks.

Table 6.1. Performance evaluation of proposed method for standard images quantitatively

\begin{tabular}{|c|c|c|c|c|c|c|}
\hline Source Images & Methodology & $\begin{array}{l}\text { PSNR } \\
(\mathrm{db})\end{array}$ & $\bar{H}$ & SSIM & Correlation & $\overline{M I}$ \\
\hline \multirow{5}{*}{$\begin{array}{c}\text { Clock } \\
\text { (Multifocus) }\end{array}$} & OMP-DCT & 28.8290 & 3.2442 & $\overline{0.8966}$ & 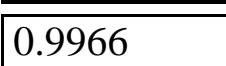 & \begin{tabular}{|l|}
0.5011 \\
\end{tabular} \\
\hline & $\overline{\text { OMP-MDCT }}$ & 29.9021 & 3.1751 & 0.8999 & 0.9973 & 0.5147 \\
\hline & $\overline{\text { SOMP-MDCT }}$ & 31.2682 & 2.9144 & $\overline{0.9666}$ & 0.9980 & $\overline{\mid 0.5142}$ \\
\hline & $\overline{\text { SCSt-OMP DCT }}$ & 31.5374 & 2.9239 & 0.9667 & 0.9981 & 0.5164 \\
\hline & SCSt-OMP MDCT & $\overline{\mathbf{3 1 . 5 4 2 0}}$ & 2.9252 & 1.9669 & 0.9981 & \begin{tabular}{|l|}
0.5205 \\
\end{tabular} \\
\hline
\end{tabular}

The objective evaluation on the fusion results of all the methods with reference image of the clock image is listed in Table 6.1. Best quantitative results are represented in bold. Proposed method outperforms other methods quantitatively. Simultaneous fusion methods perform way better than the OMP fusion methods qualitatively and quantitatively. 


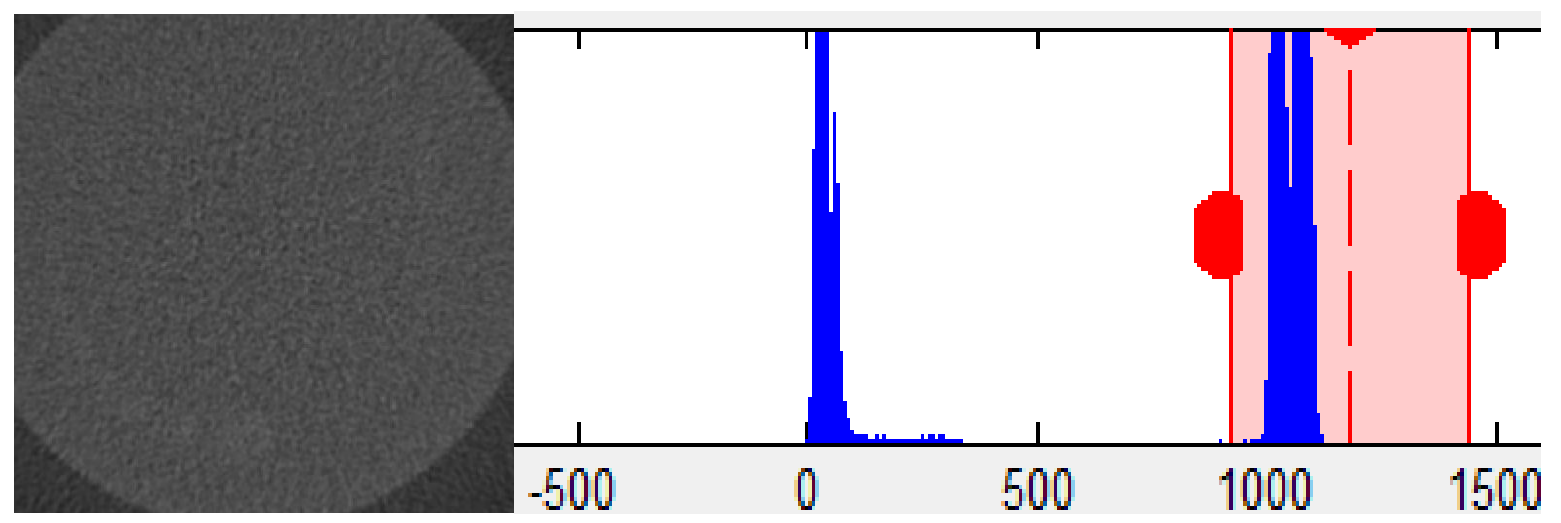

(1)

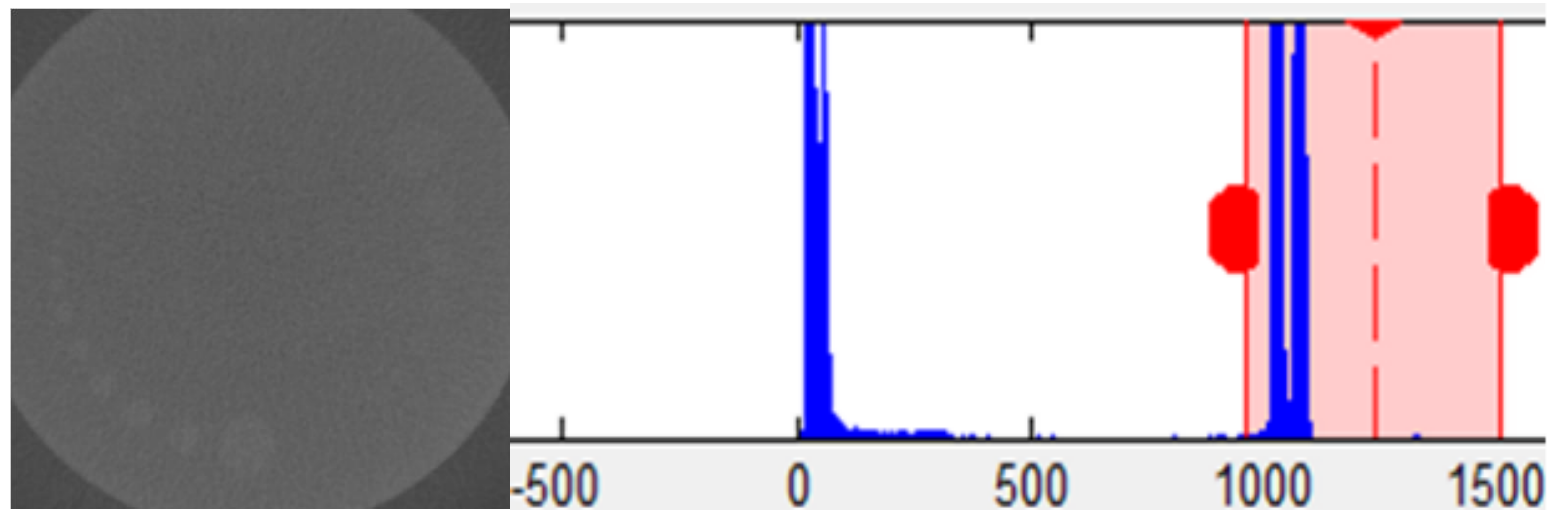

(2)

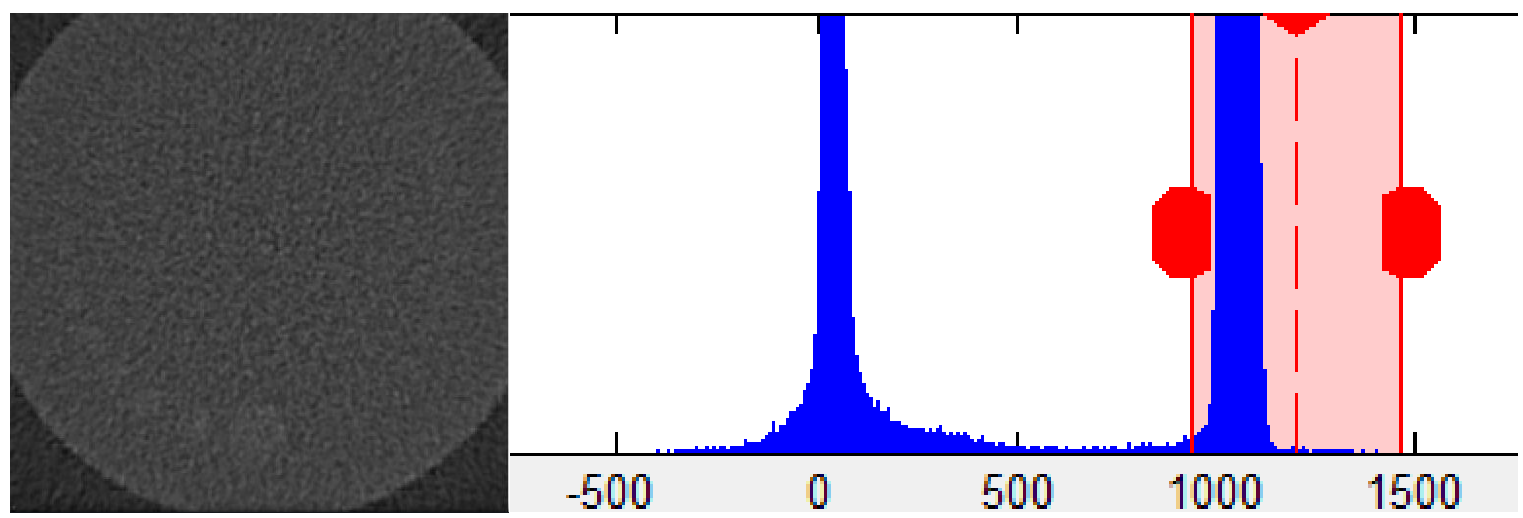

(3)

Figure 6.3. Displaying the fusion result and contrast map using 1. Low dose image, 2.Highdose image as reference, 3. OMP-DCT. 


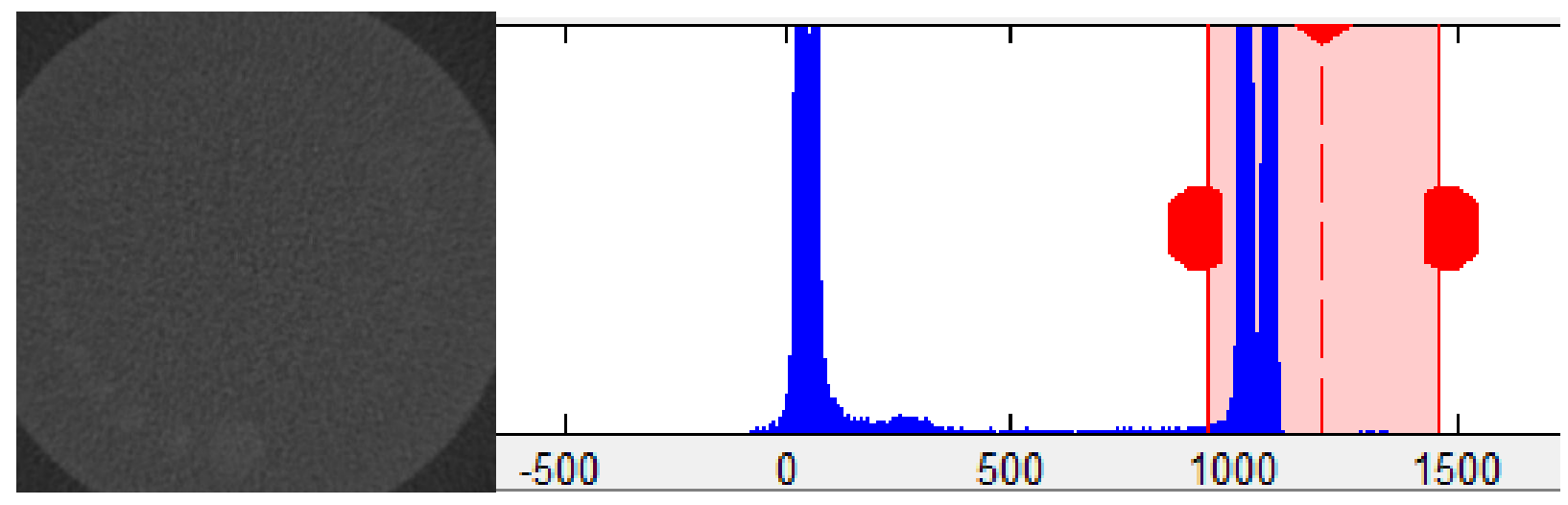

(4)

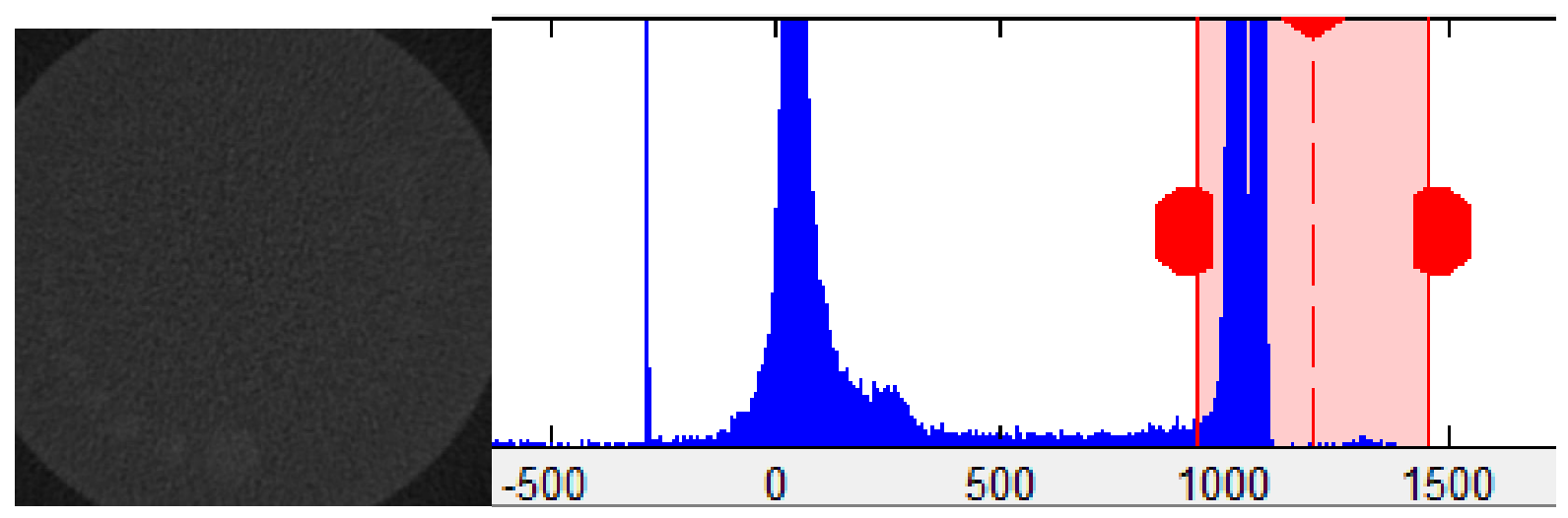

(5)

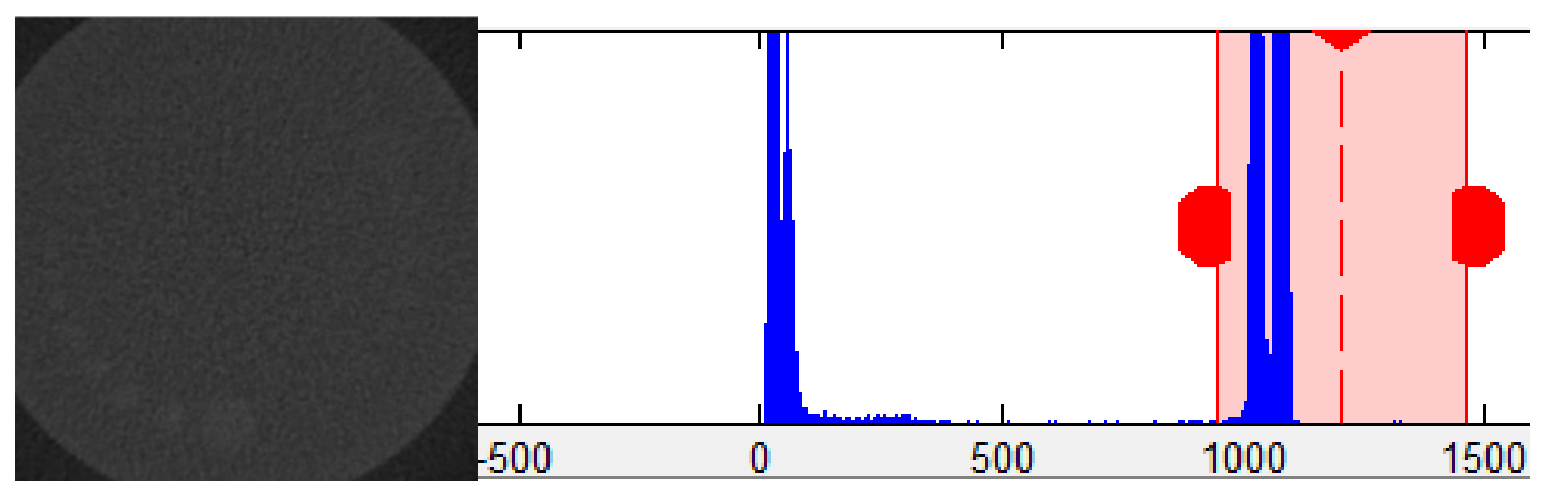

(6)

Figure 6.3. Displaying the fusion result and contrast map using 4.OMP-MDCT, 5. SOMPMDCT, 6. SCSt-OMP utilizing Overcomplete DCT. 


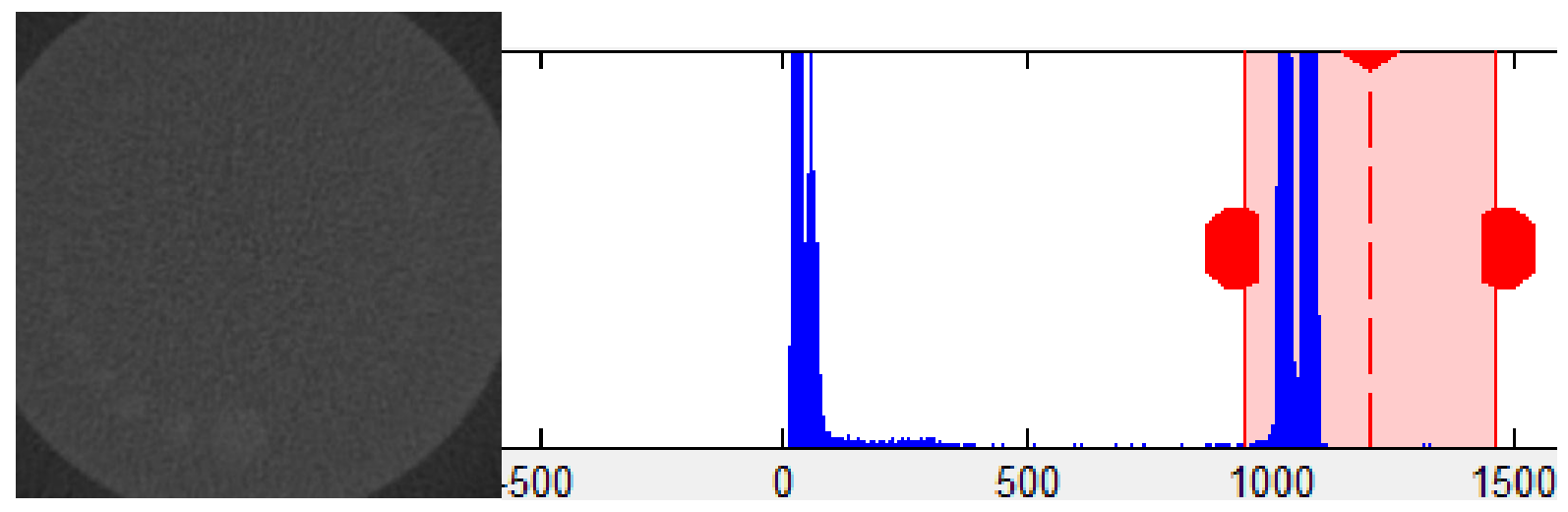

(7)

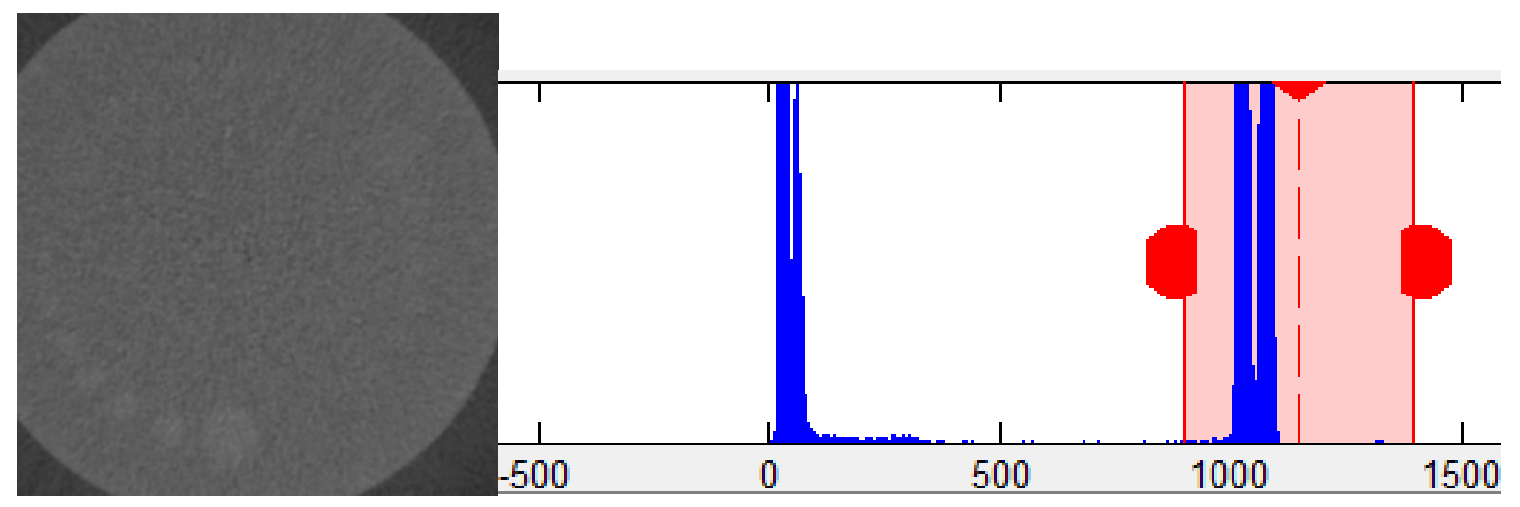

(8)

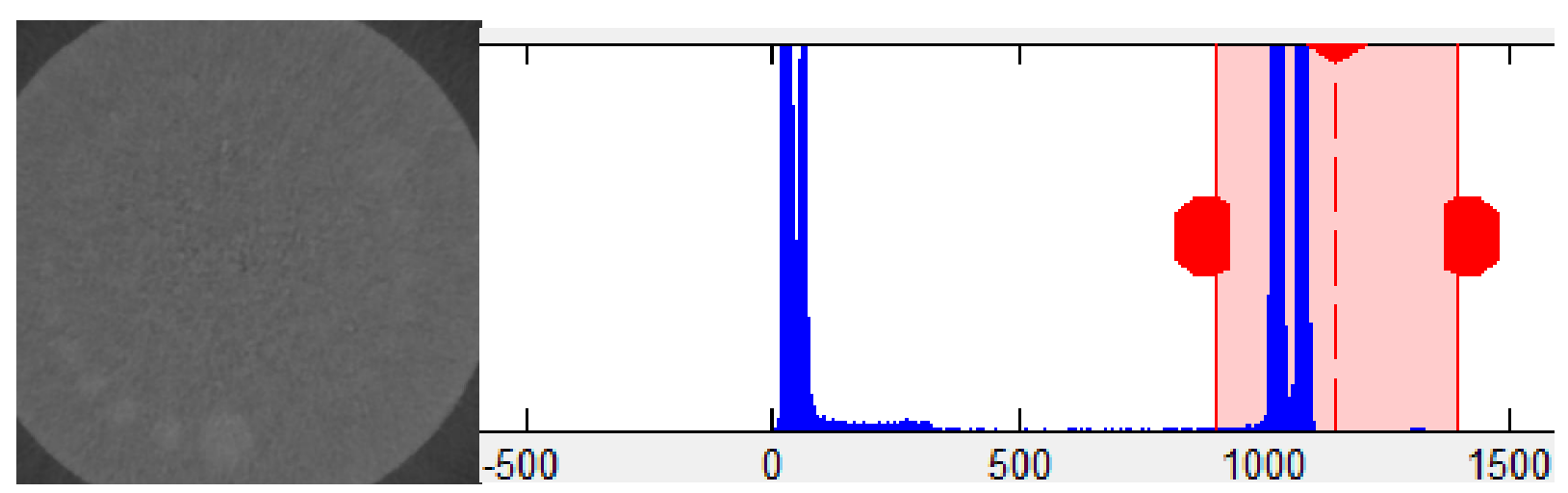

(9)

Figure 6.3. Displaying the fusion result and contrast map using 7. SCSt-OMP utilizing MDCT (Contribution I), 8. Joint Sparse PCA model (Contribution II), 9. Focussed Region fusion (Contribution III) 
Table 6.2. Performance evaluation of proposed method for CT images quantitatively

\begin{tabular}{|c|c|c|c|c|c|c|}
\hline Source Images & Methodology & $\begin{array}{l}\text { PSNR } \\
(\mathrm{db})\end{array}$ & $\bar{H}$ & SSIM & Correlation & MI \\
\hline \multirow{7}{*}{$\begin{array}{c}\text { Low dose } \\
\text { and } \\
\text { medium dose } \\
\text { Phantoms }\end{array}$} & OMP-DCT & 28.8290 & 3.2442 & 0.8966 & 0.9966 & 0.5011 \\
\hline & OMP-MDCT & 29.9021 & 3.1751 & 0.8899 & 0.9973 & 0.5147 \\
\hline & SOMP-MDCT & 31.2682 & 2.9144 & 0.8899 & 0.9980 & 0.5142 \\
\hline & $\begin{array}{l}\text { SCSt-OMP DCT } \\
\end{array}$ & 31.5374 & 2.9239 & 0.8999 & 0.9981 & 0.5164 \\
\hline & SCSt-OMP MDCT & 32.7969 & 0.6780 & 0.8947 & 1.0000 & 0.5174 \\
\hline & Sparse PCA Joint Model & 34.7480 & 0.7781 & 0.9800 & 1.0000 & 0.7764 \\
\hline & Region focused Fusion & 34.9423 & 0.7780 & 1.9880 & 1.0000 & 0.7861 \\
\hline
\end{tabular}

Figure 6.3 shows the results of integrating low dose and medium dose CT phantom images using different methodologies. Low dose image in (1) of Figure.6.3 seems to be very noisy and the noise hides some details especially the circles. Our proposed algorithm is implemented to fuse $60 \%$ dose image (low dose) and 90\% dose (medium dose) image. Results of fusion using various methods are shown in Figure 6.3.Visually, fusion result of the Contribution I is better than the existing algorithms since the circle details on the left side are discernible. Contrast plot of Contribution I and sparse method is similar to the contrast map of high dose image as compared to other conventional fusion methods. SOMP fusion results are also better compared to conventional OMP method but contribution I shows superior results than SOMP fusion. 
These experiments on these low dose and medium dose CT phantom images illustrates that the proposed method outperforms other existing fusion algorithms quantitatively as shown in Table 6.2.

Use of "max-abs rule" in all OMP, SOMP fusion methods has considered the enhanced details from both the images but noise is not removed. So these methods discussed in this section can give promising results only on clean images. A good threshold in each iteration stage and MDCT dictionary results in good sparse reconstruction of proposed SCSt-OMP and ensures better fusion results compared to already existing fusion methods.
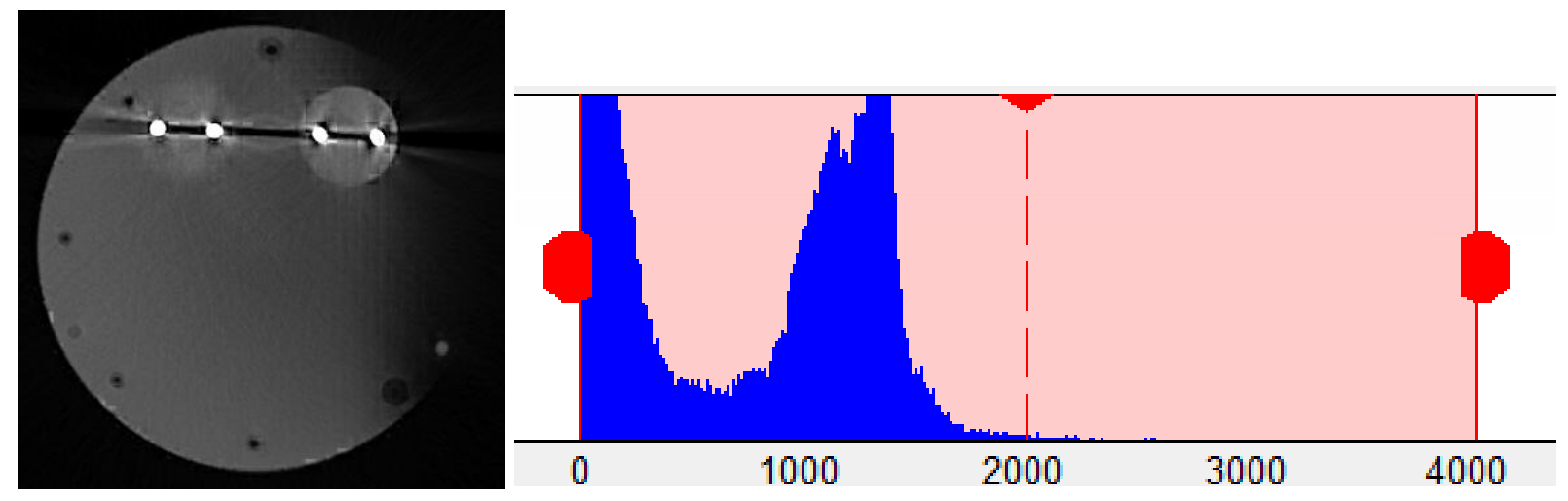

(1)
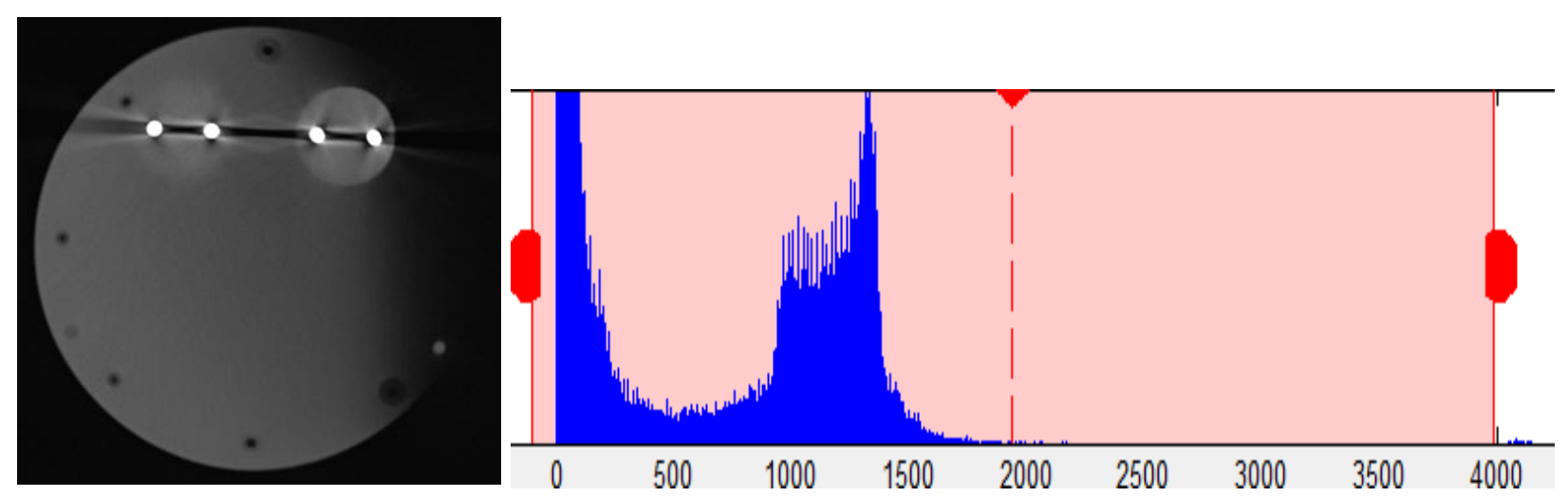

(2)

Figure 6.4. Displaying the fusion result and contrast map using 1. Low dose image, 2.Highdose image as reference. 

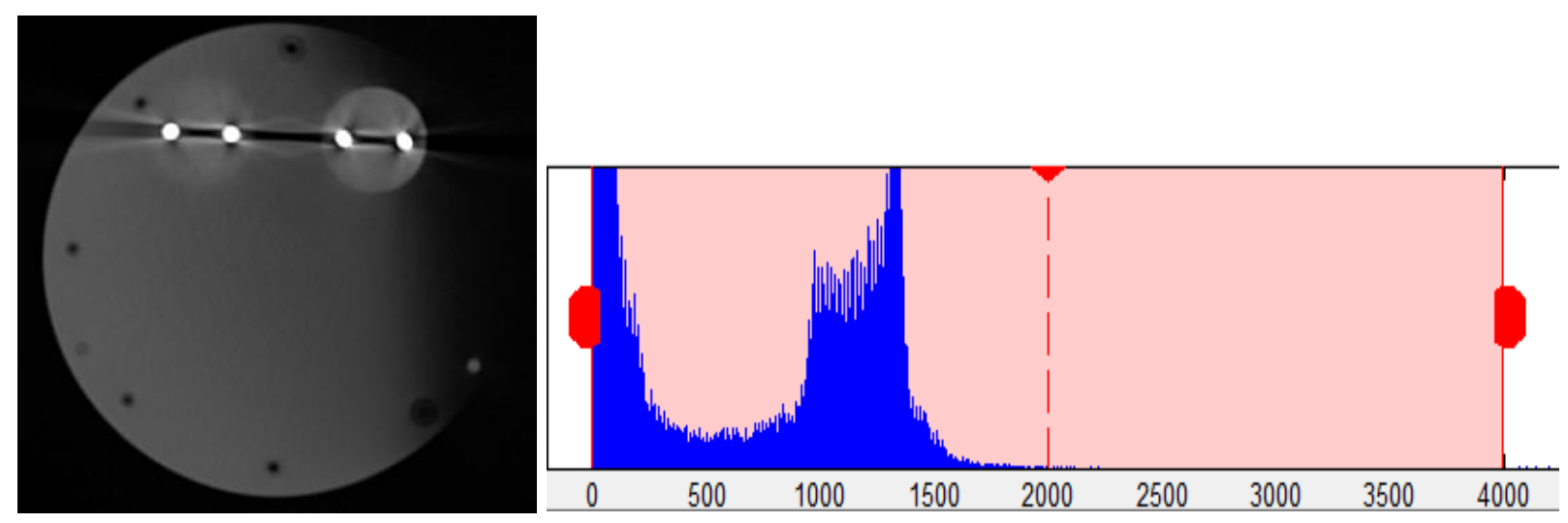

(3)
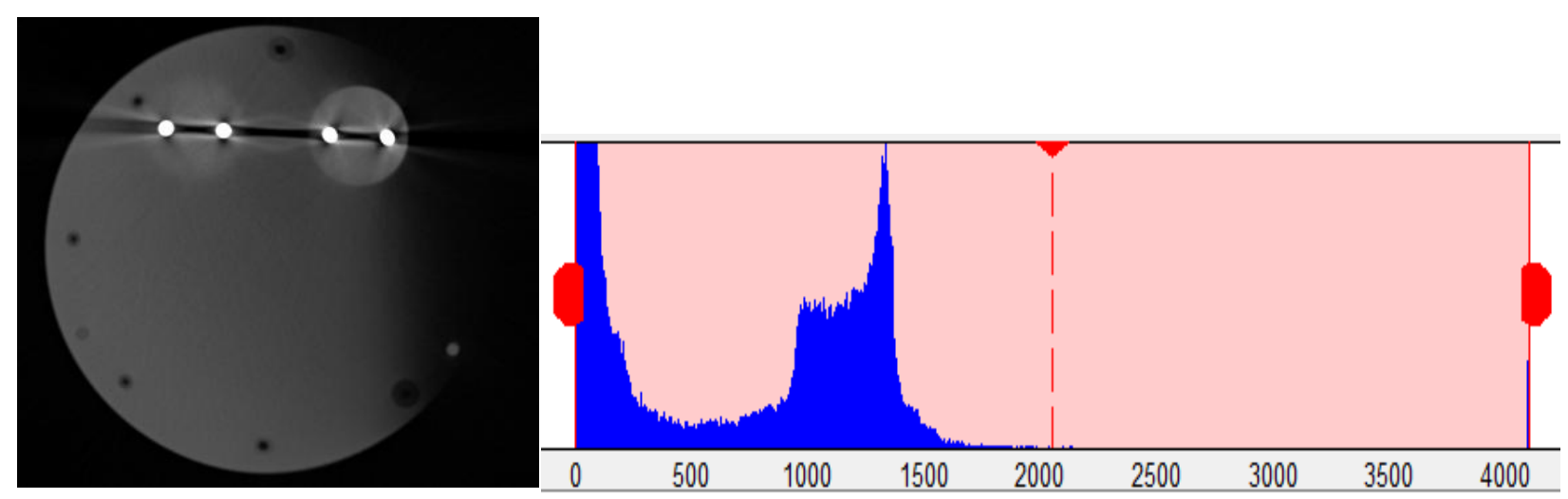

(4)
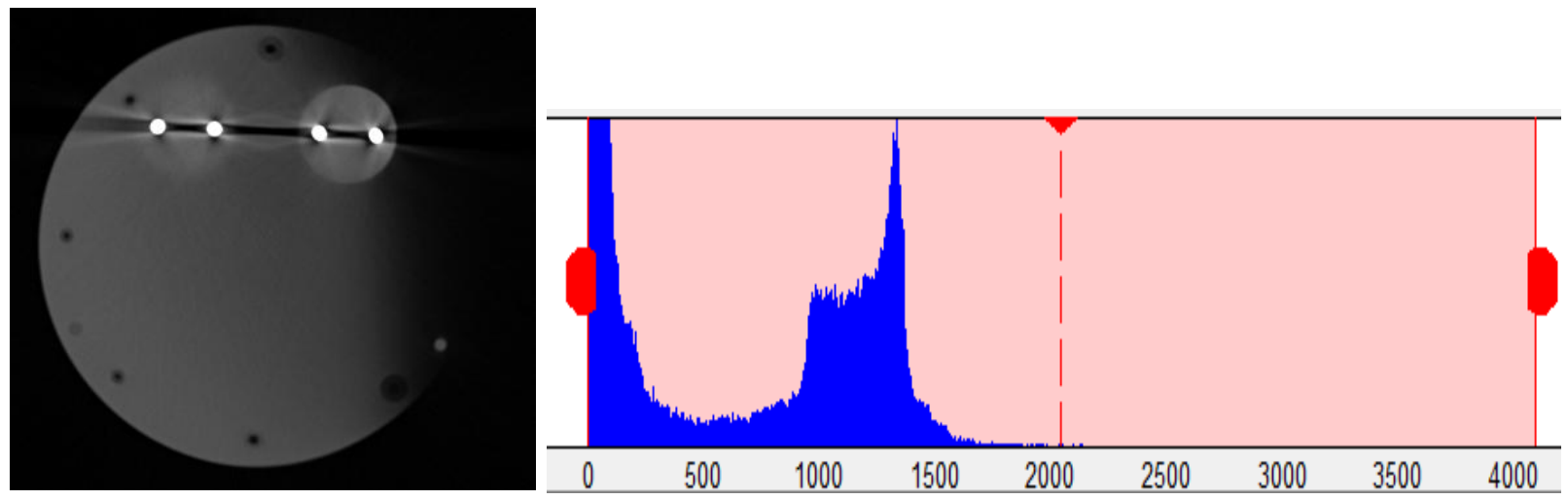

(5)

Figure 6.4. Displaying the fusion result and contrast map using 3.OMP-MDCT, 4. SCSt-OMP utilizing Overcomplete DCT and 5. SCSt-OMP utilizing MDCT. 
Table 6.3. Performance evaluation of proposed method for CT images quantitatively

\begin{tabular}{|c|c|c|c|c|c|c|}
\hline Source Images & PSNR (db) & $\bar{H}$ & MI & Correlation & SSIM & CNR \\
\hline \multirow{6}{*}{$\begin{array}{l}\text { Low dose and } \\
\text { Medium dose } \\
\text { Phantoms }\end{array}$} & OMP-DCT & 16.4079 & 0.1982 & 0.2494 & 0.9840 & 0.174 \\
\hline & OMP-MDCT & 21.4514 & 0.1260 & 0.3762 & 0.9846 & 0.1831 \\
\hline & & & & & & \\
\hline & SOMP MDCT & 30.2658 & 0.1274 & 0.3924 & 0.9844 & 0.2264 \\
\hline & SCSt-OMP DCT & 31.3789 & 0.1131 & 0.8738 & 0.9888 & 0.3338 \\
\hline & \begin{tabular}{|l} 
SCSt-OMP MDCT \\
\end{tabular} & $\mathbf{3 4 . 3 7 3 2}$ & 0.1011 & 1.0000 & 0.9889 & 0.5174 \\
\hline
\end{tabular}

Figure 6.4 shows the results of integrating low dose and medium dose CT phantom images using different methodologies. Our proposed algorithm is implemented to fuse $60 \%$ dose image (low dose) and 90\% dose (medium dose) image. Results of fusion using various methods are shown in Figure 6.3. Visually, fusion result of proposed method is similar to high dose image. Contrast plot of proposed method and sparse method is similar to the contrast map of high dose image. It is observed that the result of conventional OMP fusion methods shows artifacts and the results are disappointing visually. Fused result seems to contain the details available in low dose and medium dose images without the introduction of artifacts. From Table 6.3, Quantitative results of all the other methods except proposed seems to be really low for the existing methods. Choosing the threshold as 0.6 makes this algorithm computationally efficient. 


\subsection{Experimental setup and Results of Contribution II and Contribution III}

Under this section, the experiments are performed on low dose and medium dose CT images using proposed Joint Sparse PCA and fusion using focused vector. As similar to previous section, the same five evaluation criteria are used to assess the quality of the fused image. Learning-based dictionaries are used for efficient sparse representation than fixed dictionaries. We use a patch size of $8 \times 8$ and the dictionary learned from high dose images has the size $64 \times 256$. For the noise intensity of $\sigma$, we set the stopping error criterion as $1.15 \times \sqrt{128 \sigma}$.
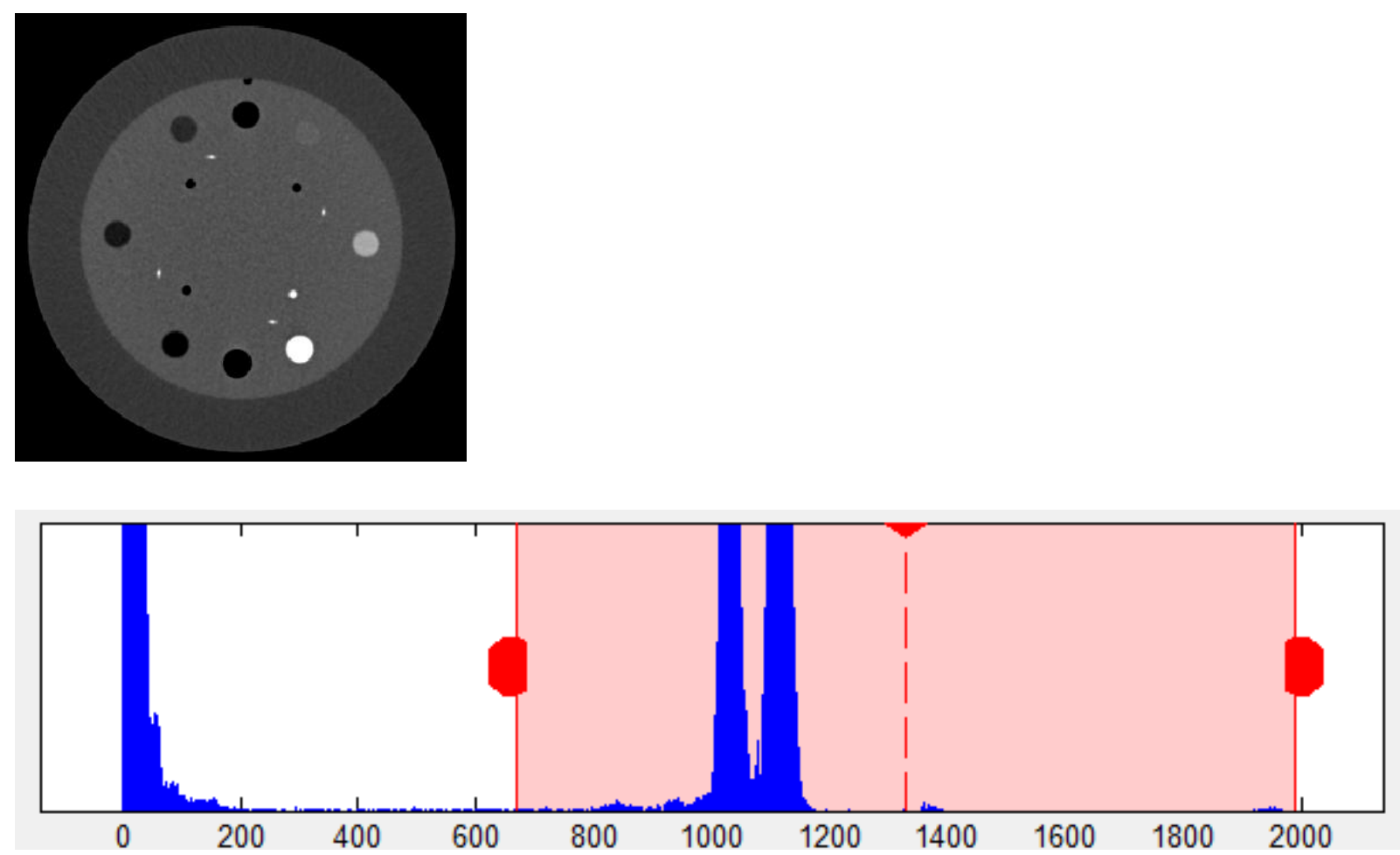

(1)

Figure 6.5. Displaying the fusion result and contrast map using 1. Low dose image (source image) 

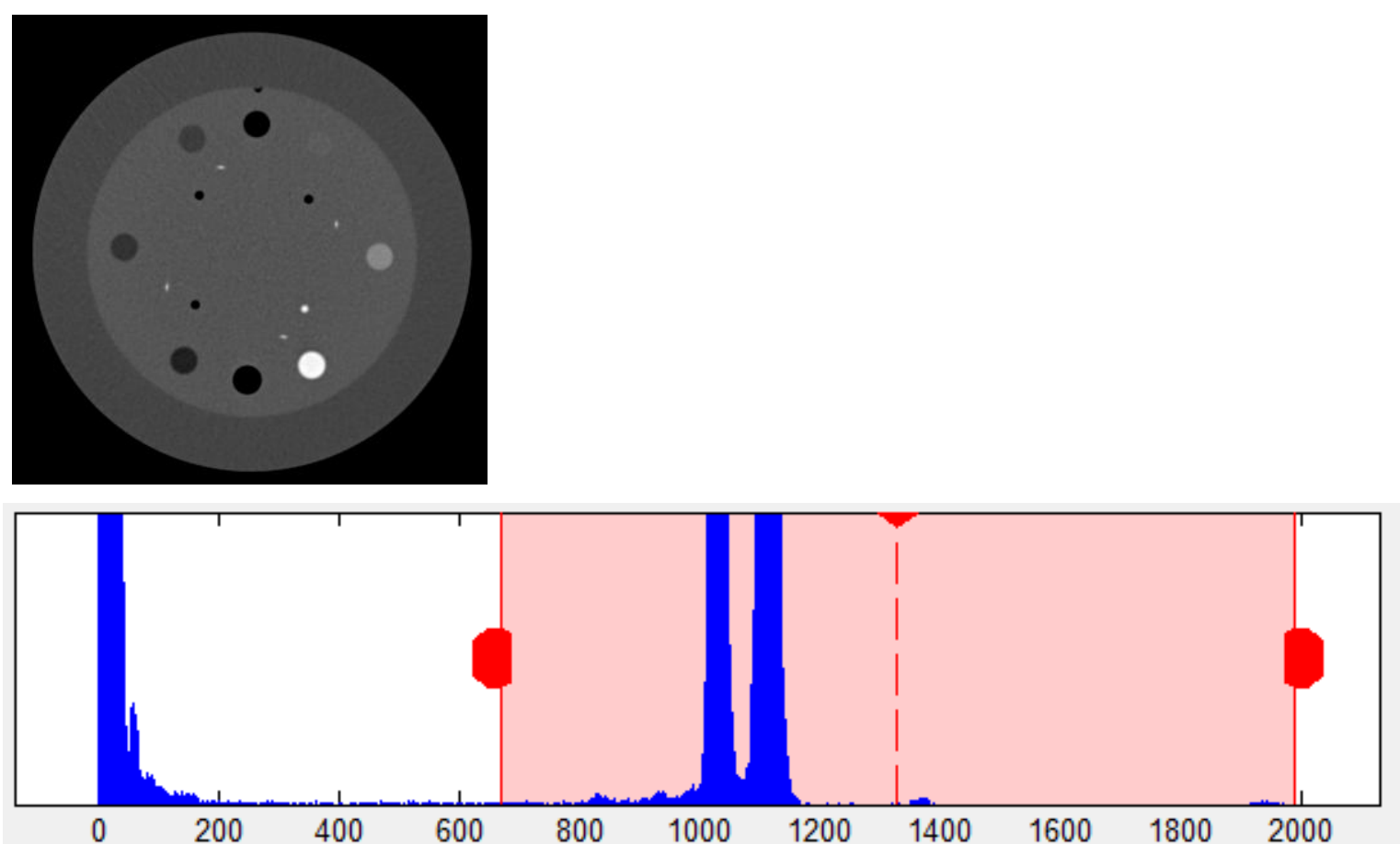

(2)
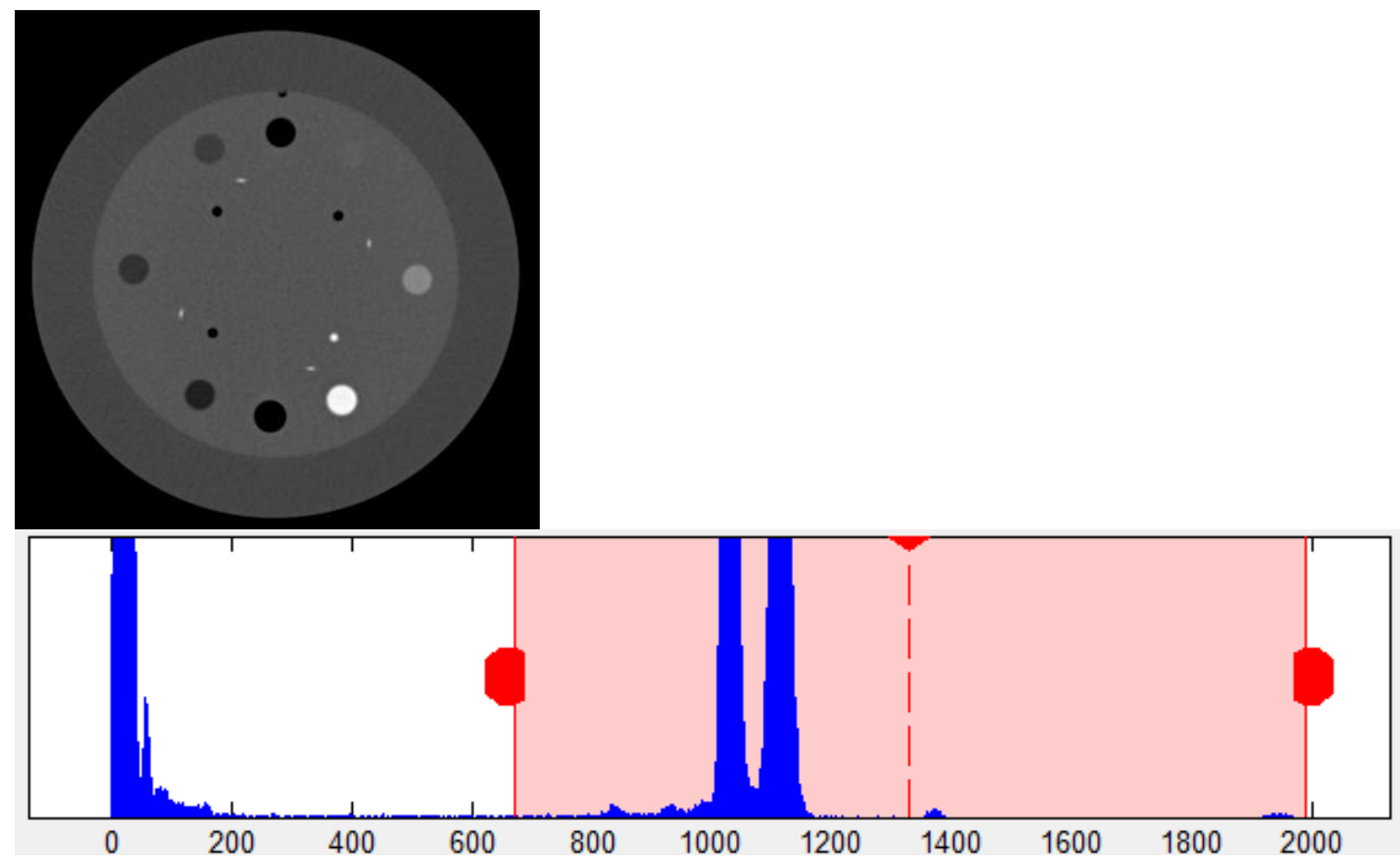

(3)

Figure 6.5. Displaying the fusion result and contrast map using 1. Low dose image (source image), 2.Medium dose image (source image), 3. High dose image (reference image). 

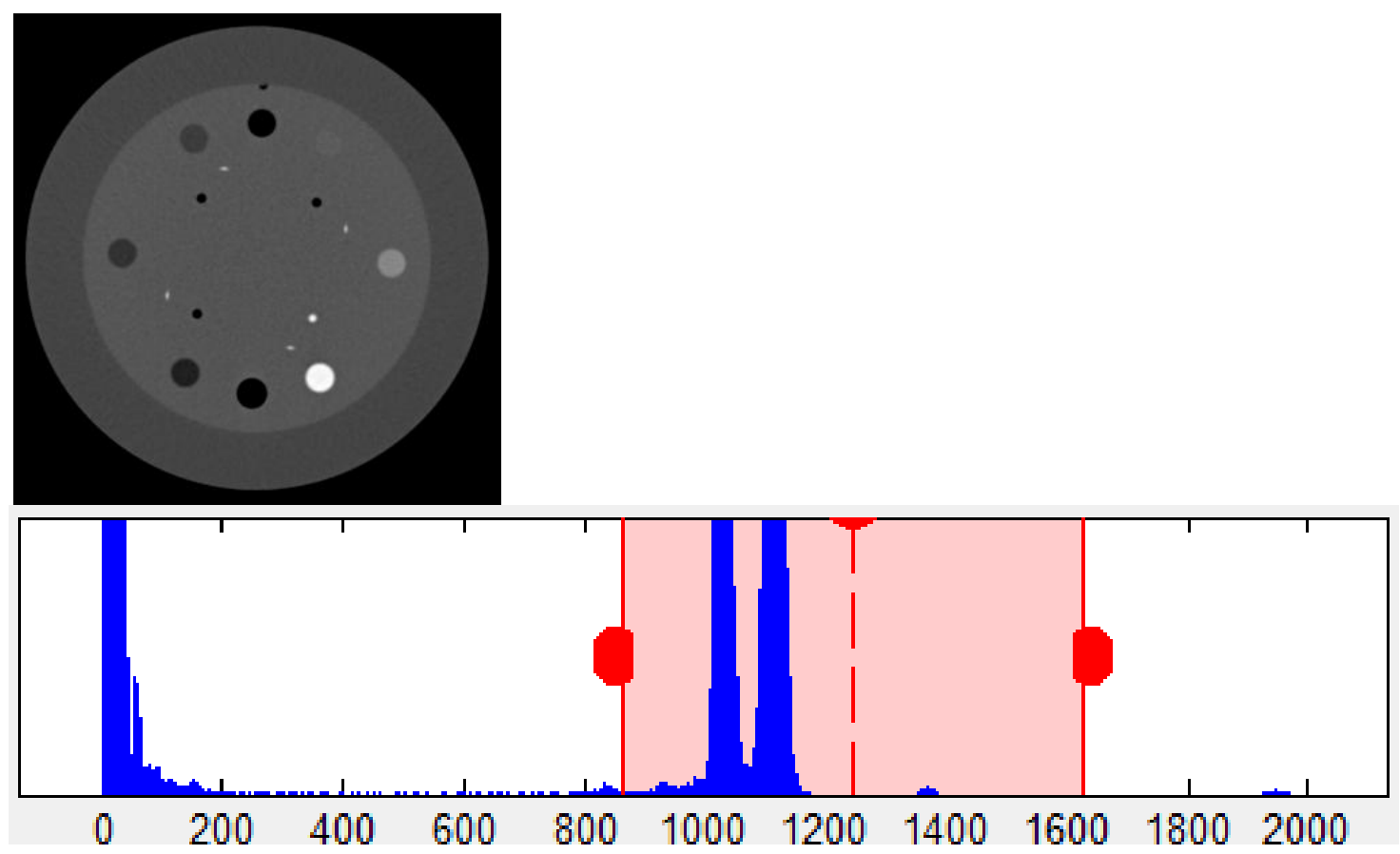

(4)
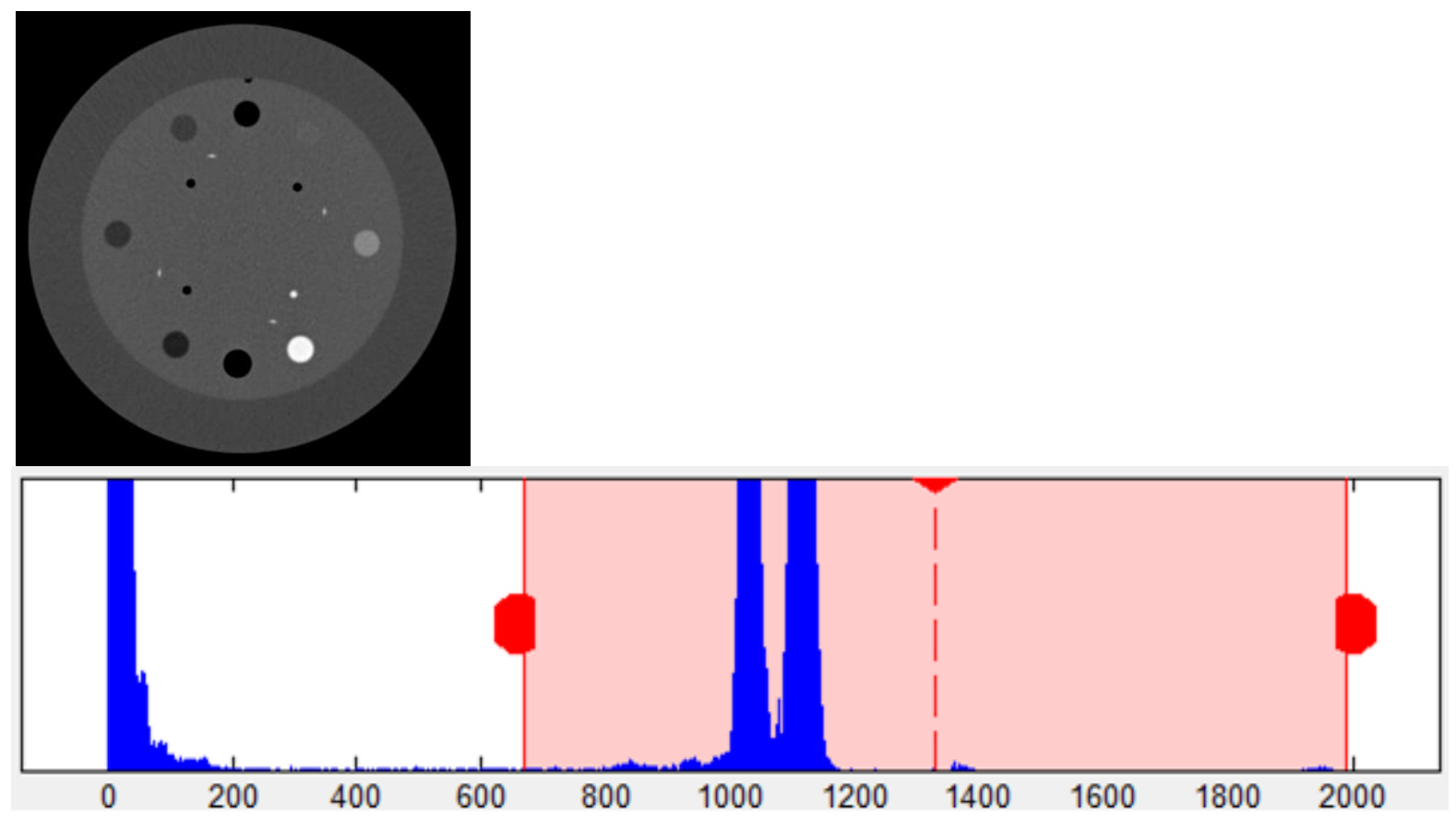

(5)

Figure 6.5. Displaying the fusion result and contrast map using 4.Focussed Region Fusion, 5. CSt-OMP fusion 

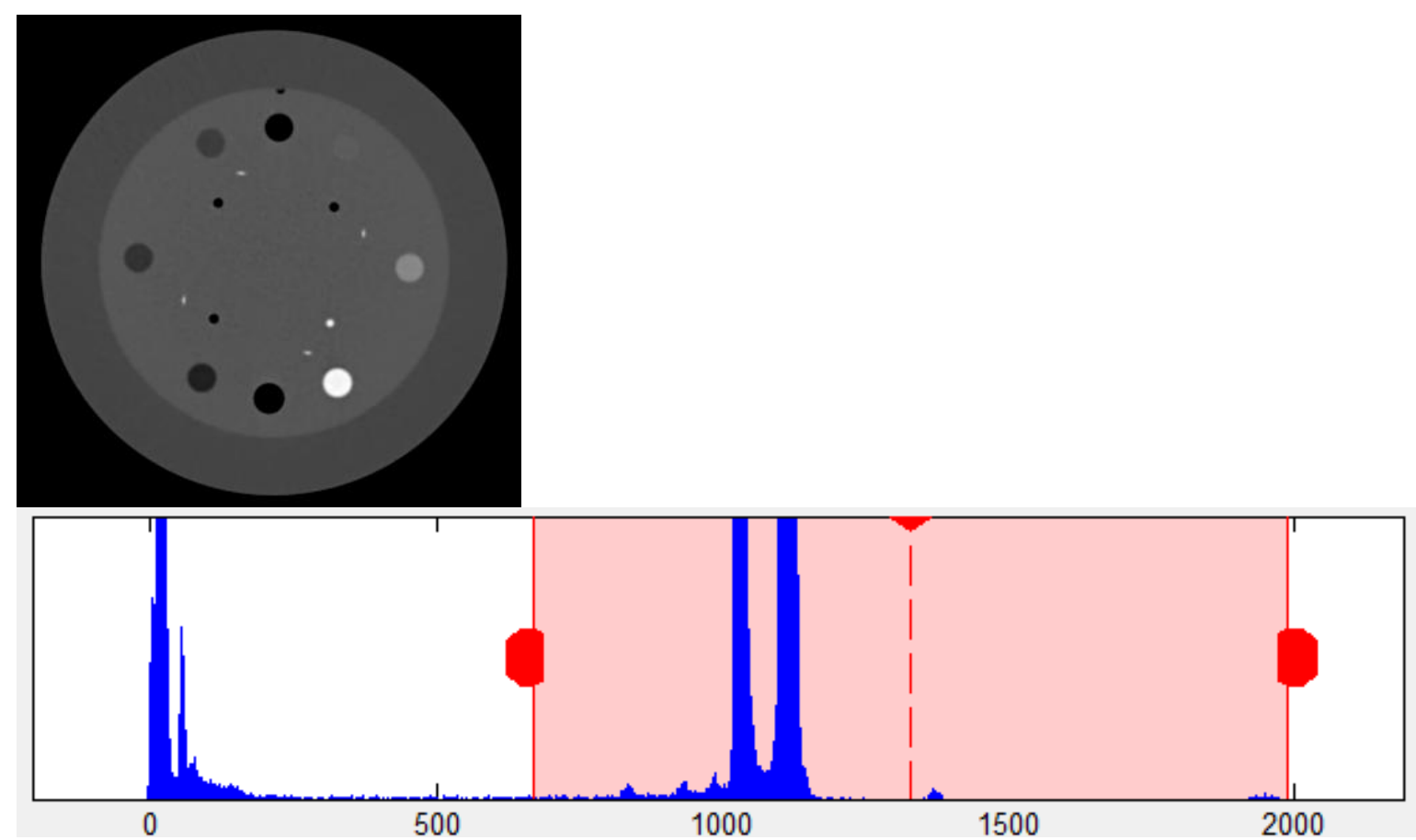

(6)

Figure 6.5. Displaying the fusion result and contrast map using 6. Joint Sparse PCA fusion

Table 6.4. Performance evaluation of proposed method for CT images quantitatively

\begin{tabular}{|c|c|c|c|c|c|c|}
\hline Source Images & PSNR (db) & $\bar{H}$ & MI & Correlation & SSIM & CNR \\
\hline \multirow{3}{*}{$\begin{array}{l}\text { Low dose and } \\
\text { Medium dose } \\
\text { Phantoms }\end{array}$} & CSt-OMP fusion & 27.7356 & 0.0357 & 0.999 & 0.9609 & 23.4655 \\
\hline & $\begin{array}{l}\text { Joint Sparse PCA } \\
\text { fusion }\end{array}$ & 27.7925 & 0.0418 & 0.999 & 0.9644 & 22.5055 \\
\hline & $\begin{array}{ll}\text { Focused } & \text { Region } \\
\text { fusion } & \end{array}$ & 29.5398 & 0.035 & 1.0000 & 0.9777 & 20.5025 \\
\hline
\end{tabular}

Figure $6.3(6)$ and (7) are the fused images of Joint Sparse PCA fusion and Region focused image fusion methods. All the details are clearly discernible. Especially the circular 
rings on the left are visible and obviously better than the existing methods. Circular rings on right side are also slightly visible which are not at all visible in other fusion results. Contrast map of Contribution II and Contribution III are even better. Especially, the contrast map of contribution III is almost same as that of high dose contrast map.

Objective evaluations are presented in Table 6.4. It is observable that all the image quality metrics value show superior results for the contributed works. CNR is the important quantity metrics evaluation for medical images which is better for the contributed methods.

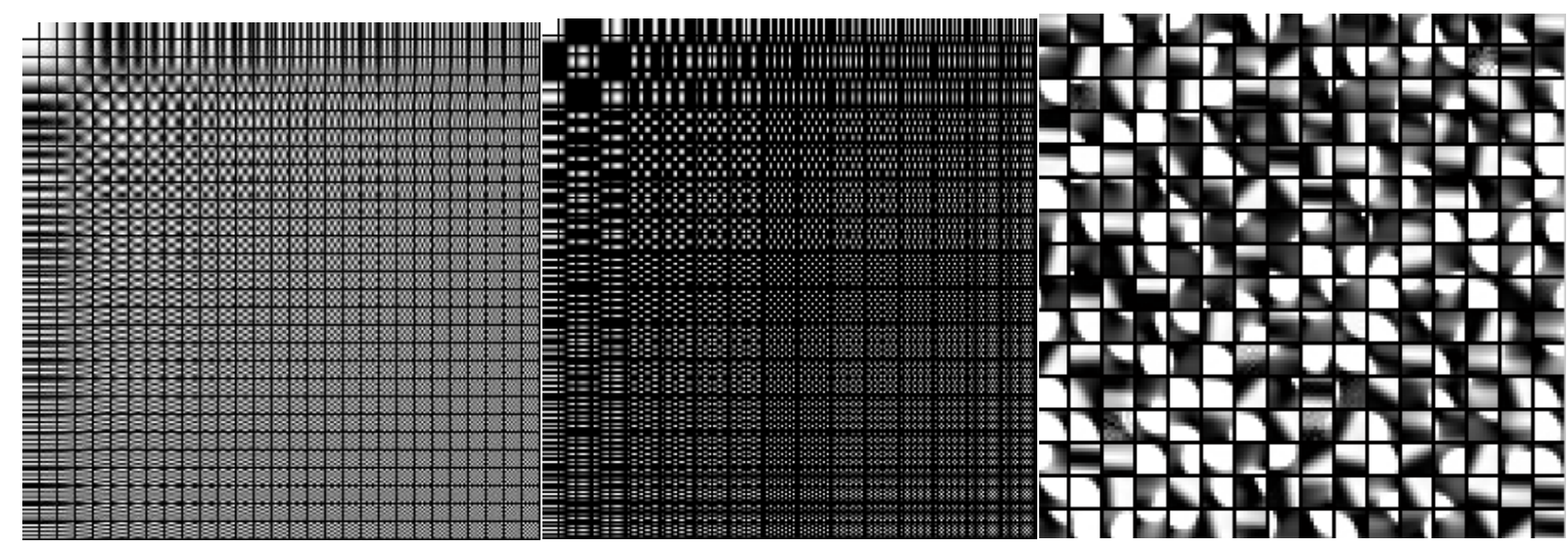

(a)

(b)

(c)

Figure 6.6. The overcomplete dictionaries: (a) Overcomplete DCT (b) MDCT Dictionary (c) Trained Overcomplete dictionary

Figure 6.5 depicts the results of integrating 60\% low dose and 90\% medium dose CT phantom images. Visually, Fusion result of Contributed methods, in particular, Contribution III is same as high dose image. Contrast plot of proposed all the three contributed methods are very similar to the contrast map of high dose image. Result of conventional OMP fusion methods shows noise and its contrast map is very similar to medium dose image.

Objective evaluation of Figure 6.5 results is presented in Table 6.3. From all the objective results presented, contributed methods are obviously better than other methods. Among the contributed methods, Contribution III gives out superior result since it denoises and fuses the information simultaneously. 


\subsection{Integration of unregistered images using Focused Region fusion}

To confirm the effective performance of the focused region based sparse fusion, further, unregistered low dose images of the same region of interest is fused. The fused result is evaluated using Contrast Noise Ratio (CNR) and contrast map. Maximum iteration of K-SVD is set to 40. High dose resolution CT set is incorporated during the training process for enhancing the details and the trained dictionary is shown in Figure 6.6.(c). Source images are two Cone Beam CTs (CBCT) of the same patient at two different times. The goal is to denoise the CBCTs using the high resolution CT as learning set.
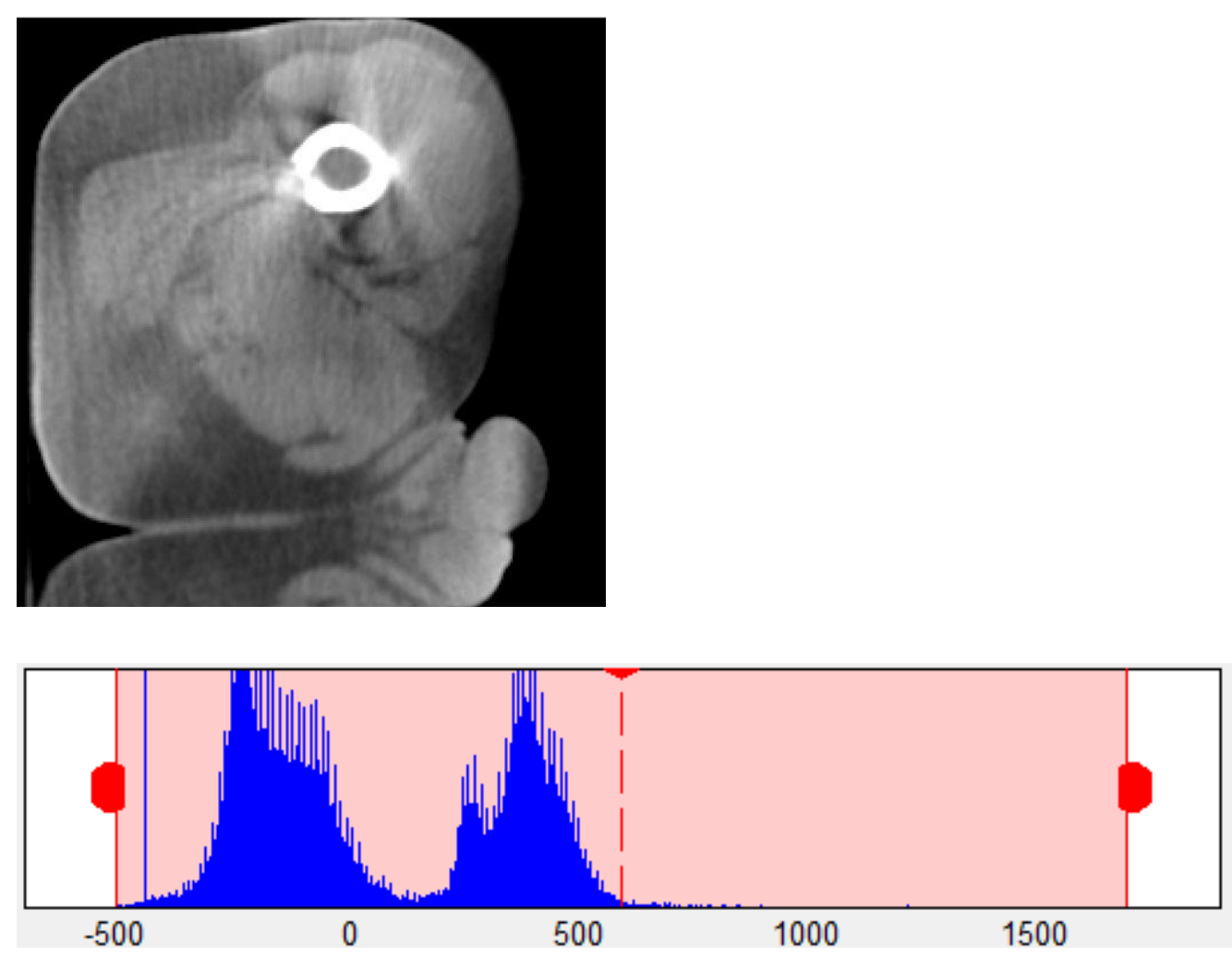

(1)

Figure 6.7. Displaying the fusion result and Contrast map of fusing two Cone Beam CTs (CBCT) 1. Low dose image with CNR 22.8330 


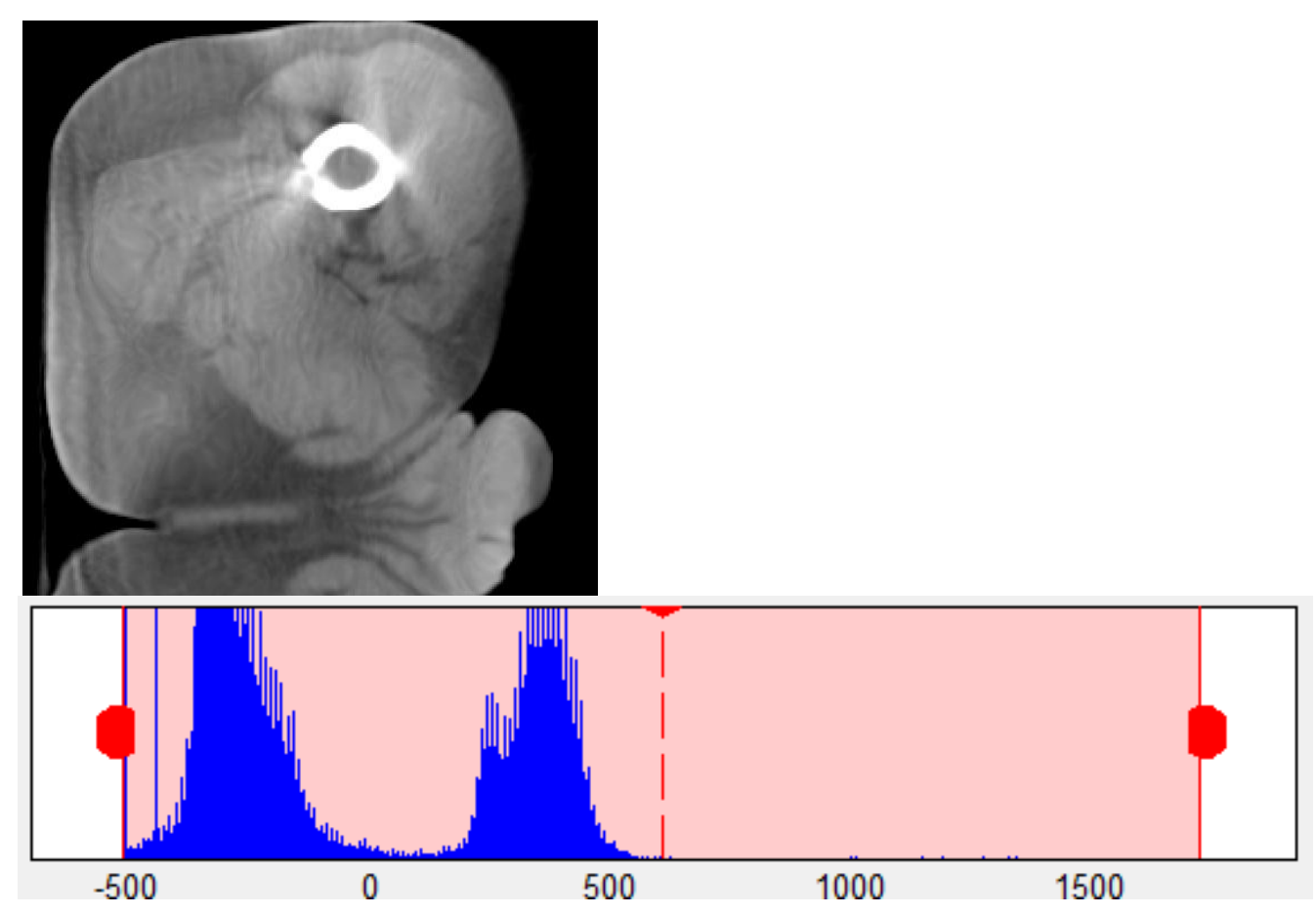

(2)
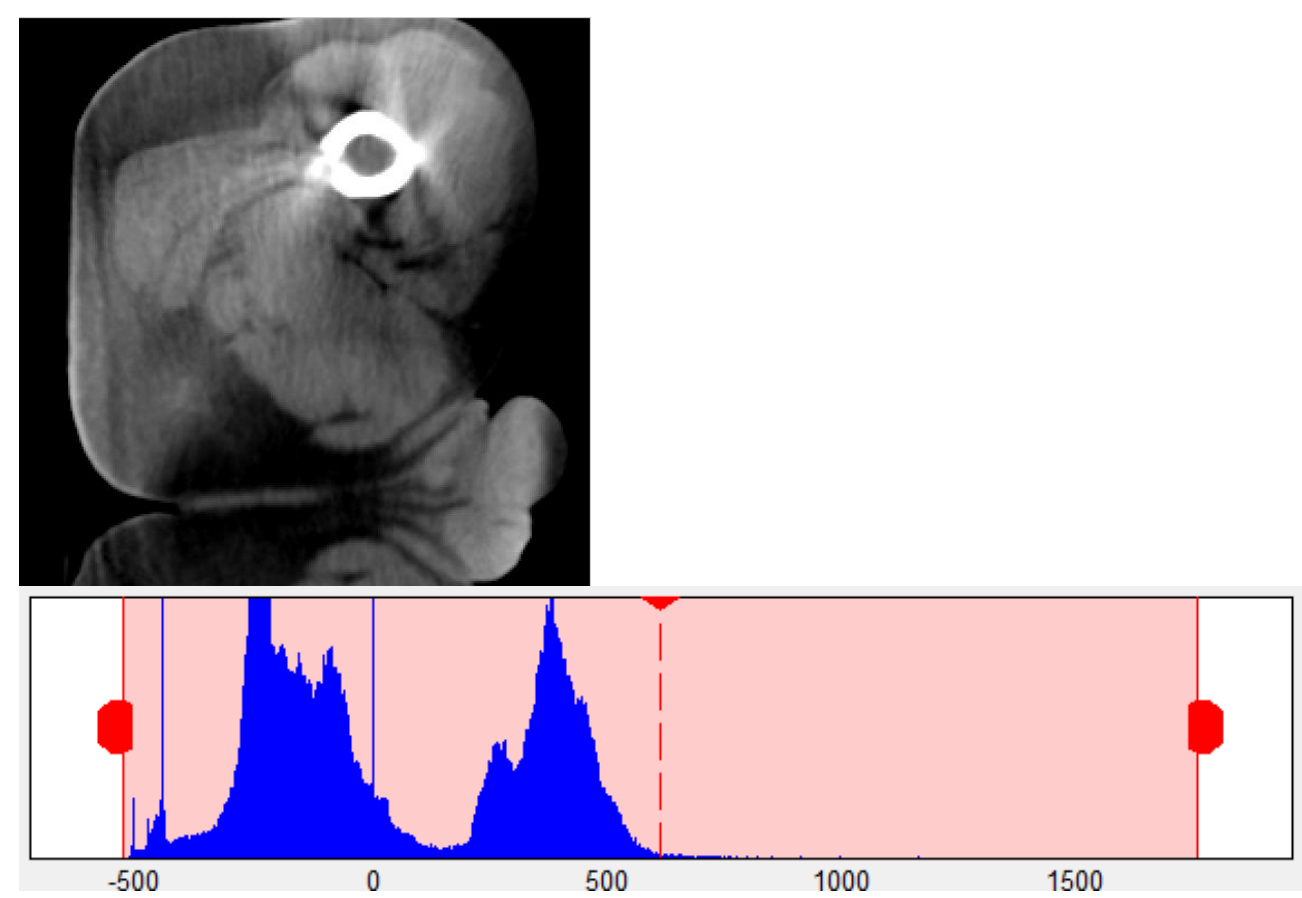

(3)

Figure 6.7. Displaying the fusion result and Contrast map of fusing two Cone Beam CTs (CBCT) 2. Another low dose image with CNR of 22.1172, 3.Fusion result of proposed method with CNR of 21.4806 

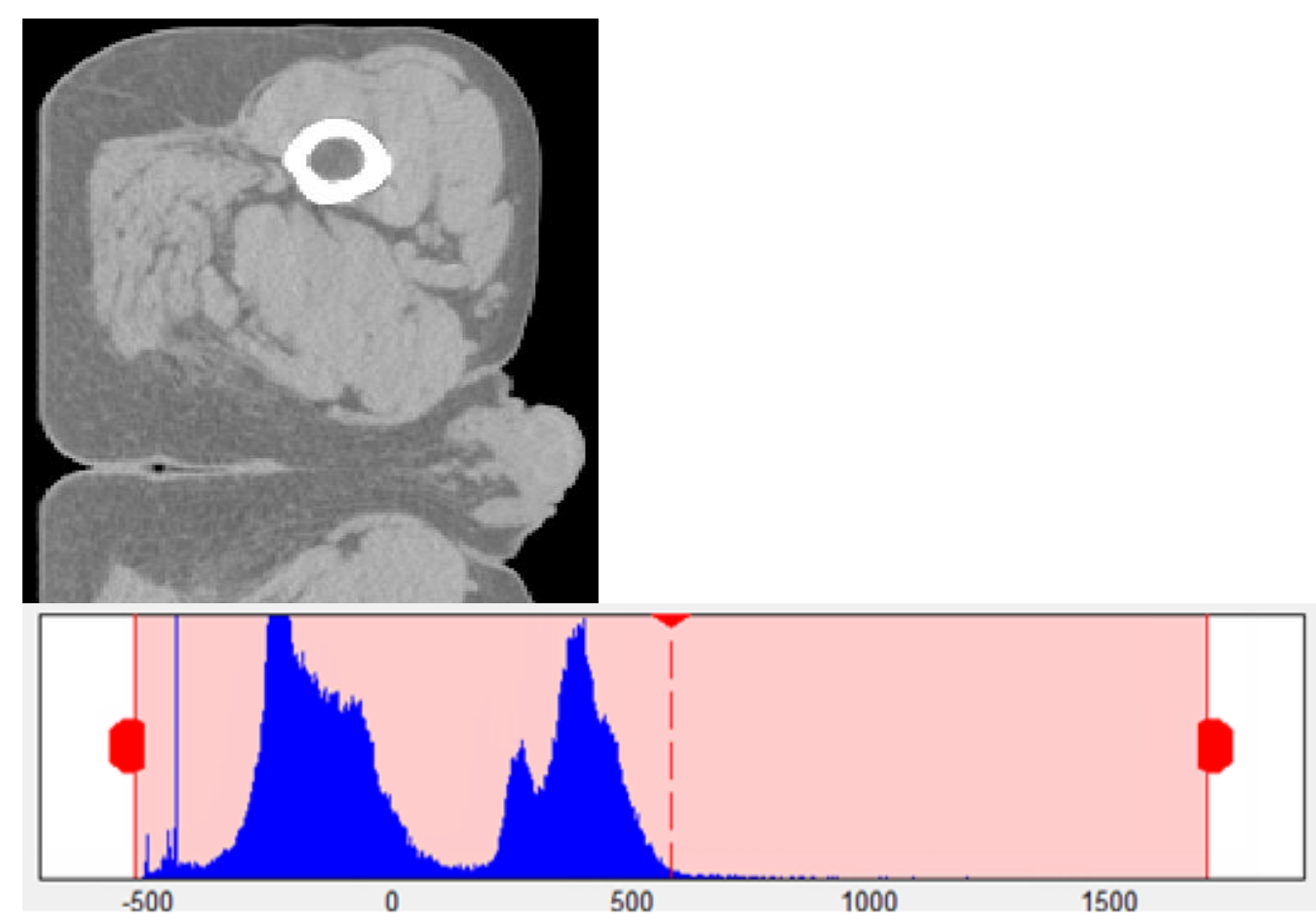

(4)

Figure 6.7. Displaying the fusion result and Contrast map of fusing two Cone Beam CTs (CBCT) 4. High resolution image with CNR of 20.0067

Intuitively the details of source images are transferred to a composite image through the proposed method. The fatty portions and details are better in composite image than the source images. Quantitative CNR is also better for the integrated image. Visually comparing the contrast map of all the provided images in Fig 6.7., contrast map of proposed method is almost similar to that of high dose image apart from some minor variants in the gray level range of 0 to 250.

All the experiments are performed in the environment of Pentium dual core CPU $2.93 \mathrm{GHz}$ with 2 GB RAM PC operating with Matlab 13. 


\section{Chapter 7. Conclusion and Future Works}

\subsection{Image fusion with simultaneous controlled orthogonal matching pursuit}

Medical Image fusion plays a vital role in clinical diagnosis. We proposed a simultaneous sparse fusion method where the images are integrated in sparse domain. Sparse coding and fusion process is done parallelly by simultaneous controlled orthogonal matching pursuit (SCStOMP) utilizing MDCT dictionary. Experiments are done in low dose and medium dose images to check the performance of the proposed method and results are compared with the conventional sparse fusion methods and SOMP fusion method. Based on the qualitative and quantitative analysis, the proposed method produces superior results than SOMP fusion method and OMP fusion method. Use of SCSt-OMP methods increases the computational efficiency since each stage allows multiple atoms but sliding window technique makes the process slightly time consuming. Even though the fusion results are superior, this fusion assumes the source images to be noiseless. But source images are prone to noise and for noisy images, the fused result will have reduced PSNR. Tuning the error stopping criterion with respect to the noise intensity would be the ideal idea for further improvement. 


\subsection{Image fusion with Joint Sparse Fusion}

Another method based on Joint Sparse Representation (JSR) is aimed to effectively integrate multi-dosage images to enhance the details necessary for diagnosis. This framework makes use of the proposed SCSt-OMP method for simultaneous sparse coding in JSR. And PCA fusion is used to integrate the innovative component sparse vector. It's necessary to note that many fusion algorithms are developed with the assumption that the source images are noise free. Since our algorithm is experimented on multi-dosage images, low dose CT images are prone to noise. Unlike traditional fusion methods, the proposed Joint Sparse fusion method simultaneously fuses and denoises the image by tuning the error threshold with respect to the noise intensity. Visually and quantitatively, the experimental results show that the proposed method has effectively expressed the geometric structures and edges and has proved to outperform some of the state-ofthe-art fusion algorithms. Objective results and contrast map shows that combination of PCA fusion and weighted average fusion works great for fusing multi-dosage images than the other combinations.

\subsection{Fusion based on Focused Vector}

In this paper a novel sparse image fusion method is proposed for fusing low dose CT images based on focused vector. This method is the extension of the proposed Joint Sparse PCA fusion method where fusion result is constructed from initial fused image and focused vector. In this method, sparse coding stage utilizes a dictionary trained from high resolution CT images and denoises the low dose image by fusing low dose and medium dose CT images in sparse domain. The results of the proposed method outperform all the other existing fusion methods presented here in terms of qualitative and quantitative measurements when tested on both noisy and noiseless images. Contrast map the fusion result of this proposed method is almost same as high dose image. This framework effectively fuses the information without the introduction of artifacts, transfers the details from source images to composite image, suppresses noise without losing any detail. The dictionary used here is trained off-line. This work can be further refined exploiting dictionaries that are trained so that they will be suitable for different source images. 


\section{References}

[1] S.Mallat, “A wavelet tour of signal processing”, Academic Press, 1999.

[2] EricWeisstein.MathWorld[Online]: http://mathworld.wolfram.com/MoorePenroseMatrixInverse.html

[3] T. Strohmer, and R. Heath Jr, "Grassmannian frames with applications to coding and communications”, Appl. Comp. Harm. Anal., vol.14(3): 257--275, 2003.

[4] David L. Donoho, Michael Elad ,"Optimally sparse representation in general (nonorthogonal) dictionaries via $\ell_{1}$ minimization", Proceedings of the National Academy of Sciences, Vol. 100, No. 5. (04 March 2003), pp. 2197-2202.

[5] Tibshirani. R, "Regression shrinkage and selection via the lasso.”, Vol. 58, No. 1, pp. 267-288., 1996

[6] Simon Perkins, James Theiler, "Online Feature Selection Using Grafting “, International Conference on Machine Learning 2013.

[7] Jinseog Kim, Yuwon Kim and Yongdai Kim," A gradient-based optimization algorithm for lasso", Journal of Computational and Graphical Statistics Volume 17, Issue 4, 2008.

[8] Tropp, J.A., "Greed is good: algorithmic results for sparse approximation", Information Theory, IEEE Transactions on (Volume:50, Issue: 10 , Page: 2231 - 2242), Oct. 2004.

[9] Mallat, S.G. ,"Matching pursuits with time-frequency dictionaries", Signal Processing, IEEE Transactions on (Volume:41, Issue:12, Page: 3397 - 3415 ), Dec. 1993.

[10] S. Chen, S.A. Billings and W. Luo, "Orthogonal least squares methods and their application to nonlinear system identification," International Journal of Control, vol. 50, no. 5, pp. 1873-1896, 1989.

[11] Y.C. Pati, R. Rezaiifar and P.S. Krishnaprasad, "Orthogonal matching pursuit: recursive function approximation with applications to wavelet decomposition," Conference Record of The Twenty Seventh Asilomar Conference on Signals, Systems and Computers, 1993.

[12] David L. Donoho, Yaakov Tsaig, Iddo Drori , Jean-luc Starck, "Stage wise Orthogonal Matching Pursuit”, IEEE Transactions on Information Theory, January 2012; 58:1094-1121

[13] Ahmed, N, Natarajan, T, Rao K. R, "Discrete Cosine Transform", IEEE Transactions on Computers C-23 (1), pp. 90-93, January 1974.

[14] M. N. Do and M. Vetterli, "The contourlet transform: an efficient directional multiresolution image representation", IEEE Transactions on Image Processing, vol. 14, no. 12, pp. 2091-2106, Dec. 2005.

[15] Rubinstein, R., "Dictionaries for Sparse Representation Modeling", Proceedings of the IEEE (Volume:98, Issue: 6 ,pp. 1045 - 1057), June 2010.

[16] Michal Aharon, Michael Elad, and Alfred Bruckstein (2006), "K-SVD: An Algorithm for Designing Overcomplete Dictionaries for Sparse Representation", pp. 4311 - 4322. 
[17] S. Mallat, “A Wavelet Tour of Signal Processing”, 2nd ed. San Diego, CA: Academic, 1999.

[18] N. G. Kingsbury, "Complex wavelets for shift invariant analysis and filtering of signals," Applied and Computational Harmonic Analysis, no. 10, pp. 234-252, 2001.

[19] Olshausen BA, and Field DJ. (1996). "Emergence of Simple-Cell Receptive Field Properties by Learning a Sparse Code for Natural Images." Nature, 381: 607-609.

[20] K. Engan, S. O. Aase, J. H. Husoy, "Method of Optimal Directions for Frame Design," in Proc. ICASSP'99, Phoenix, USA, pp. 2443--2446, March, 1999.

[21] K. Engan, B. Rao, and K. Kreutz-Delgado, "Frame design using FOCUSS with method of optimized directions (MOD)”, in Proc. NORSIG ‘99, Oslo, Norway, Sept. 1999, pp. 65--69.

[22] Ren Ng, Ravi Ramamoorthi, Pat Hanrahan, "Triple product wavelet integrals for all-frequency relighting”, ACM Transactions on Graphics (TOG) - Proceedings of ACM SIGGRAPH 2004, Volume 23 Issue 3, August 2004 Pages 477-487.

[23] Chui, Charles K. (1992). “An Introduction to Wavelets.” San Diego: Academic Press.

[24] Michael Elad, "Sparse and Redundant Representations: From Theory to Applications in Signal and Image Processing", Springer, August 2010.

[25] Ishita Dea, Bhabatosh Chandab, "A simple and efficient algorithm for multifocus image fusion using morphological wavelets", Journal Signal Processing archive Volume 86 Issue 5,Pages 924-936, May 2006.

[26] A. Goshtasby and C. Page, "A Multiple Image Segmentation Technique with Subpixel Accuracy," Proc. Computer Vision and Pattern Recognition, 1983, pp. 157-158.

[27] A. Goshtasby, "Registration of Images with Geometric Distortions," IEEE Trans. Geoscience and Remote Sensing, vol. 26, no. 1, 1988, pp. 60-64.

[28] L. Kitchen, A. Rosenfeld, "Gray-level corner detection" Pattern Recognition Lett. , 1 : 2 (1982) pp. 95-102.

[29] L. Zagorchev and A. Goshtasby, "A comparative study of transformation functions for nonrigid image registration," IEEE Trans. Image Processing, vol. 15, no. 3, 2006, 529-538.

[30] Deubel, H. and Schneider, W.X.(1996),"Saccade target selection and object recognition: evidence for a common attentional mechanism.", Vision Res., 36: 1827-1837.

[31] Ardeshir Goshtasby, A; Nikolov, SG,'Image fusion: Advances in the state of the art.", Information Fusion: Special Issue on Image Fusion: Advances in the State of the Art, Vol. 8 (2), 04.2007, pp. 114 118.

[32] Gemma Piella, "A general framework for multiresolution image fusion: from pixels to regions.", Information Fusion 4 (4), pp. 259-280, 586, 2003.

[33] R. A. Brooks and G. DiChiro, "Principles of computer assisted tomography (CAT) in radiographic and radioisotopic imaging," Phys. Med. Biol., vol. 21, pp. 689- 732, 1976. 
[34] Tinsu Pan, "Computed Tomography: from Photon Statistics to Modern Cone-Beam CT", The Journal of Nuclear Medicine, 2009;50:1194.

[35] Cormack AM," Early two-dimensional reconstruction and recent topics stemming from it.", Med Phys 7(4):277. Nobel Lecture, 8 December, 1979.

[36] Hounsfield, GN. 1973. "Computerized transverse axial scanning (tomography): Part I", Brit J Radiol 46:1016-1022.

[37] Kak A. and Slaney Malcolm,"Principles of Computed Tomography Imaging”, 1988.

[38] Mikael Sandborg ,"Computed Tomography: Physical principles and biohazards ”, Report / Department of Radiology, Linköping University, 1990-1997, ISSN 1102-1799; 81.

[39] Jones DG, Shrimpton P C," Survey of CT practice in the UK Part 3: Normalised organ doses calculated using Monte Carlo techniques", National Radio logical Protection Board, Chilton, United Kingdom 1991; NRPB-R250.

[40] ICRP, International Commission on Radiological Protection. 1990 Recommendations of the International Commission on Radiological Protection. 1991; Annals of the ICRP, Publication 60, Oxford: Pergamon.

[41] National Council on Radiation Protection \& Measurements, "Report No. 160 - Ionizing Radiation Exposure of the Population of the United States (2009)".

[42] Borsdorf, A., FriedrichAlexander, Raupach, R. , Hornegger, J. "'Separate CT-reconstruction for 3D wavelet based noise reduction using correlation analysis", Nuclear Science Symposium Conference Record, 2007. NSS '07. IEEE,P: 2633 - 2638.

[43] Hu H, He HD, Foley WD, Fox SH,' Four multidetector-row helical CT: image qualityand volume coverage speed", Radiology 2000; pp.55-62.

[44] http://www.iambiomed.com/equipments/ct.php

[45] A. Saleem, A. Beghdadi and B. Boashash," Image fusion-based contrast enhancement", Journal on Image and Video Processing, 2012, pp.1-17.

[46] Y. Zhou, "Principle Component Analysis Based image Fusion Routine with Application to Stamping Split Detection", Ph.D. dissertation, Dept. of Automotive Engineering, University of Clemson, August 2010, pp. 76-91.

[47] M. R.Metwalli, A.H. Nasr, O. S. Farag Allah, and S. El-Rabaie, "Image fusion based on Principal Component Analysis and High-pass Filter", Presented at IEEE/ ICCES 2009 international Conference, Dec. 14-16,2009, pp. 63 - 70.

[48] H. Yin, S. Li,” Multimodal image fusion with joint sparsity model”, Opt Eng, 2011.

[49]B. Yang and S. Li,Multifocus Image Fusion and Restoration With Sparse Representation, IEEE transactions on Instrumentation and Measurement, Vol. 59, No. 4, April 2010, pp. 884-892.

[50] http://bigwww.epfl.ch/sage/soft/snr/. 
[51] Junli Tao, Shutao Li, Bin Yang, "Multimodal Image Fusion Algorithm Using Dual-Tree Complex Wavelet Transform and Particle Swarm Optimization", 6th International Conference on Intelligent Computing, ICIC 2010, Changsha, China, August 18-21, 2010.

[52] Bin Yang, Shutao Li ,"Pixel-level image fusion with simultaneous orthogonal matching pursuit", Information Fusion Volume 13, Issue 1, January 2012, pp.10-19.

[53] Hui Li, Manjunath, Mitra.S.K,'Multi-sensor image fusion using the wavelet transform", Image Processing, 1994. Proceedings. ICIP-94., IEEE International Conference Volume:1, pp: 51 - 55.

[54] Xydeas, C.S., Petrovic. V.,“ Objective image fusion performance measure”, Electronics Letters (Volume:36, Issue: 4 ),pp:308-309.

[55] Zhou Wang, Bovik, A.C., Sheikh, H.R., Simoncelli.E.P.,'Image Quality Assessment: From Error Visibility to Structural Similarity", Image Processing, IEEE Transactions on Volume:13, Issue:4 , pp:600-612.

[56] CJ Martin, "The importance of radiation quality for optimisation in radiology", Biomedical Imaging and Intervention Journal, 2007, pp. e38.

[57] S.G. Nikolov., P.R. Hill., D.R. Bull., C.N. Canagarajah., "Wavelets for image fusion", A. Petrosian, F. Meyer (Eds.), Wavelets in Signal and Image Analysis, Computational Imaging and Vision Series, Kluwer Academic Publishers, Dordrecht, The Netherlands (2001). pp. 213-244.

[58] P. Burt, R. Kolczynski,"Enhanced image capture through fusion", Proceedings of the 4th International Conference on Computer Vision, 1993, pp. 173-182.

[59] L. Rebollo-Neira and D. Lowe, "Optimized orthogonal matching pursuit approach,"IEEE Signal Processing Letters, pp.137-140, 2002.

[60] Qiheng Zhang ; Yuli Fu ; Haifeng Li ; Jian Zou "Dictionary learning method for joint sparse representation-based image fusion”, Opt. Eng. 52(5), 057006 (May 22, 2013), pp. 1-11.

[61] Aline Bonami,Bruno Demange, and Philippe Jaming, "Hermite functions and uncertainty principles for the Fourier and the windowed Fourier transforms", Volume 19,2003, pp. 23-55.

[62] M. F. Duarte, S. Sarvotham, D. Baron, M. B. Wakin, and R. G. Baraniuk, "Distributed Compressed Sensing of Jointly Sparse Signals", Proceedings of the 39th Asilomar Conference on Signals, Systems and Computation, 2005, pp.1537-1541. 


\section{List of Publications}

1. Anuyogam Venkataraman, Javad Alirezaie, Paul Babyn and Alireza Ahmadian, "Medical Image Fusion Based on Joint Sparse Method”, 2nd Middle East Conference on Biomedical Engineering, February 2014, pp. 103-106.

2. Anuyogam Venkataraman, Javad Alirezaie, Paul Babyn and Alireza Ahmadian, "Multi dose Computed Tomography Image Fusion Based on Hybrid Sparse Methodology”, accepted in 36th Annual International IEEE EMBS Conference 2014 to, Chicago, Illinois, USA. 